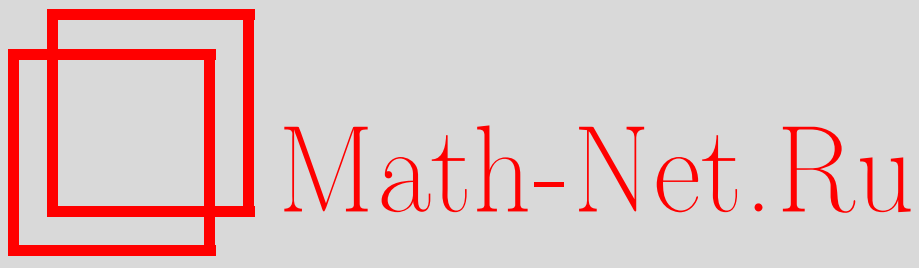

И. К. Лифанов, Л. Н. Полтавский, Пространства дробных отношений, дискретные операторы и их приложения. II, Матем. сб., 1999, том 190, номер 11, 67-134

DOI: https://doi.org/10.4213/sm440

Использование Общероссийского математического портала Math-Net.Ru подразумевает, что вы прочитали и согласны с пользовательским соглашением http://www . mathnet.ru/rus/agreement

Параметры загрузки:

IP : 54.224 .135 .184

26 апреля 2023 г., $14: 31: 36$ 


\title{
Пространства дробных отношений, дискретные операторы и их приложения. II
}

\begin{abstract}
В работе строится теория дискретных операторов в пространствах дробных отношений. Получена теорема об устойчивости дискретных операторов при гладких возмущениях. На основе этого с помощью специалњных квадратурњых формул типа прямоугольников доказана сходимость приближенных решений гиперсингулярных интегральных уравнений к точньм и дано математическое обоснование метода дискретных замкнутых вихревых рамок. Полученная схема рассуждений применяется также к исследованию разностных уравнений при решении однородной задачи Дирихле для общего эллиптического уравнения с переменньми коэффициентами второго порядка.
\end{abstract}

Библиография: 7 названий.

\section{Введение}

В настояшее время широкое применение получил метод дискретных замкнутых вихревых рамок численного решения пространственных задач аэродинамики и аналогичный метод численного решения пространственных задач дифракции волн [1]. Этот метод является методом численного решения соответствующих гиперсингулярных интегральных уравнений с помощью специальных квадратурных сумм типа прямоугольников для гиперсингулярных интегралов. Для доказательства сходимости численных решений, полученных методом дискретных замкнутых вихревых рамок, к точным в работе [2] авторов была построена теория пространств дробных отношений.

В предлагаемой работе строится теория дискретных операторов в пространствах дробных отношений. Получена теорема об устойчивости дискретных операторов при гладких возмущениях. На основе этого доказана сходимость приближенных решений гиперсингулярных интегральных уравнений к точным и дано математическое обоснование метода дискретных замкнутых вихревых рамок. Полученная схема рассуждений применяется также к исследованию разностных уравнений при решении однородной задачи Дирихле для обшего эллиптического уравнения с переменными коэффициентами второго порядка.

\section{§1. Аппроксимация операторов}

ОПРЕДЕЛЕНИЕ 1.1. Семейство операторов $\mathrm{T}_{h}$, отображающих пространства $M(r, h)$ в $M(q, h)$, называется ограниченным, если $\left\|\mathrm{T}_{h}\right\| \leqslant C$, где $C$ не зависит от $h$. 
ОПРЕДЕЛЕНИЕ 1.2. Будем говорить, что ограниченное семейство операторов $\mathrm{T}_{h}$, отображающих $M(r, h), r>0$, в $M(q, h), q<0$, cлабо aпnроксимирует ограниченный оператор $\mathrm{T}: H_{r}\left(\mathbb{R}_{n}\right) \rightarrow H_{q}\left(\mathbb{R}_{n}\right)$, если $\mathrm{T}_{h} \prod_{h}^{*} a(x) \rightarrow \mathrm{T} a(x)$ по норме $H_{q}\left(\mathbb{R}_{n}\right)$ при $|h| \rightarrow 0 \forall a(x) \in C_{0}^{\infty}\left(\mathbb{R}_{n}\right)$ (определение $\prod_{h}^{*}$ см. в [2]).

ПРЕДЛОЖЕНИЕ 1.1. Предположим, что ограниченное семейство операторов $\mathrm{T}_{h}: M(r, h) \rightarrow M(q, h), r>0, q<0$, слабо аппроксимирует ограниченный оператор $\mathrm{T}: H_{r}\left(\mathbb{R}_{n}\right) \rightarrow H_{q}\left(\mathbb{R}_{n}\right)$. Тогда для любой $u(x) \in H_{r}\left(\mathbb{R}_{n}\right)$ имеет место соотношение

$$
\lim _{|h| \rightarrow 0}\left\|\mathrm{~T} u(x)-\mathrm{T}_{h} \prod_{h} u(x)\right\|_{H_{q}\left(\mathbb{R}_{n}\right)}=0
$$

где $\prod_{h}-$ интегральный проектор (см. [2; определение 5.1]).

ДокаЗАТЕЛЬСтво. По теореме 5.1 из [2] $\prod_{h} u(x) \in M(r, h)$, следовательно, $\mathrm{T}_{h} \prod_{h} u(x) \in M(q, h)$, а из теоремы 2.1 (см. [2]) следует, что $\mathrm{T}_{h} \prod_{h} u(x) \in$ $H_{q}\left(\mathbb{R}_{n}\right)$. Поэтому левая часть (1.1) имеет смысл. Пусть $\varphi(x) \in C_{0}^{\infty}\left(\mathbb{R}_{n}\right)$, тогда, используя предложение 5.9 (см. [2]), а также неравенство

$$
\begin{aligned}
\left\|\mathrm{T}_{h} \prod_{h} \varphi(x)-\mathrm{T} \varphi(x)\right\|_{H_{q}\left(\mathbb{R}_{n}\right)} \leqslant & \left\|\mathrm{T}_{h} \prod_{h}^{*} \varphi(x)-\mathrm{T} \varphi(x)\right\|_{H_{q}\left(\mathbb{R}_{n}\right)} \\
& +\left\|\mathrm{T}_{h}\left[\prod_{h} \varphi(x)-\prod_{h}^{*} \varphi(x)\right]\right\|_{H_{q}\left(\mathbb{R}_{n}\right)}
\end{aligned}
$$

получаем

$$
\lim _{|h| \rightarrow 0}\left\|\mathrm{~T}_{h} \prod_{h} \varphi(x)-\mathrm{T} \varphi(x)\right\|_{H_{q}\left(\mathbb{R}_{n}\right)}=0
$$

Пусть $u(x) \in H_{r}\left(\mathbb{R}_{n}\right)$ - некоторая функция. Возьмем произвольное $\varepsilon>0$, тогда, так как пространство $C_{0}^{\infty}\left(\mathbb{R}_{n}\right)$ всюду плотно в $H_{r}\left(\mathbb{R}_{n}\right)$, найдется функция $\varphi_{\varepsilon}(x) \in C_{0}^{\infty}\left(\mathbb{R}_{n}\right)$ такая, что $\left\|u(x)-\varphi_{\varepsilon}(x)\right\|_{H_{q}\left(\mathbb{R}_{n}\right)}<\varepsilon$. Из соотношения $(1.2)$ вытекает, что сушествует $\delta\left(\varepsilon, \varphi_{\varepsilon}(x)\right)>0$ такое, что для всех $h,|h|<\delta\left(\varepsilon, \varphi_{\varepsilon}(x)\right)$, справедлива оценка $\left\|\mathrm{T}_{h} \prod_{h} \varphi_{\varepsilon}(x)-\mathrm{T} \varphi_{\varepsilon}(x)\right\|_{H_{q}\left(\mathbb{R}_{n}\right)}<\varepsilon$. Используя теорему 5.1 (см. [2]) об ограниченности интегрального проектора, получаем

$$
\begin{aligned}
\| \mathrm{T}_{h} & \prod_{h} u(x)-\mathrm{T} u(x) \|_{H_{q}\left(\mathbb{R}_{n}\right)} \\
= & \left\|\mathrm{T}_{h} \prod_{h}\left(u(x)-\varphi_{\varepsilon}(x)\right)+\mathrm{T}_{h} \prod_{h} \varphi_{\varepsilon}(x)-\mathrm{T} \varphi_{\varepsilon}(x)+\mathrm{T}\left(\varphi_{\varepsilon}(x)-u(x)\right)\right\|_{H_{q}\left(\mathbb{R}_{n}\right)} \\
\leqslant & \left\|\mathrm{T}_{h}\right\| \cdot\left\|\prod_{h}\left(u(x)-\varphi_{\varepsilon}(x)\right)\right\|_{M(r, h)}+\left\|\mathrm{T}_{h} \prod_{h} \varphi_{\varepsilon}(x)-\mathrm{T} \varphi_{\varepsilon}(x)\right\|_{H_{q}\left(\mathbb{R}_{n}\right)} \\
& \quad+\|\mathrm{T}\| \cdot\left\|\varphi_{\varepsilon}(x)-u(x)\right\|_{H_{q}\left(\mathbb{R}_{n}\right)} \leqslant C_{1} \varepsilon+\varepsilon+C_{2} \varepsilon,
\end{aligned}
$$

где $C_{1}$ и $C_{2}$ не зависят от $h$. Из последнего неравенства следует предложение 1.1. 
ПРЕДЛОЖЕНИЕ 1.2. Предположсим, что ограниченное семейство операторов $\mathrm{T}_{h}: M(r, h) \rightarrow M(q, h), r>0, q<0$, слабо аппроксимирует ограниченныи оператор $\mathrm{T}: H_{r}\left(\mathbb{R}_{n}\right) \rightarrow H_{q}\left(\mathbb{R}_{n}\right)$. Тогда для любой $u(x) \in \stackrel{\circ}{H_{r}}(\Omega)$, әде $\Omega$ нормальная область, допускающая разбиение (см. [2]), имеет место соотношение

$$
\lim _{|h| \rightarrow 0}\left\|\mathrm{~T}_{h} \prod_{1 h} u(x)-\mathrm{T} u(x)\right\|_{H_{q}\left(\mathbb{R}_{n}\right)}=0
$$

где оператор $\prod_{1 h}$ является сужением интегрального проектора $\prod_{h}$ на область $\Omega_{1 h}$.

ДокАЗАтЕльство. Пусть $u(x) \in \stackrel{\circ}{H}_{r}(\Omega)$ - некоторая функция. Возьмем произвольное $\varepsilon>0$; тогда, так как пространство $C_{0}^{\infty}(\Omega)$ всюду плотно в $\stackrel{\circ}{H}_{r}(\Omega)$, найдется функция $\varphi_{\varepsilon}(x) \in C_{0}^{\infty}(\Omega)$ такая, что будет выполняться неравенство $\left\|u(x)-\varphi_{\varepsilon}(x)\right\|_{\stackrel{\circ}{H}_{r}(\Omega)}<\varepsilon$. Ввиду того что $\overline{\operatorname{supp} \varphi_{\varepsilon}(x)} \subset \Omega$, найдется $\delta>0$ такое, что $\Omega_{1 h} \supset \operatorname{supp} \varphi_{\varepsilon}(x)$ при $|h|<\delta$ и, следовательно, $\prod_{h} \varphi_{\varepsilon}(x)=\prod_{1 h} \varphi_{\varepsilon}(x)$ для $|h|<\delta$. Из предложения 1.1 следует, что существует $\delta_{1}\left(\varepsilon, \varphi_{\varepsilon}(x)\right)$ такое, что $\left\|\mathrm{T}_{h} \prod_{1 h} \varphi_{\varepsilon}(x)-\mathrm{T} \varphi_{\varepsilon}(x)\right\|_{H_{q}\left(\mathbb{R}_{n}\right)}<\varepsilon$ при $|h|<\delta_{1}\left(\varepsilon, \varphi_{\varepsilon}(x)\right)$. Отсюда, взяв $\delta_{2}=\min \left(\delta, \delta_{1}\left(\varepsilon, \varphi_{\varepsilon}(x)\right)\right)$, получим, что при $|h|<\delta_{2}$ выполняется неравенство $\left\|\mathrm{T}_{h} \prod_{1 h} \varphi_{\varepsilon}(x)-\mathrm{T} \varphi_{\varepsilon}(x)\right\|_{H_{q}\left(\mathbb{R}_{n}\right)}<\varepsilon$. Так как область $\Omega$ допускает разбиение, то оператор $\prod_{1 h}$ является равномерно ограниченным по $h=\left(h_{1}, \ldots, h_{n}\right)$ из $\stackrel{\circ}{H}_{r}(\Omega)$ в $\stackrel{\circ}{M}\left(r, h, \Omega_{1 h}\right)$. Используя этот факт, получаем при $|h|<\delta_{2}$ :

$$
\begin{aligned}
\left\|\mathrm{T}_{h} \prod_{1 h} u(x)-\mathrm{T} u(x)\right\|_{H_{q}\left(\mathbb{R}_{n}\right)} & \\
= & \left\|\mathrm{T}_{h} \prod_{1 h}\left(u(x)-\varphi_{\varepsilon}(x)\right)+\mathrm{T}_{h} \prod_{1 h} \varphi_{\varepsilon}(x)-\mathrm{T} \varphi_{\varepsilon}(x)+\mathrm{T}\left(\varphi_{\varepsilon}(x)-u(x)\right)\right\|_{H_{q}\left(\mathbb{R}_{n}\right)} \\
\leqslant & \left\|\mathrm{T}_{h}\right\| \cdot\left\|\prod_{1 h}\right\| \cdot\left\|u(x)-\varphi_{\varepsilon}(x)\right\|_{\stackrel{\circ}{H}_{r}(\Omega)}+\left\|\mathrm{T}_{h} \prod_{1 h} \varphi_{\varepsilon}(x)-\mathrm{T} \varphi_{\varepsilon}(x)\right\|_{H_{q}\left(\mathbb{R}_{n}\right)} \\
& +\|\mathrm{T}\| \cdot\left\|\varphi_{\varepsilon}(x)-u(x)\right\|_{\stackrel{\circ}{r}_{r}(\Omega)}^{<}<C_{1} \varepsilon+\varepsilon+C_{2} \varepsilon
\end{aligned}
$$

где $C_{1}$ и $C_{2}$ не зависят от $h$. Из последнего неравенства следует предложение 1.2.

ОПРЕДЕЛЕНИЕ 1.3. Ограниченный оператор Т: $H_{r}\left(\mathbb{R}_{n}\right) \rightarrow H_{q}\left(\mathbb{R}_{n}\right), r>0$, $q<0$, будем называть оператором конечного порядка, если Т $\varphi(x) \in L_{2}\left(\mathbb{R}_{n}\right)$ $\forall \varphi(x) \in C_{0}^{\infty}\left(\mathbb{R}_{n}\right)$.

ОПРЕДЕЛЕНИЕ 1.4. Будем говорить, что ограниченное семейство линейных операторов $\mathrm{T}_{h}: M(r, h) \rightarrow M(q, h), r>0, q<0$, cильно аппроксимирует ограниченный оператор $\mathrm{T}: H_{r}\left(\mathbb{R}_{n}\right) \rightarrow H_{q}\left(\mathbb{R}_{n}\right)$ конечного порядка, если $\forall \varphi(x) \in C_{0}^{\infty}\left(\mathbb{R}_{n}\right)$ вьполняется соотношение

$$
\lim _{|h| \rightarrow 0}\left\|\mathrm{~T}_{h} \prod_{h} \varphi_{\varepsilon}(x)-\mathrm{T} \varphi_{\varepsilon}(x)\right\|_{L_{2}\left(\mathbb{R}_{n}\right)}=0
$$


ОПРЕДЕЛЕНИЕ 1.5. Будем говорить, что ограниченное семейство операторов $\mathrm{T}_{h}: M(r, h) \rightarrow M(q, h), r>0, q<0$, аппроксимирует в области $\Omega$ ограниченный оператор $\mathrm{T}: H_{r}\left(\mathbb{R}_{n}\right) \rightarrow H_{q}\left(\mathbb{R}_{n}\right)$ конечного порядка, если $\forall \varphi(x) \in C_{0}^{\infty}\left(\mathbb{R}_{n}\right)$ вьполняется соотношение

$$
\lim _{|h| \rightarrow 0}\left\|\mathrm{P}_{h} \mathrm{~T}_{h} \prod_{h} \varphi(x)-\mathrm{P}_{h} \mathrm{~T} \varphi(x)\right\|_{L_{2}\left(\Omega_{1 h}\right)}=0,
$$

где $\mathrm{P}_{h}$ - оператор сужения на область $\Omega_{1 h}$.

ПРЕДЛОЖЕНИЕ 1.3. Предположим, что ограниченное семейство операторов $\mathrm{T}_{h}: M(r, h) \rightarrow M(q, h), r>0, q<0$, аппроксимирует в ограниченной области $\Omega$ ограниченный оператор $\mathrm{T}: H_{r}\left(\mathbb{R}_{n}\right) \rightarrow H_{q}\left(\mathbb{R}_{n}\right)$ конечного порядка. Тогда для любой $\varphi(x) \in C_{0}^{\infty}(\Omega)$ существует продолжение $l_{h}$ функции $\mathrm{P}_{h} \mathrm{~T}_{h} \prod_{1 h} \varphi(x)$ с области $\Omega_{1 h}$ на все $\mathbb{R}_{n}$, которое удовлетворяет условиям:

1) $l_{h} \mathrm{P}_{h} \mathrm{~T}_{h} \prod_{1 h} \varphi(x)$ является ступенчатой функичей с ячейками постоянства $D(k, h)$ и а $a(x, h)=l_{h} \mathrm{P}_{h} \mathrm{~T}_{h} \prod_{1 h} \varphi(x)=0$ nри

$$
x \in D(k, h) \subset \Omega_{2 h} \backslash \bar{\Omega}_{1 h} ;
$$

2) $\lim _{|h| \rightarrow 0}\left\|l_{h} \mathrm{P}_{h} \mathrm{~T}_{h} \prod_{1 h} \varphi(x)-\mathrm{T} \varphi(x)\right\|_{L_{2}\left(\mathbb{R}_{n}\right)}=0$.

ДокАЗАТЕЛЬСтво. Возьмем последовательность $\left\{\varepsilon_{j}>0\right\}_{j=1}^{\infty}, \varepsilon_{j} \rightarrow 0$, и произвольную функцию $\varphi_{0}(x) \in C_{0}^{\infty}(\Omega)$. Так как $\mathrm{T} \varphi(x) \in L_{2}\left(\mathbb{R}_{n}\right) \forall \varphi(x) \in C_{0}^{\infty}\left(\mathbb{R}_{n}\right)$, а множество функций из $C_{0}^{\infty}\left(\mathbb{R}_{n}\right)$ всюду плотно в $L_{2}\left(\mathbb{R}_{n}\right)$, то найдется последовательность $\left\{\varphi_{j}(x)\right\}_{j=1}^{\infty}, \varphi_{j}(x) \in C_{0}^{\infty}\left(\mathbb{R}_{n}\right)$, такая, что будет выполняться неравенство $\left\|\mathrm{T} \varphi_{0}(x)-\varphi_{j}(x)\right\|_{L_{2}\left(\mathbb{R}_{n}\right)}<\varepsilon_{j}$. По последовательности $\left\{\varepsilon_{j}\right\}_{j=1}^{\infty}$ найдем такую последовательность $\left\{\delta_{j}>0\right\}_{j=1}^{\infty}, \delta_{j} \rightarrow 0$, что для всех $h,|h|<\delta_{j}$, и для каждой фиксированной $\varphi_{j}(x)$ будет выполняться неравенство

$$
\left\|\prod_{h}^{*} \varphi_{j}(x)-\varphi_{j}(x)\right\|_{L_{2}\left(\mathbb{R}_{n}\right)}<\varepsilon_{j}
$$

Возьмем какое-нибудь $h$, удовлетворяюшее условию $\delta_{j+1} \leqslant|h|<\delta_{j}$, тогда продолжение $l_{h} \mathrm{P}_{h} \mathrm{~T}_{h} \prod_{1 h} \varphi_{0}(x)$ определим следуюшим образом:

1. $l_{h} \mathrm{P}_{h} \mathrm{~T}_{h} \prod_{1 h} \varphi_{0}(x)=\mathrm{P}_{h} \mathrm{~T}_{h} \prod_{1 h} \varphi_{0}(x)$, если $x \in D(k, h) \subset \Omega_{1 h}$;

2. $l_{h} \mathrm{P}_{h} \mathrm{~T}_{h} \prod_{1 h} \varphi_{0}(x)=\prod_{h}^{*} \varphi_{j}(x)$, если $x \in D(k, h) \subseteq \mathbb{R}_{n} \backslash \bar{\Omega}_{2 h}$;

3. $l_{h} \mathrm{P}_{h} \mathrm{~T}_{h} \prod_{1 h} \varphi_{0}(x)=0$, если $x \in D(k, h) \subset \Omega_{2 h} \backslash \bar{\Omega}_{1 h}$.

Из конструкции $l_{h}$ ясно, что выполняется свойство 1 ), указанное в предложении 1.3. Далее рассмотрим

$$
\begin{aligned}
\| l_{h} \mathrm{P}_{h} \mathrm{~T}_{h} & \prod_{1 h} \varphi_{0}(x)-\mathrm{T} \varphi_{0}(x) \|_{L_{2}\left(\mathbb{R}_{n}\right)}^{2} \\
= & \left\|l_{h} \mathrm{P}_{h} \mathrm{~T}_{h} \prod_{1 h} \varphi_{0}(x)-\mathrm{T} \varphi_{0}(x)\right\|_{L_{2}\left(\Omega_{1 h}\right)}^{2} \\
& \quad+\left\|l_{h} \mathrm{P}_{h} \mathrm{~T}_{h} \prod_{1 h} \varphi_{0}(x)-\mathrm{T} \varphi_{0}(x)\right\|_{L_{2}\left(\Omega_{2 h} \backslash \bar{\Omega}_{1 h}\right)}^{2} \\
& \quad+\left\|l_{h} \mathrm{P}_{h} \mathrm{~T}_{h} \prod_{1 h} \varphi_{0}(x)-\mathrm{T} \varphi_{0}(x)\right\|_{L_{2}\left(\mathbb{R}_{n} \backslash \bar{\Omega}_{2 h}\right)}^{2} \\
= & I_{1}(h)+I_{2}(h)+I_{3}(h) .
\end{aligned}
$$


Так как $\Omega_{1 h} \supset \overline{\operatorname{supp} \varphi_{0}(x)}$ при $|h| \rightarrow 0, \varphi_{0}(x) \in C_{0}^{\infty}(\Omega)$, то из условия аппроксимации получим, что

$$
\lim _{|h| \rightarrow 0} I_{1}(h)=0
$$

Так как $\mu\left(\Omega_{2 h} \backslash \bar{\Omega}_{1 h}\right) \rightarrow 0$ при $|h| \rightarrow 0\left(\right.$ где $\mu\left(\Omega_{2 h} \backslash \bar{\Omega}_{1 h}\right)$ - мера множества $\left.\Omega_{2 h} \backslash \bar{\Omega}_{1 h}\right)$, то

$$
\lim _{|h| \rightarrow 0} I_{2}(h)=0
$$

Пусть $\delta_{j+1} \leqslant|h|<\delta_{j}$, тогда из (1.7) получим:

$$
\begin{aligned}
I_{3}^{1 / 2}(h) & \leqslant\left\|l_{h} \mathrm{P}_{h} \mathrm{~T}_{h} \varphi_{0}(x)-\varphi_{j}(x)\right\|_{L_{2}\left(\mathbb{R}_{n} \backslash \bar{\Omega}_{2 h}\right)}+\left\|\varphi_{j}(x)-\mathrm{T} \varphi_{0}(x)\right\|_{L_{2}\left(\mathbb{R}_{n}\right)} \\
& \leqslant\left\|\prod_{h}^{*} \varphi_{j}(x)-\varphi_{j}(x)\right\|_{L_{2}\left(\mathbb{R}_{n}\right)}+\varepsilon_{j}<2 \varepsilon_{j} .
\end{aligned}
$$

Ввиду того что $j \rightarrow \infty, \varepsilon_{j} \rightarrow 0$ при $|h| \rightarrow 0$, из (1.11) следует

$$
\lim _{|h| \rightarrow 0} I_{3}(h)=0
$$

Из (1.9), (1.10), (1.12) следует (1.6). Из последнего замечания вытекает предложение 1.3 .

ОПРЕДЕЛЕНИЕ 1.6. Onератор сужения $\mathrm{P}_{1 h}$ ступенчатой функции $a(x, h)$ на ограниченную область $\Omega$ определим следующим образом: $\mathrm{P}_{1 h} a(x, h)=a(x, h)$ при $x \in D(k, h) \subset \Omega_{1 h} ; \mathrm{P}_{1 h} a(x, h)=0$ при $x \in \Omega \backslash \bar{\Omega}_{1 h}$.

ПРЕДЛОЖЕНИЕ 1.4. Предположим, что ограниченное семейство операторов $\mathrm{T}_{h}: M(r, h) \rightarrow M(q, h), r>0, q<0$, аппроксимирует в ограниченной, нормальной, допускающей разбиение области $\Omega$ ограниченный оператор $\mathrm{T}: H_{r}\left(\mathbb{R}_{n}\right) \rightarrow H_{q}\left(\mathbb{R}_{n}\right)$ конечного порядка. Тогда для любой $u(x) \in \stackrel{\circ}{H}_{r}(\Omega)$ имеет место равенство:

$$
\lim _{|h| \rightarrow 0}\left\|\mathrm{P}_{1 h} \mathrm{~T}_{h} \prod_{1 h} u(x)-\mathrm{PT} u(x)\right\|_{H_{q}(\Omega)}=0
$$

әде $\mathrm{PT} и(x)$ - сужение Т $и(x)$ на область $\Omega$, m.е. $\mathrm{PT}(x)$ есть сужсние линейного функционала Ти $(x)$ над $H_{-q}\left(\mathbb{R}_{n}\right)$ на подпространство $\stackrel{\circ}{H}{ }_{-q}(\Omega)$.

ДокАЗАТЕльство. Пусть $u(x) \in \stackrel{\circ}{H}_{r}(\Omega)$ - некоторая функция. Возьмем $\varepsilon>0$, тогда найдется функция $\varphi_{\varepsilon}(x) \in C_{0}^{\infty}(\Omega)$ такая, что

$$
\left\|u(x)-\varphi_{\varepsilon}(x)\right\|_{\stackrel{\circ}{H}_{r}(\Omega)}<\varepsilon
$$

Из предложения 1.3 следует, что сушествуют такие $\delta\left(\varepsilon, \varphi_{\varepsilon}(x)\right)>0$ и функция $l_{h} \mathrm{P}_{h} \mathrm{~T}_{h} \prod_{1 h} \varphi_{\varepsilon}(x)$, что при $|h|<\delta\left(\varepsilon, \varphi_{\varepsilon}(x)\right)$ будет выполняться оценка

$$
\left\|\mathrm{T} \varphi_{\varepsilon}(x)-l_{h} \mathrm{P}_{h} \mathrm{~T}_{h} \prod_{1 h} \varphi_{\varepsilon}(x)\right\|_{L_{2}\left(\mathbb{R}_{n}\right)}<\varepsilon .
$$


Далее при $|h|<\delta\left(\varepsilon, \varphi_{\varepsilon}(x)\right)$ получим:

$$
\begin{aligned}
& \left\|\mathrm{P}_{1 h} \mathrm{~T}_{h} \prod_{1 h} u(x)-\mathrm{PT} u(x)\right\|_{H_{q}(\Omega)} \\
& \leqslant\left\|\mathrm{P}_{1 h} \mathrm{~T}_{h} \prod_{1 h} u(x)-\mathrm{P}_{1 h} \mathrm{~T}_{h} \prod_{1 h} \varphi_{\varepsilon}(x)\right\|_{H_{q}(\Omega)} \\
& \quad+\left\|\mathrm{P}_{1 h} \mathrm{~T}_{h} \prod_{1 h} \varphi_{\varepsilon}(x)-\mathrm{PT} \varphi_{\varepsilon}(x)\right\|_{H_{q}(\Omega)}+\left\|\mathrm{PT} \varphi_{\varepsilon}(x)-\mathrm{PT} u(x)\right\|_{H_{q}(\Omega)} \\
& =I_{1}(h)+I_{2}(h)+I_{3}(h) .
\end{aligned}
$$

Из определения нормы в $H_{q}(\Omega)$ следует, что

$$
I_{3}(h)<\|\mathrm{T}\| \cdot \varepsilon .
$$

Так как область $\Omega$ допускает разбиение (см. [2; определение 6.6]), то $\prod_{1 h}-$ равномерно ограниченное семейство операторов из $\stackrel{\circ}{H}_{r}(\Omega)$ в $\stackrel{\circ}{M}\left(r, h, \Omega_{1 h}\right)$. Предложение 6.5 [2] позволяет заключить, что операторы $\mathrm{P}_{1 h}$ представляют собой равномерно ограниченное семейство операторов из $M\left(q, h, \Omega_{1 h}\right)$ в $H_{q}(\Omega)$. Из условий предложения 1.4 и определения нормы в $M\left(q, h, \Omega_{1 h}\right)$ (см. [2; определение 6.4]) следует, что семейство операторов $\mathrm{T}_{h}$ равномерно ограничено из $\stackrel{\circ}{M}\left(r, h, \Omega_{1 h}\right)$ в $M\left(q, h, \Omega_{1 h}\right)$. Из сделанных выше замечаний получим:

$$
\begin{aligned}
I_{1}(h) & \leqslant\left\|\mathrm{P}_{1 h}\right\| \cdot\left\|\mathrm{T}_{h} \prod_{1 h}\left(u(x)-\varphi_{\varepsilon}(x)\right)\right\|_{M\left(q, h, \Omega_{1 h}\right)} \\
& \leqslant\left\|\mathrm{P}_{1 h}\right\| \cdot\left\|\mathrm{T}_{h}\right\| \cdot\left\|\prod_{1 h}\right\| \cdot\left\|u(x)-\varphi_{\varepsilon}(x)\right\|_{H_{r}(\Omega)}<k \cdot \varepsilon,
\end{aligned}
$$

где $k$ не зависит от $h=\left(h_{1}, \ldots, h_{n}\right)$.

Перейдем к рассмотрению $I_{2}(h)$. Возьмем $l_{h} \mathrm{P}_{1 h} \mathrm{~T}_{h} \prod_{1 h} \varphi_{\varepsilon}(x)$ в качестве продолжения функции $\mathrm{P}_{1 h} \mathrm{~T}_{h} \prod_{1 h} \varphi_{\varepsilon}(x)$ как элемента $H_{q}\left(\mathbb{R}_{n}\right)$, что можно сделать ввиду свойств продолжения $l_{h}$, доказанных в предложении 1.3 ; в качестве продолжения $\mathrm{PT} \varphi_{\varepsilon}(x)$ возьмем $\mathrm{T} \varphi_{\varepsilon}(x)$. Тогда на основании определения нормы в $H_{q}(\Omega)$ и (1.15) имеем:

$$
\begin{aligned}
I_{2} & \leqslant\left\|\mathrm{~T} \varphi_{\varepsilon}(x)-l_{h} \mathrm{P}_{h} \mathrm{~T}_{h} \prod_{1 h} \varphi_{\varepsilon}(x)\right\|_{H_{q}\left(\mathbb{R}_{n}\right)} \\
& \leqslant C\left\|\mathrm{~T} \varphi_{\varepsilon}(x)-l_{h} \mathrm{P}_{h} \mathrm{~T}_{h} \prod_{1 h} \varphi_{\varepsilon}(x)\right\|_{L_{2}\left(\mathbb{R}_{n}\right)} \leqslant C \cdot \varepsilon,
\end{aligned}
$$

где $C$ не зависит от $h$.

Из (1.16)-(1.19) ввиду произвольности $\varepsilon$ следует предложение 1.4 .

ПРЕДЛОЖЕНИЕ 1.5. Предположим, что ограниченное семейство операторов $\mathrm{T}_{h}: M(r, h) \rightarrow M(q, h), r>0, q<0$, аппроксимирует в ограниченной, нормальной, допускающей разбиение области $\Omega$ ограниченный оператор $\mathrm{T}: H_{r}\left(\mathbb{R}_{n}\right) \rightarrow H_{q}\left(\mathbb{R}_{n}\right)$. Тогда для любой функиии $и(x) \in \stackrel{\circ}{H}_{r}(\Omega)$ существует продолжение $l_{h} \mathrm{P}_{h} \mathrm{~T}_{h} \prod_{1 h} u(x) \in M(q, h)$ функиии $\mathrm{P}_{h} \mathrm{~T}_{h} \prod_{1 h} u(x) \in$ $M\left(q, h, \Omega_{1 h}\right)$ maкое, что

$$
\lim _{|h| \rightarrow 0}\left\|l_{h} \mathrm{P}_{h} \mathrm{~T}_{h} \prod_{1 h} u(x)-\mathrm{T} u(x)\right\|_{H_{q}\left(\mathbb{R}_{n}\right)}=0 .
$$


ДокаЗАтЕЛЬство. Пусть $u(x) \in \stackrel{\circ}{H}_{r}(\Omega)$ - некоторая функция. Возьмем последовательность $\left\{\varepsilon_{j}>0\right\}_{j=1}^{\infty}, \varepsilon_{j} \searrow 0$; тогда, так как оператор Т ограниченный и пространство функций $C_{0}^{\infty}(\Omega)$ всюду плотно в $\stackrel{\circ}{H}_{r}(\Omega)$, найдется последовательность $\left\{\varphi_{j}(x)>0\right\}_{j=1}^{\infty}, \varphi_{j}(x) \in C_{0}^{\infty}(\Omega)$, для которой будет выполняться $\left\|\mathrm{T} u(x)-\mathrm{T} \varphi_{j}(x)\right\|_{H_{q}\left(\mathbb{R}_{n}\right)}<\varepsilon_{j}$. Из предложения 1.3 следует, что сушествуют последовательность $\left\{\delta_{j}\left(\varepsilon_{j}, \varphi_{j}(x)\right)>0\right\}_{j=1}^{\infty}, \delta_{j} \searrow 0$, и продолжения $l_{h} \mathrm{P}_{h} \mathrm{~T}_{h} \prod_{1 h} \varphi_{j}(x)$ такие, что для всех $h,|h|<\delta_{j}\left(\varepsilon_{j}, \varphi_{j}(x)\right)$, и для каждой фиксированной функции $\varphi_{j}(x)$ будет выполняться неравенство

$$
\left\|l_{h} \mathrm{P}_{h} \mathrm{~T}_{h} \varphi_{j}(x)-\mathrm{T} \varphi_{j}(x)\right\|_{H_{q}\left(\mathbb{R}_{n}\right)}<\varepsilon_{j}
$$

а также $\Omega_{1 h} \supset \operatorname{supp} \varphi_{j}(x)$.

Возьмем какое-нибудь $h$, удовлетворяюшее неравенству $\delta_{j+1}\left(\varepsilon_{j+1}, \varphi_{j+1}(x)\right) \leqslant$ $|h|<\delta_{j}\left(\varepsilon_{j}, \varphi_{j}(x)\right)$; тогда продолжение $l_{h} \mathrm{P}_{h} \mathrm{~T}_{h} \prod_{1 h} u(x)$ определим соотношением

$$
l_{h} \mathrm{P}_{h} \mathrm{~T}_{h} \prod_{1 h} u(x)=\mathrm{T}_{h} \prod_{1 h} u(x)-\mathrm{T}_{h} \prod_{1 h} \varphi_{j}(x)+l_{h} \mathrm{P}_{h} \mathrm{~T}_{h} \prod_{1 h} \varphi_{j}(x) .
$$

Из конструкции продолжения $l_{h} \mathrm{P}_{h} \mathrm{~T}_{h} \prod_{1 h} \varphi_{j}(x)$, указанной в предложении 1.3 , следует, что $\mathrm{P}_{h} l_{h} \mathrm{P}_{h} \mathrm{~T}_{h} \prod_{1 h} u(x)=\mathrm{P}_{h} \mathrm{~T}_{h} \prod_{1 h} u(x)$ и, значит, продолжение, задаваемое (1.22), корректно.

Пусть $|h|<\delta_{n_{0}}\left(\varepsilon_{n_{0}}, \varphi_{n_{0}}(x)\right)$, тогда, используя теорему 2.1 (см. [2]), получаем при $\delta_{m+1}\left(\varepsilon_{m+1}, \varphi_{m+1}(x)\right) \leqslant|h|<\delta_{m}\left(\varepsilon_{m}, \varphi_{m}(x)\right)<\delta_{n_{0}}\left(\varepsilon_{n_{0}}, \varphi_{n_{0}}(x)\right)$ :

$$
\begin{aligned}
& \left\|l_{h} \mathrm{P}_{h} \mathrm{~T}_{h} \prod_{1 h} u(x)-\mathrm{T} u(x)\right\|_{H_{q}\left(\mathbb{R}_{n}\right)} \\
& =\| \mathrm{T}_{h} \prod_{1 h} u(x)-\mathrm{T}_{h} \prod_{1 h} \varphi_{m}(x) \\
& \quad+l_{h} \mathrm{P}_{h} \mathrm{~T}_{h} \prod_{1 h} \varphi_{m}(x)-\mathrm{T} \varphi_{m}(x)+\mathrm{T} \varphi_{m}(x)-\mathrm{T} u(x) \|_{H_{q}\left(\mathbb{R}_{n}\right)} \\
& \leqslant C\left\|\mathrm{~T}_{h} \prod_{1 h}\left(u(x)-\varphi_{m}(x)\right)\right\|_{M(q, h)}+\left\|l_{h} \mathrm{P}_{h} \mathrm{~T}_{h} \prod_{1 h} \varphi_{m}(x)-\mathrm{T} \varphi_{m}(x)\right\|_{H_{q}\left(\mathbb{R}_{n}\right)} \\
& \quad+\left\|\mathrm{T}\left(u(x)-\varphi_{m}(x)\right)\right\|_{H_{q}\left(\mathbb{R}_{n}\right)}<C_{1} \varepsilon_{m}+2 \varepsilon_{m}=k \varepsilon_{m},
\end{aligned}
$$

где константа $k$ не зависит от $h$. Из неравенства (1.23) следует предложение 1.5.

ОПРЕДЕЛЕНИЕ 1.7. Будем говорить, что ограниченное семейство операторов $\mathrm{T}_{h}: M(r, h) \rightarrow M(q, h), r>0, q<0, \prod_{h}^{*}$-аппроксимирует в ограниченной области $\Omega$ ограниченный оператор $\mathrm{T}: H_{r}\left(\mathbb{R}_{n}\right) \rightarrow H_{q}\left(\mathbb{R}_{n}\right)$ конечного порядка, если для любой $\varphi(x) \in C_{0}^{\infty}\left(\mathbb{R}_{n}\right)$ вьполняется равенство

$$
\lim _{|h| \rightarrow 0}\left\|\mathrm{P}_{h} \mathrm{~T}_{h} \prod_{h}^{*} \varphi(x)-\mathrm{P}_{h} \mathrm{~T} \varphi(x)\right\|_{L_{2}\left(\Omega_{1 h}\right)}=0
$$

где оператор $\prod_{h}^{*}$ задается в [2; определение 5.2].

ЗАмечание 1.1. Пусть семейство $\mathrm{T}_{h}: M(r, h) \rightarrow M(q, h), r>0, q<0, \prod_{h}^{*}$-аппроксимирует оператор $\mathrm{T}: H_{r}\left(\mathbb{R}_{n}\right) \rightarrow H_{q}\left(\mathbb{R}_{n}\right)$ в области $\Omega$. Тогда если для некоторого $r_{0}>0$ семейство операторов $\mathrm{T}_{h}$ равномерно ограничено по $h$ из $M\left(r_{0}, h\right)$ в $M(0, h)$, то из [2; предложение 5.9$]$ следует, что семейство $\mathrm{T}_{h}$ аппроксимирует $\mathrm{T}$ в области $\Omega$ (см. определение 1.5 ). 
ПРЕДЛОЖЕНИЕ 1.6. Предположим, что ограниченное семейство операторов $\mathrm{T}_{h}: M(r, h) \rightarrow M(q, h), r>0, q<0, \prod_{h}^{*}$-аппроксимирует в ограниченной области $\Omega$ ограниченный оператор $\mathrm{T}: H_{r}\left(\mathbb{R}_{n}\right) \rightarrow H_{q}\left(\mathbb{R}_{n}\right)$ конечного порядка. Тогда для любой $\varphi(x) \in C_{0}^{\infty}\left(\mathbb{R}_{n}\right)$ существует продолэсение $l_{h}$ функиии $\mathrm{P}_{h} \mathrm{~T}_{h} \prod_{1 h}^{*} \varphi(x)$, которое удовлетворяет условиям:

1. $l_{h} \mathrm{P}_{h} \mathrm{~T}_{h} \prod_{1 h}^{*} \varphi(x)$ является ступенчатой функцией с ячейками постоянства $D(k, h)$ и $a(x, h)=l_{h} \mathrm{P}_{h} \mathrm{~T}_{h} \prod_{1 h}^{*} \varphi(x)=0$ при $x \in D(k, h) \subset \Omega_{2 h} \backslash \bar{\Omega}_{1 h} ;$

2. $\lim _{|h| \rightarrow 0}\left\|l_{h} \mathrm{P}_{h} \mathrm{~T}_{h} \prod_{1 h}^{*} \varphi(x)-\mathrm{T} \varphi(x)\right\|_{L_{2}\left(\mathbb{R}_{n}\right)}=0$,

әде $\prod_{1 h}^{*}$ - сужсние оператора $\prod_{h}^{*}$ на область $\Omega_{1 h}$.

Доказательство аналогично доказательству предложения 1.3.

ПРЕДЛОЖЕНИЕ 1.7. Предположсим, что ограниченное семейство операторов $\mathrm{T}_{h}: M(r, h) \rightarrow M(q, h), r>0, q<0, \prod_{h}^{*}$-аппроксимирует в ограниченной, нормальной, допускающей разбиение области $\Omega$ ограниченный оператор $\mathrm{T}: H_{r}\left(\mathbb{R}_{n}\right) \rightarrow H_{q}\left(\mathbb{R}_{n}\right)$. Тогда для любой $u(x) \in \stackrel{\circ}{H}_{r}(\Omega)$ имеет место соотношение

$$
\lim _{|h| \rightarrow 0}\left\|\mathrm{P}_{1 h} \mathrm{~T}_{h} \prod_{1 h} u(x)-\mathrm{PT} u(x)\right\|_{H_{q}(\Omega)}=0 .
$$

ДоказАтЕльство. Пусть $u(x) \in \stackrel{\circ}{H}_{r}(\Omega)$ - некоторая функция. Возьмем произвольное $\varepsilon>0$, тогда найдется функция $\varphi_{\varepsilon}(x) \in C_{0}^{\infty}(\Omega)$ такая, что

$$
\left\|u(x)-\varphi_{\varepsilon}(x)\right\|_{\stackrel{\circ}{H}_{r}(\Omega)}<\varepsilon .
$$

Из предложения 1.6 следует, что сушествуют функция $l_{h} \mathrm{P}_{h} \mathrm{~T}_{h} \prod_{1 h}^{*} \varphi_{\varepsilon}(x)$ и $\delta\left(\varepsilon, \varphi_{\varepsilon}(x)\right)>0$ такие, что $\forall h,|h|<\delta\left(\varepsilon, \varphi_{\varepsilon}(x)\right)$, будет выполняться неравенство

$$
\left\|\mathrm{T} \varphi_{\varepsilon}(x)-l_{h} \mathrm{P}_{h} \mathrm{~T}_{h} \prod_{1 h}^{*} \varphi_{\varepsilon}(x)\right\|_{L_{2}\left(\mathbb{R}_{n}\right)}<\varepsilon .
$$

Из предложения 5.9 (см. [2]) следует: существует $\delta_{1}\left(\varepsilon, \varphi_{\varepsilon}(x)\right)$ такое, что $\Omega_{1 h} \supset$ $\operatorname{supp} \varphi_{\varepsilon}(x)$ для всех $h,|h|<\delta_{1}\left(\varepsilon, \varphi_{\varepsilon}(x)\right)$, а также

$$
\left\|\prod_{1 h}^{*} \varphi_{\varepsilon}(x)-\prod_{1 h} \varphi_{\varepsilon}(x)\right\|_{M(r, h)}<\varepsilon .
$$

Далее для всех $h,|h|<\delta_{2}=\min \left\{\delta_{1}\left(\varepsilon, \varphi_{\varepsilon}(x)\right), \delta\left(\varepsilon, \varphi_{\varepsilon}(x)\right)\right\}$, получим:

$$
\begin{aligned}
& \left\|\mathrm{P}_{1 h} \mathrm{~T}_{h} \prod_{1 h} u(x)-\mathrm{PT} u(x)\right\|_{H_{q}(\Omega)} \\
& \leqslant\left\|\mathrm{P}_{1 h} \mathrm{~T}_{h} \prod_{1 h} u(x)-\mathrm{P}_{1 h} \mathrm{~T}_{h} \prod_{1 h} \varphi_{\varepsilon}(x)\right\|_{H_{q}(\Omega)} \\
& \quad+\left\|\mathrm{P}_{1 h} \mathrm{~T}_{h} \prod_{1 h} \varphi_{\varepsilon}(x)-\mathrm{P}_{1 h} \mathrm{~T}_{h} \prod_{1 h}^{*} \varphi_{\varepsilon}(x)\right\|_{H_{q}(\Omega)} \\
& \quad+\left\|\mathrm{P}_{1 h} \mathrm{~T}_{h} \prod_{1 h}^{*} \varphi_{\varepsilon}(x)-\mathrm{PT}_{\varepsilon}(x)\right\|_{H_{q}(\Omega)}+\left\|\mathrm{PT} \varphi_{\varepsilon}(x)-\mathrm{PT} u(x)\right\|_{H_{q}(\Omega)} \\
& \quad=\sum_{k=1}^{4} I_{k}(h) .
\end{aligned}
$$


Аналогично, как и при доказательствепредложения 1.4, выводятся неравенства

$$
I_{i}(h)<k \cdot \varepsilon, \quad i=1,3,4,
$$

где $k$ не зависит от $h=\left(h_{1}, \ldots, h_{n}\right)$.

Для оценки $I_{2}(h)$ воспользуемся предложением 6.5 (см. [2]) и (1.27):

$$
\begin{aligned}
I_{2}(h) & =C\left\|\mathrm{P}_{h} \mathrm{~T}_{h}\left(\prod_{1 h} \varphi_{\varepsilon}(x)-\prod_{1 h}^{*} \varphi_{\varepsilon}(x)\right)\right\|_{M\left(q, h, \Omega_{1 h}\right)} \\
& \leqslant C_{1}\left\|\prod_{1 h} \varphi_{\varepsilon}(x)-\prod_{1 h}^{*} \varphi_{\varepsilon}(x)\right\|_{M(r, h)}=C_{1} \varepsilon .
\end{aligned}
$$

Из неравенств (1.29) и (1.30) следует предложение 1.7.

ПРЕДЛОЖЕНИЕ 1.8. Предположим, что ограниченное семейство операторов $\mathrm{T}_{h}: M(r, h) \rightarrow M(q, h), r>0, q<0, \prod_{h}^{*}$-аппроксимирует в ограниченной, нормальной, допускающей разбиение области $\Omega$ ограниченный оператор $\mathrm{T}: H_{r}\left(\mathbb{R}_{n}\right) \rightarrow H_{q}\left(\mathbb{R}_{n}\right)$ конечного порядка. Тогда для любой функиии $u(x) \in \stackrel{\circ}{H}_{r}(\Omega)$ существует продолжсение $l_{h} \mathrm{P}_{h} \mathrm{~T}_{h} \prod_{1 h} u(x) \in M(q, h)$ функиии $\mathrm{P}_{h} \mathrm{~T}_{h} \prod_{1 h} u(x) \in M\left(q, h, \Omega_{1 h}\right)$ maкое, что

$$
\lim _{|h| \rightarrow 0}\left\|l_{h} \mathrm{P}_{h} \mathrm{~T}_{h} \prod_{1 h} u(x)-\mathrm{T} u(x)\right\|_{H_{q}\left(\mathbb{R}_{n}\right)}=0
$$

ДоказАТЕльство. Пусть $u(x) \in \stackrel{\circ}{H}_{r}(\Omega)$ - некоторая функция. Возьмем последовательность $\left\{\varepsilon_{j}>0\right\}_{j=1}^{\infty}, \varepsilon_{j} \searrow 0$. Тогда сушествует такая последовательность $\left\{\varphi_{j}(x)\right\}_{j=1}^{\infty}, \varphi_{j}(x) \in C_{0}^{\infty}(\Omega)$, для которой выполняется условие:

$$
\left\|u(x)-\varphi_{j}(x)\right\|_{\stackrel{\circ}{H}_{r}(\Omega)}<\frac{\varepsilon_{j}}{\|\mathrm{~T}\|} .
$$

Изпредложения 1.6 следует: найдутся последовательность $\left\{\delta_{j}\left(\varepsilon_{j}, \varphi_{j}(x)\right)>0\right\}_{j=1}^{\infty}$, $\delta_{j}\left(\varepsilon_{j}, \varphi_{j}(x)\right) \searrow 0$, и продолжения $l_{h} \mathrm{P}_{h} \mathrm{~T}_{h} \prod_{1 h}^{*} \varphi_{j}(x)$ такие, что $\Omega_{1 h} \supset \operatorname{supp} \varphi_{j}(x)$ для всех $h,|h|<\delta_{j}\left(\varepsilon_{j}, \varphi_{j}(x)\right)$, и для каждой фиксированной функции $\varphi_{j}(x), \mathrm{a}$ также

$$
\left\|l_{h} \mathrm{P}_{h} \mathrm{~T}_{h} \prod_{1 h}^{*} \varphi_{j}(x)-\mathrm{T} \varphi_{j}(x)\right\|_{H_{q}\left(\mathbb{R}_{n}\right)}<\varepsilon_{j}
$$

Возьмем какое-нибудь $h$, удовлетворяющее неравенству $\delta_{j+1}\left(\varepsilon_{j+1}, \varphi_{j+1}(x)\right) \leqslant$ $|h|<\delta_{j}\left(\varepsilon_{j}, \varphi_{j}(x)\right)$, тогда продолжение $l_{h} \mathrm{P}_{h} \mathrm{~T}_{h} \prod_{1 h} u(x)$ определим соотношениem:

$$
l_{h} \mathrm{P}_{h} \mathrm{~T}_{h} \prod_{1 h} u(x)=\mathrm{T}_{h} \prod_{1 h} u(x)-\mathrm{T}_{h} \prod_{1 h} \varphi_{j}(x)+l_{h} \mathrm{P}_{h} \mathrm{~T}_{h} \prod_{1 h}^{*} \varphi_{j}(x)
$$


Из конструкции $l_{h} \mathrm{P}_{h} \mathrm{~T}_{h} \prod_{1 h}^{*}$, которая совпадает с указанной в предложении 1.3 , следует, что $\mathrm{P}_{h} l_{h} \mathrm{P}_{h} \mathrm{~T}_{h} \prod_{1 h} u(x)=\mathrm{P}_{h} \mathrm{~T}_{h} \prod_{1 h} u(x)$ и, следовательно, продолжение, задаваемое (1.34), корректно. Пусть $|h|<\delta_{n_{0}}\left(\varepsilon_{n_{0}}, \varphi_{n_{0}}(x)\right)$, тогда, используя теорему 2.1 (см. [2]), при $\delta_{m+1}\left(\varepsilon_{m+1}, \varphi_{m+1}(x)\right) \leqslant|h|<\delta_{m}\left(\varepsilon_{m}, \varphi_{m}(x)\right)<$ $\delta_{n_{0}}\left(\varepsilon_{n_{0}}, \varphi_{n_{0}}(x)\right)$ получаем:

$$
\begin{aligned}
& \left\|l_{h} \mathrm{P}_{h} \mathrm{~T}_{h} \prod_{1 h} u(x)-\mathrm{T} u(x)\right\|_{H_{q}\left(\mathbb{R}_{n}\right)} \\
& =\| \mathrm{T}_{h} \prod_{1 h} u(x)-\mathrm{T}_{h} \prod_{1 h} \varphi_{m}(x)+l_{h} \mathrm{P}_{h} \mathrm{~T}_{h} \prod_{1 h}^{*} \varphi_{m}(x) \\
& \quad-\mathrm{T} \varphi_{m}(x)+\mathrm{T} \varphi_{m}(x)-\mathrm{T} u(x) \|_{H_{q}\left(\mathbb{R}_{n}\right)} \\
& \leqslant \\
& \quad C \cdot\left\|\mathrm{T}_{h} \prod_{1 h}\left(u(x)-\varphi_{m}(x)\right)\right\|_{M(q, h)} \\
& \quad+\left\|l_{h} \mathrm{P}_{h} \mathrm{~T}_{h} \prod_{1 h}^{*} \varphi_{m}(x)-\mathrm{T} \varphi_{m}(x)\right\|_{H_{q}\left(\mathbb{R}_{n}\right)}+\|\mathrm{T}\| \cdot\left\|\varphi_{m}(x)-u(x)\right\|_{\stackrel{\circ}{r}_{r}\left(\mathbb{R}_{n}\right)} \\
& <k \varepsilon_{n_{0}},
\end{aligned}
$$

где $k$ не зависит от $h$. Из (1.35) следует предложение 1.8 .

ОПРЕДЕЛЕНИЕ 1.8. Будем говорить, что ограниченное семейство операторов $\mathrm{T}_{h}$, отображающих $\stackrel{\circ}{M}\left(r, h, \Omega_{1 h}\right)$ в $M(q, h), r>0, q<0, \prod_{h}^{*}$-аппроксимирует в ограниченной области $\Omega$ ограниченный оператор $\mathrm{T}: \stackrel{\circ}{H}_{r}(\Omega) \rightarrow H_{q}\left(\mathbb{R}_{n}\right)$ конечного порядка, если для любой $\varphi(x) \in C_{0}^{\infty}(\Omega)$ вьполняется соотношение:

$$
\lim _{|h| \rightarrow 0}\left\|\mathrm{P}_{h} \mathrm{~T}_{h} \prod_{1 h} u(x)-\mathrm{P}_{h} \mathrm{~T} \varphi(x)\right\|_{L_{2}\left(\Omega_{1 h}\right)}=0 .
$$

ЗАМЕЧАНИЕ 1.2. Если в нормальной, допускающей разбиение области $\Omega$ ограниченное семейство операторов $\mathrm{T}_{h}$ из $\stackrel{\circ}{M}\left(r, h, \Omega_{1 h}\right)$ в $M(q, h), r>0, q<0$, $\prod_{h}^{*}$-аппроксимирует ограниченный оператор Т конечного порядка, отображающий $\stackrel{\circ}{H}_{r}(\Omega)$ в $H_{q}\left(\mathbb{R}_{n}\right)$, то, как видно из доказательств предложений $1.3-1.8$, эти предложения остаются в силе для вышеуказанного семейства $\mathrm{T}_{h}$ и оператора $\mathrm{T}$.

\section{§2. Сходимость приближенных решений и существование решений операторных уравнений}

ОПрЕДЕЛЕНиЕ 2.1. Ограниченное семейство операторов $\mathrm{T}_{h}$ из $\stackrel{\circ}{M}\left(r, h, \Omega_{1 h}\right)$ в $M(-r, h), r>0$, называется устойчивым, если сушествуют числа $\delta_{0}>0$ и $C>0$ такие, что для любой $a(x, h) \in \stackrel{\circ}{M}\left(r, h, \Omega_{1 h}\right)$ и для всех $h,|h|<\delta_{0}$, выполняется оценка

$$
\left\|\mathrm{P}_{h} \mathrm{~T}_{h} a(x, h)\right\|_{M\left(-r, h, \Omega_{1 h}\right)} \geqslant C\|a(x, h)\|_{\stackrel{\circ}{\circ\left(r, h, \Omega_{1 h}\right)}},
$$

где $\mathrm{P}_{h}$ - оператор сужения на $\Omega_{1 h}, C$ не зависит от $h=\left(h_{1}, \ldots, h_{n}\right)$. 
ОПРЕДЕЛЕНиЕ 2.2. Семейство функций $a(x, h) \in \stackrel{\circ}{M}\left(r, h, \Omega_{1 h}\right), r \geqslant 0$, называется сходящимся в $\stackrel{\circ}{M}\left(r, h, \Omega_{1 h}\right)$ к функции $u(x) \in \stackrel{\circ}{H}_{r}(\Omega)$, если имеет место равенство

$$
\lim _{|h| \rightarrow 0}\left\|\prod_{1 h} u(x)-a(x, h)\right\|_{\stackrel{\circ}{\left(r, h, \Omega_{1 h}\right)}}=0 .
$$

ОПРЕДЕЛЕНИЕ 2.3. Семейство функций $a(x, h) \in \stackrel{\circ}{M}\left(r, h, \Omega_{1 h}\right), r \geqslant 0$, называется слабо сходящимся в $\stackrel{\circ}{M}\left(r, h, \Omega_{1 h}\right)$ к функции $u(x) \in \stackrel{\circ}{H}_{r}(\Omega)$, если имеет место соотношение

$$
\lim _{|h| \rightarrow 0}\left\|\prod_{1 h} u(x)-a(x, h)\right\|_{\stackrel{\circ}{M\left(r_{0}, h, \Omega_{1 h}\right)}}=0
$$

для любого $r_{0}<r$.

ПРЕДЛОЖЕНИЕ 2.1. Пусть устойчивое семейство операторов $\mathrm{T}_{h}$, отображсающее $M(r, h)$ на $M(-r, h)$, слабо аппроксимирует ограниченный оператор $\mathrm{T}$ из $H_{r}\left(\mathbb{R}_{n}\right)$ в $H_{-r}\left(\mathbb{R}_{n}\right), r>0$, и пусть, кроме того, для любой функиии $f(x) \in H_{-r}\left(\mathbb{R}_{n}\right)$ уравнение $\mathrm{T} u=f$ имеет решение. Тогда справедливь следующие утверждения:

1. решение уравнения Т $u=f$ единственно;

2. функции $a(x, h) \in M(r, h)$, являющиеся решениями уравнений $\mathrm{T}_{h} a(x, h)$ $=f(x, h), f(x, h) \in M(-r, h), \lim _{|h| \rightarrow 0}\|f(x)-f(x, h)\|_{H_{-r}\left(\mathbb{R}_{n}\right)}=0$, cxoдятся к решению уравнения Т $u=f$ в $M(r, h)$.

ДокАЗАТЕЛЬство. Пусть функции $u_{1}(x), u_{2}(x) \in H_{r}\left(\mathbb{R}_{n}\right)$ являются решениями уравнения $\mathrm{T} u=f$. Тогда из предложения 1.1 следует, что $\mathrm{T}_{h} \prod_{h} u_{1}(x) \rightarrow f(x)$, $\mathrm{T}_{h} \prod_{h} u_{2}(x) \rightarrow f(x)$ по норме $H_{-r}\left(\mathbb{R}_{n}\right)$ при $|h| \rightarrow 0$. Отсюда по теореме 2.1 $\mathrm{T}_{h} \prod_{h}\left(u_{1}(x)-u_{2}(x)\right) \rightarrow 0$ по норме $M(-r, h)$ при $|h| \rightarrow 0$. Из устойчивости семейства $\mathrm{T}_{h}$ вытекает, что $\prod_{h}\left(u_{1}(x)-u_{2}(x)\right) \rightarrow 0$ по норме $M(r, h)$ при $|h| \rightarrow 0$. Используя предложение 5.11 (см. [2]), получаем $u_{1}(x)=u_{2}(x)$. Единственность решения $\mathrm{T} u=f$ доказана.

Так как операторы $\mathrm{T}_{h}$ отображают $M(r, h)$ на $M(-r, h)$, то решение уравнения $\mathrm{T}_{h} a(x, h)=f(x, h)$ существует. Семейство $\mathrm{T}_{h}$ устойчиво, поэтому существует $\delta>0$ такое, что при $|h|<\delta$ уравнение имеет единственное решение. Из теоремы 2.1 (см. [2]) и предложения 1.1 имеем: $\mathrm{T}_{h}\left(a(x, h)-\prod_{h} u(x)\right) \rightarrow 0$ по норме $M(-r, h)$ при $|h| \rightarrow 0$. Отсюда и из устойчивости семейства $\mathrm{T}_{h}$ следует, что

$$
\lim _{|h| \rightarrow 0}\left\|\prod_{h} u(x)-a(x, h)\right\|_{M(r, h)}=0 .
$$

Предложение 2.1 доказано.

ПРЕДЛОЖЕНИЕ 2.2. Пусть в нормальной, допускающей разбиение области $\Omega$ устойчивое семейство операторов $\mathrm{T}_{h}: \stackrel{\circ}{M}\left(r, h, \Omega_{1 h}\right) \rightarrow M(-r, h), r>0$, $\prod_{h}^{*}$-аппроксимирует ограниченный оператор $\mathrm{T}$ конечного порядка, отображающий $\stackrel{\circ}{H}_{r}(\Omega)$ в $H_{-r}(\Omega)$. Если для любой функиии $f(x) \in H_{-r}(\Omega)$ уравнение $\mathrm{PT} u=f$ имеет решение $(\mathrm{P}$ - оператор сужения на область $\Omega)$, то:

1. решение уравнения $\mathrm{PT} u=f$ единственно; 
2. решения $a(x, h) \in \stackrel{\circ}{M}\left(r, h, \Omega_{1 h}\right)$ уравнений $\mathrm{P}_{h} \mathrm{~T}_{h} a(x, h)=\mathrm{P}_{h} f(x, h)$, $f(x, h) \in M(-r, h), \lim _{|h| \rightarrow 0}\|l f(x)-f(x, h)\|_{H_{-r}\left(\mathbb{R}_{n}\right)}=0$, сходятся $\kappa$ решению $и(x)$ уравнения $\mathrm{PT} u=f(x)$ в $\stackrel{\circ}{M}\left(r, h, \Omega_{1 h}\right)$, әде $\mathrm{P}_{h}$ - оператор сужения на $\Omega_{1 h}, l f(x)$ - некоторое продолжение $f(x) \in H_{-r}(\Omega)$ на $\mathbb{R}_{n}, l f(x) \in H_{-r}\left(\mathbb{R}_{n}\right)$.

ДокАЗАТЕЛЬСТВо. Пусть функции $u_{1}(x) \in \stackrel{\circ}{H}_{r}(\Omega)$ и $u_{2}(x) \in \stackrel{\circ}{H}_{r}(\Omega)$ являются решениями уравнения $\mathrm{PT} u=f, f(x) \in H_{-r}(\Omega)$. Тогда по замечанию 1.2 существуют такие продолжения $l_{1 h} \mathrm{P}_{h} \mathrm{~T}_{h} \prod_{1 h} u_{1}(x)$ и $l_{2 h} \mathrm{P}_{h} \mathrm{~T}_{h} \prod_{1 h} u_{2}(x)$ функций $l_{h} \mathrm{P}_{h} \mathrm{~T}_{h} \prod_{1 h} u_{1}(x)$ и $l_{h} \mathrm{P}_{h} \mathrm{~T}_{h} \prod_{1 h} u_{2}(x)$, что $l_{1 h} \mathrm{P}_{h} \mathrm{~T}_{h} \prod_{1 h} u_{1}(x) \rightarrow \mathrm{T} u_{1}(x)$ и $l_{2 h} \mathrm{P}_{h} \mathrm{~T}_{h} \prod_{1 h} u_{2}(x) \rightarrow \mathrm{T} u_{2}(x)$ по норме $H_{-r}\left(\mathbb{R}_{n}\right)$ при $|h| \rightarrow 0$. Из предложения 6.12 (см. [2]) следует: $\mathrm{P}_{h} \mathrm{~T}_{h} \prod_{1 h}\left(u_{1}(x)-u_{2}(x)\right) \rightarrow 0$ по норме $M\left(-r, h, \Omega_{1 h}\right)$ при $|h| \rightarrow 0$. Отсюда и из устойчивости семейства $\mathrm{T}_{h}$ имеем: $\prod_{1 h}\left(u_{1}(x)-u_{2}(x)\right) \rightarrow 0$ по норме $\stackrel{\circ}{M}\left(r, h, \Omega_{1 h}\right)$ при $|h| \rightarrow 0$. Используя предложение 6.9 (см. [2]), получаем $u_{1}(x)=u_{2}(x)$. Единственность решения уравнения $\mathrm{PT} u=f(x)$ доказана.

Пространства $\stackrel{\circ}{M}\left(r, h, \Omega_{1 h}\right)$ и $M\left(-r, h, \Omega_{1 h}\right)$ конечномерны и имеют одинаковую размерность. Семейство операторов $\mathrm{T}_{h}$ устойчиво, поэтому найдется такое $\delta>0$, что при $|h|<\delta$ сушествует единственное решение уравнения $\mathrm{P}_{h} \mathrm{~T}_{h} a(x, h)=$ $\mathrm{P}_{h} f(x, h)$.

По замечанию 1.2 сушествует такое продолжение $l_{h} \mathrm{P}_{h} \mathrm{~T}_{h} \prod_{1 h} u(x)$ функции $\mathrm{P}_{h} \mathrm{~T}_{h} \prod_{1 h} u(x)$, что $l_{h} \mathrm{P}_{h} \mathrm{~T}_{h} \prod_{1 h} u(x) \rightarrow \mathrm{T} u(x)$ по норме $H_{-r}\left(\mathbb{R}_{n}\right)$ при $|h| \rightarrow 0$.

Из предложения 6.12 (см. [2]) следует, что $\mathrm{P}_{h} \mathrm{~T}_{h}\left(\prod_{1 h} u(x)-a(x, h)\right) \rightarrow 0$ по норме $M\left(-r, h, \Omega_{1 h}\right)$ при $|h| \rightarrow 0$. Отсюда и из устойчивости семейства $\mathrm{T}_{h}$ вытекает $\left\|\prod_{1 h} u(x)-a(x, h)\right\|_{\stackrel{\circ}{M}\left(r, h, \Omega_{1 h}\right)} \rightarrow 0$ при $|h| \rightarrow 0$. Предложение 2.2 доказано.

ПРЕДЛОЖЕНИЕ 2.3. Пусть в нормальной, допускающей разбиение области $\Omega$ устойчивое семейство операторов $\mathrm{T}_{h}: \stackrel{\circ}{M}\left(r, h, \Omega_{1 h}\right) \rightarrow M(-r, h), r>0$, $\prod_{h}^{*}$-аппроксимирует ограниченный оператор $\mathrm{T}: \stackrel{\circ}{H}{ }_{r}(\Omega) \rightarrow H_{r}(\Omega)$ конечного порядка и при некотором $r_{0}<r$ семейство операторов $\mathrm{T}_{h}$ ограничено из $\stackrel{\circ}{M}\left(r_{0}, h, \Omega_{1 h}\right)$ в $M(q, h)$. Тогда существует и единственно решение уравнения $\mathrm{PT} u=f$ для любой $f \in H_{-r}(\Omega)\left(\right.$ здесь $u(x) \in \stackrel{\circ}{H}_{r}(\Omega), \mathrm{P}-$ оператор сужения на $\Omega$ ).

ДокАЗАТЕльство. Единственность решения следует из предложения 2.2. Равенство РТ $u=f$ понимается как равенство двух линейных функционалов над пространством $\stackrel{\circ}{H}_{r}(\Omega)$, т.е. для любой функции $\nu(x) \in \stackrel{\circ}{H}_{r}(\Omega)$ должно вьполняться

$$
(\nu, \mathrm{PT} u)=(\nu, f)
$$

где $(\nu, \mathrm{PT} u)$ и $(\nu, f)$ - обозначения линейных функционалов, определяемых функциями РТ $u(x)$ и $f(x)$. Нужно отметить, что эти функционалы не зависят от продолжения РТ и $f\left(\right.$ см. [3]) как линейных функционалов на $H_{r}\left(\mathbb{R}_{n}\right)$. В качестве продолжения $\mathrm{PT} u$ возьмем $\mathrm{T} u$, а в качестве продолжения $f$ возьмем то, которое обладает минимальной нормой в $H_{-r}\left(\mathbb{R}_{n}\right)$, оно сушествует, поскольку $H_{-r}\left(\mathbb{R}_{n}\right)$ является 
гильбертовым пространством, обозначать это продолжение будем через $l f$. Итак, под решением $u(x)$ уравнения РТ $u=f$ понимается функция $u(x) \in \stackrel{\circ}{H}{ }_{r}(\Omega)$ такая, что для любой функции $\nu(x) \in \stackrel{\circ}{H}_{r}(\Omega)$ выполняется равенство

$$
(\nu, \mathrm{T} u)=(\nu, l f)
$$

Согласно предложению 6.8 (см. [2]) сушествует семейство функций $f(x, h) \in$ $M(-r, h)$ такое, что $f(x, h) \rightarrow l f(x)$ по норме $H_{-r}\left(\mathbb{R}_{n}\right), \mathrm{P}_{1 h} f(x, h) \rightarrow f(x)$ по норме $H_{-r}(\Omega)$ при $|h| \rightarrow 0$, где $\mathrm{P}_{1 h}$ - оператор сужения на $\Omega$ (см. определение 1.6$)$. Рассмотрим семейство уравнений

$$
\mathrm{P}_{h} \mathrm{~T}_{h} a(x, h)=\mathrm{P}_{h} f(x, h),
$$

где $\mathrm{P}_{h}$ - оператор сужения на область $\Omega_{1 h}$.

Так как семейство операторов $\mathrm{T}_{h}$ устойчиво, то существует такое число $\delta>0$, что при всех $h,|h|<\delta$, решения $a(x, h)$ уравнений $(2.3)$ сушествуют и единственны. Кроме того, выполняется неравенство $\|a(x, h)\|_{M\left(r, h, \Omega_{1 h}\right)}^{\circ}<C\|f(x)\|_{H_{-r}(\Omega)}$. Отсюда согласно предложению 6.2 из [2] существуют последовательность $\left\{h^{n}\right\}_{n=1}^{\infty}$, $\left|h^{n}\right| \rightarrow 0$ при $n \rightarrow \infty$, число $r_{0}, 0<r_{0}<\frac{1}{2}, r_{0}<r$, и функция $a(x) \in \stackrel{\circ}{H}{ }_{r}(\Omega)$ такие, что $a\left(x, h^{n}\right) \rightarrow a(x)$ по норме $\stackrel{\circ}{H}_{r_{0}}(\Omega)$ при $\left|h^{n}\right| \rightarrow 0$. Докажем, что функция $a(x)$ удовлетворяет соотношению (2.2). Согласно замечанию 1.2 (см. предложение 1.7) для РТ $a(x)$, сужения линейного функционала Т $a(x)$ на $\stackrel{\circ}{H} \underset{r}{ }(\Omega)$, имеет место представление

$$
\operatorname{PT} a(x)=\mathrm{P}_{1 h^{n}} \mathrm{~T}_{h^{n}} \prod_{1 h^{n}} a(x)+\varepsilon\left(x, h^{n}\right),
$$

где $\varepsilon\left(x, h^{n}\right) \rightarrow 0$ по норме $H_{-r}(\Omega)$ при $\left|h^{n}\right| \rightarrow 0$. Отсюда вытекает

$$
(\nu, \mathrm{T} a(x))=\lim _{\left|h^{n}\right| \rightarrow 0}\left(\nu(x), \mathrm{P}_{1 h^{n}} \mathrm{~T}_{h^{n}} \prod_{1 h^{n}} a(x)\right) .
$$

Далее имеем:

$$
\begin{aligned}
(\nu, \mathrm{T} a(x))= & \lim _{\left|h^{n}\right| \rightarrow 0}\left(\nu, \mathrm{P}_{1 h^{n}} \mathrm{~T}_{h^{n}} a\left(x, h^{n}\right)\right) \\
& +\lim _{\left|h^{n}\right| \rightarrow 0}\left(\nu(x), \mathrm{P}_{1 h^{n}} \mathrm{~T}_{h^{n}}\left(\prod_{1 h^{n}} a(x)-a\left(x, h^{n}\right)\right)\right) \\
= & \lim _{\left|h^{n}\right| \rightarrow 0}\left(\nu, \mathrm{P}_{1 h^{n}} f\left(x, h^{n}\right)\right) \\
& +\lim _{\left|h^{n}\right| \rightarrow 0}\left(\prod_{1 h^{n}} \nu(x), \mathrm{P}_{h^{n}} \mathrm{~T}_{h^{n}}\left(\prod_{1 h^{n}} a(x)-a\left(x, h^{n}\right)\right)\right) \\
= & (\nu, l f(x))+\lim _{\left|h^{n}\right| \rightarrow 0} I\left(h^{n}\right) .
\end{aligned}
$$


Из предложения 6.14 (см. [2]) следует $\left\|\prod_{1 h^{n}} a(x)-a\left(x, h^{n}\right)\right\|_{M\left(r_{1}, h^{n}, \Omega_{\left.1 h^{n}\right)}\right.} \rightarrow 0$ для любого $r_{1}<r$. По условию предложения 2.3 сушествует такое $r_{0}<r$, что семейство операторов $\mathrm{T}_{h^{n}}$ будет ограниченным из $\stackrel{\circ}{M}\left(r_{0}, h^{n}, \Omega_{1 h^{n}}\right)$ в $M\left(q, h^{n}\right)$. Отсюда при $\left|h^{n}\right| \rightarrow 0$ имеем:

$$
\begin{aligned}
& \left\|\mathrm{P}_{h^{n}} \mathrm{~T}_{h^{n}}\left(\prod_{1 h^{n}} a(x)-a\left(x, h^{n}\right)\right)\right\|_{M\left(q, h^{n}, \Omega_{1 h^{n}}\right)} \\
& \quad \leqslant\left\|\mathrm{T}_{h^{n}}\left(\prod_{1 h^{n}} a(x)-a\left(x, h^{n}\right)\right)\right\|_{M\left(q, h^{n}\right)} \\
& \quad \leqslant\left\|\mathrm{T}_{h^{n}}\right\|_{r_{0}, q} \cdot\left\|\prod_{1 h^{n}} a(x)-a\left(x, h^{n}\right)\right\|_{M\left(r_{0}, h^{n}, \Omega_{\left.1 h^{n}\right)}\right.} \rightarrow 0,
\end{aligned}
$$

где $\left\|\mathrm{T}_{h^{n}}\right\|_{r_{0}, q}-$ норма оператора $\mathrm{T}_{h^{n}}: \stackrel{\circ}{M}\left(r_{0}, h^{n}, \Omega_{1 h^{n}}\right) \rightarrow M\left(q, h^{n}\right)$. При $q \geqslant-r$ для $I\left(h^{n}\right)$ (см. (2.6)) выполняются неравенства:

$$
\begin{aligned}
& \left|I\left(h^{n}\right)\right| \leqslant\left\|\prod_{1 h^{n}} \nu(x)\right\|_{\stackrel{\circ}{M\left(r, h^{n}, \Omega_{1 h^{n}}\right)}} \\
& \times\left\|\mathrm{P}_{h^{n}} \mathrm{~T}_{h^{n}}\left(\prod_{1 h^{n}} a(x)-a\left(x, h^{n}\right)\right)\right\|_{M\left(-r, h^{n}, \Omega_{1 h^{n}}\right)} \\
& \leqslant C\|\nu(x)\|_{H_{H^{\prime}}(\Omega)} \cdot\left\|\mathrm{P}_{h^{n}} \mathrm{~T}_{h^{n}}\left(\prod_{1 h^{n}} a(x)-a\left(x, h^{n}\right)\right)\right\|_{M\left(q, h^{n}, \Omega_{1 h^{n}}\right)} .
\end{aligned}
$$

Из (2.7) и (2.8) следует, что $I\left(h^{n}\right) \rightarrow 0$ при $\left|h^{n}\right| \rightarrow 0$.

Если $q<-r$, то из предложения 6.13 (см. [2]) вытекает $\lim _{\left|h^{n}\right| \rightarrow 0} I\left(h^{n}\right)=0$.

Из последних замечаний и соотношения (2.6) получаем, что функция $a(x)$ является решением уравнения РТ $u=f(x)$. Предложение 2.3 доказано.

ОПРЕДЕЛЕНИЕ 2.4. Ограниченное семейство операторов $\mathrm{T}_{h}: \stackrel{\circ}{M}\left(r, h, \Omega_{1 h}\right) \rightarrow$ $M(-r, h), r>0$, будем назьвать слабо сходящимся, если для любой функции $\nu(x) \in \stackrel{\circ}{H}_{r}(\Omega)$ сушествуют продолжения $l_{h}\left(\mathrm{P}_{h} \mathrm{~T}_{h}\right)^{*} \prod_{1 h} \nu(x) \in M(-r, h)$ функций $\left(\mathrm{P}_{h} \mathrm{~T}_{h}\right)^{*} \prod_{1 h} \nu(x) \in M\left(-r, h, \Omega_{1 h}\right)$ и функция $u(x) \in H_{-r}\left(\mathbb{R}_{n}\right)$ такие, что $l_{h}\left(\mathrm{P}_{h} \mathrm{~T}_{h}\right)^{*} \prod_{1 h} \nu(x) \rightarrow u(x)$ по норме $H_{-r}\left(\mathbb{R}_{h}\right)$ при $|h| \rightarrow 0$, где $\left(\mathrm{P}_{h} \mathrm{~T}_{h}\right)^{*}$ - семейство сопряженных к $\mathrm{P}_{h} \mathrm{~T}_{h}$ операторов.

ПРЕДЛОЖЕНИЕ 2.4. Пусть в нормальной, допускающей разбиение области $\Omega$ слабо сходящееся устойчивое семейство операторов $\mathrm{T}_{h}: \stackrel{\circ}{M}\left(r, h, \Omega_{1 h}\right) \rightarrow$ $M(-r, h), r>0, \prod_{h}^{*}$-аппроксимирует ограниченный оператор $\mathrm{T}: \stackrel{\circ}{H}_{r}(\Omega) \rightarrow$ $H_{-r}(\Omega)$ конечного порядка. Тогда существует единственное решение $и(x) \in$ $\stackrel{\circ}{H}_{r}(\Omega)$ уравнения $\mathrm{PT} u=f$ для любой $f \in H_{-r}(\Omega)$.

ДокАЗАТЕЛЬСтво. Рассуждая как и в предложении 2.3 , получаем соотношение (2.6):

$$
(\nu, \mathrm{T} a(x))=(\nu, l f)+\lim _{|h| \rightarrow 0} I(h),
$$

где для простоты вместо $a\left(x, h^{n}\right)$ оставлено обозначение $a(x, h)$, а вместо $h^{n}-$ обозначение $h$. 
Так как по условию предложения 2.4 существует продолжение

$$
l_{h}\left(\mathrm{P}_{h} \mathrm{~T}_{h}\right)^{*} \prod_{1 h} \nu(x) \in M(-r, h)
$$

функции

$$
\left(\mathrm{P}_{h} \mathrm{~T}_{h}\right)^{*} \prod_{1 h} \nu(x) \in M\left(-r, h, \Omega_{1 h}\right)
$$

а функция $\left(\prod_{1 h} a(x)-a(x, h)\right) \in \stackrel{\circ}{M}\left(-r, h, \Omega_{1 h}\right)$, то имеет место соотношение

$$
I(h)=\overline{\left(\prod_{1 h} a(x)-a(x, h), l_{h}\left(\mathrm{P}_{h} \mathrm{~T}_{h}\right)^{*} \prod_{1 h} \nu(x)\right)},
$$

где черта - символ комплексного сопряжения.

Из предложения 7.4 (см. [2]) следует: существует функция $w^{+}(x)$ такая, что выполняется неравенство

$$
\left\|l_{h}\left(\mathrm{P}_{h} \mathrm{~T}_{h}\right)^{*} \prod_{1 h} \nu(x)\right\|_{M\left(-r, h, w^{+}\right)} \leqslant C
$$

где $C$ не зависит от $h$.

Используя предложение 7.1 (см. [2]), получаем:

$$
\lim _{|h| \rightarrow 0}\left\|\prod_{1 h} a(x)-a(x, h)\right\|_{M\left(r, h, 1 / w^{+}\right)}=0
$$

Из предложения 7.5 (см. [2]), а также из (2.10)-(2.12) имеем $\lim _{|h| \rightarrow 0} I(h)=0$. Отсюда и из (2.8) следует предложение 2.4 .

СлЕДСТВИЕ 2.1. Пусть для семейства операторов $\mathrm{T}_{h}$ и оператора $\mathrm{T}$ выполняются условия предложсения 2.3 или 2.4. Рассмотрим семейство уравнений

$$
\mathrm{P}_{h} \mathrm{~T}_{h} a(x, h)=f(x, h),
$$

где $f(x, h) \in M\left(-r, h, \Omega_{1 h}\right),\|f(x, h)\|_{M\left(-r, h, \Omega_{1 h}\right)} \leqslant C, C$ не зависит от $h$. Причем функции $\widetilde{f}(x, h) \rightarrow f(x) \in H_{-r}(\Omega)$ по норме $H_{-r}(\Omega)$ nри $|h| \rightarrow 0$, где $\tilde{f}(x, h)$ определяются следующим образом: $\tilde{f}(x, h)=f(x, h)$ при $x \in D(k, h) \subset \Omega_{1 h}, \tilde{f}(x, h)=0$ nри $x \in \Omega \backslash \bar{\Omega}_{1 h} . \quad$ Тогда существует $\delta>0$ такое, что при $|h|<\delta$ решения уравнений (2.13) существуют и единственнь и функции $a(x, h) \in \stackrel{\circ}{M}\left(r, h, \Omega_{1 h}\right)$ слабо сходятся $к$ решению $а(x)$ уравнения $\mathrm{PT} a(x)=f(x)$, т.е. для любого $r_{0}<r$ выполняется соотношение

$$
\lim _{|h| \rightarrow 0}\left\|\prod_{1 h} a(x)-a(x, h)\right\|_{\stackrel{\circ}{M\left(r_{0}, h, \Omega_{1 h}\right)}}=0
$$

Доказательство следствия 2.1 непосредственно следует из доказательств предложений 2.3 и 2.4 , а также из предложения 2.1 . 
ПРЕДЛОЖЕНИЕ 2.5. Пусть для семейства операторов $\mathrm{T}_{h}$ и оператора $\mathrm{T}$ выполняются условия предложсения 2.3 или 2.4. Кроме того, пусть в нормальной, допускающей разбиение области $\Omega$ ограниченное семейство операторов $\mathrm{F}_{h}$ из $\stackrel{\circ}{M}\left(s, h, \Omega_{1 h}\right), 0<r_{0}<s \leqslant r$, в $M(-r+\varepsilon, h), \varepsilon>0, \prod_{h}^{*}$-anпроксимирует ограниченный оператор $\mathrm{F}$ конечного порядка, отображсющий $\stackrel{\circ}{H}_{s}(\Omega)$ в $H_{-r+\varepsilon}\left(\mathbb{R}_{n}\right)$, а сужение $\mathrm{PA}$ оператора $\mathrm{A}=\mathrm{T}+\mathrm{F}$ на $\stackrel{\circ}{H}_{r}(\Omega)$ имеет ядро, состоящее из одного нулевого әлемента. Тогда существует $\delta>0$ такое, что при $|h|<\delta$ семейство операторов $\mathrm{P}_{h} \mathrm{~A}_{h}=\mathrm{P}_{h} \mathrm{~T}_{h}+\mathrm{P}_{h} \mathrm{~F}_{h}$ устойчиво отображсает $\stackrel{\circ}{M}\left(r, h, \Omega_{1 h}\right)$ в $M\left(-r, h, \Omega_{1 h}\right)$.

ДокАЗАТЕльство. Предположим противное. Тогда найдется последовательность $a\left(x, h^{n}\right) \in \stackrel{\circ}{M}\left(r, h^{n}, \Omega_{1 h^{n}}\right),\left\|a\left(x, h^{n}\right)\right\|_{M\left(r, h^{n}, \Omega_{\left.1 h^{n}\right)}\right.}=1,\left|h^{n}\right| \rightarrow 0$ при $n \rightarrow \infty$,
такая, что

$$
\lim _{\left|h^{n}\right| \rightarrow 0}\left\|\mathrm{P}_{h^{n}} \mathrm{~A}_{h^{n}} a\left(x, h^{n}\right)\right\|_{M\left(-r, h^{n}, \Omega_{1 h^{n}}\right)}=0 .
$$

Обозначим $b\left(x, h^{n}\right)=\mathrm{P}_{1 h^{n}} \mathrm{~F}_{h^{n}} a\left(x, h^{n}\right)$, где $\mathrm{P}_{1 h^{n}}$ - оператор сужения на область $\Omega$ (см. определение 1.6). Из свойств операторов $\mathrm{F}_{h}$ и предложения 6.5 (см. [2]) следуют неравенства $\left\|b\left(x, h^{n}\right)\right\|_{M\left(-r+\varepsilon, h^{n}, \Omega_{1 h^{n}}\right)} \leqslant C,\left\|b\left(x, h^{n}\right)\right\|_{H_{-r+\varepsilon}(\Omega)} \leqslant C_{1}$, где константы $C$ и $C_{1}$ не зависят от $h$. Так как $H_{-r+\varepsilon}(\Omega)$ компактно вложено в $H_{m}(\Omega)$ (см. [3]), $-r<m<-r+\varepsilon$, то из последовательности $b\left(x, h^{n}\right)$ можно выделить подпоследовательность, сходящуюся в $H_{m}(\Omega)$ к функции $b(x) \in H_{m}(\Omega)$. Для этой подпоследовательности оставим обозначение $b\left(x, h^{n}\right)$. Докажем, что $b(x) \neq 0$. Действительно, если $b(x)$ - нулевой элемент $H_{m}(\Omega)$, то из предложения 6.15 (см. [2]) следует, что $\mathrm{P}_{1 h^{n}} \mathrm{~F}_{h^{n}} a\left(x, h^{n}\right) \rightarrow 0$ по норме $M\left(-r, h^{n}, \Omega_{1 h^{n}}\right)$ при $\left|h^{n}\right| \rightarrow 0$.

Последний факт противоречит устойчивости семейства операторов $\mathrm{T}_{h}$ и равенству (2.14). Итак, $b(x) \neq 0$.

Из (2.14) следует равенство

$$
\mathrm{P}_{h^{n}} \mathrm{~T}_{h^{n}} a\left(x, h^{n}\right)=-\mathrm{P}_{h^{n}} \mathrm{~F}_{h^{n}} a\left(x, h^{n}\right)+\varepsilon\left(x, h^{n}\right),
$$

г де $\varepsilon\left(x, h^{n}\right) \rightarrow 0$ по норме $M\left(-r, h^{n}, \Omega_{1 h^{n}}\right)$ при $\left|h^{n}\right| \rightarrow 0$.

Будем рассматривать (2.15) как уравнение относительно функции $a\left(x, h^{n}\right)$, находящейся в левой части, а правую часть будем считать известной. Из предложения 6.5 (см. [2]) следует, что $\mathrm{P}_{1 h^{n} \varepsilon}\left(x, h^{n}\right) \rightarrow 0$ и $b\left(x, h^{n}\right) \rightarrow b(x)$ по норме $H_{-r}(\Omega)$. Тогда по следствию 2.1 функции $a(x, h)$ слабо сходятся к функции $u(x) \in \stackrel{\circ}{H}_{r}(\Omega)$, являюшейся решением уравнения $\mathrm{PT} u(x)=-b(x), u(x) \neq 0$. Докажем, что $\mathrm{PA} u(x)=0$. Для этого достаточно убедиться в том, что $\mathrm{PF} u(x)=b(x)$ или, что то же самое, $(\nu, \mathrm{F} u(x))=(\nu, b(x))$ для любой $\nu(x) \in \stackrel{\circ}{H}_{r}(\Omega)$.

Согласно замечанию 1.2 (см. предложение 1.7) $\mathrm{PF} u(x)$, сужение линейного функционала $\mathrm{F} u(x)$ на $\stackrel{\circ}{H}_{r}(\Omega)$, имеет представление

$$
\operatorname{PF} u(x)=\mathrm{P}_{1 h^{n}} \mathrm{~F}_{h^{n}} \prod_{1 h^{n}} u(x)+\varepsilon_{1}\left(x, h^{n}\right),
$$

где $\varepsilon_{1}\left(x, h^{n}\right) \rightarrow 0$ по норме $H_{-r}(\Omega)$ при $\left|h^{n}\right| \rightarrow 0$. 
Используя (2.16), получаем соотношение

$$
\begin{aligned}
(\nu, \mathrm{F} u(x))= & \lim _{\left|h^{n}\right| \rightarrow 0}\left(\nu(x), \mathrm{P}_{1 h^{n}} \mathrm{~F}_{h^{n}} \prod_{1 h^{n}} u(x)\right) \\
= & \lim _{\left|h^{n}\right| \rightarrow 0}\left(\nu(x), \mathrm{P}_{1 h^{n}} \mathrm{~F}_{h^{n}} a\left(x, h^{n}\right)\right) \\
& \quad+\lim _{\left|h^{n}\right| \rightarrow 0}\left(\nu(x), \mathrm{P}_{1 h^{n}} \mathrm{~F}_{h^{n}}\left(\prod_{1 h^{n}} u(x)-a\left(x, h^{n}\right)\right)\right) \\
= & (\nu(x), b(x))+\lim _{\left|h^{n}\right| \rightarrow 0}\left(\prod_{1 h^{n}} \nu(x), \mathrm{P}_{1 h^{n}} \mathrm{~F}_{h^{n}}\left(\prod_{1 h^{n}} u(x)-a\left(x, h^{n}\right)\right)\right) \\
= & (\nu(x), b(x))+\lim _{\left|h^{n}\right| \rightarrow 0} I\left(h^{n}\right) .
\end{aligned}
$$

Так как семейство операторов $\mathrm{F}_{h}$ ограничено из $\stackrel{\circ}{M}\left(s, h, \Omega_{1 h}\right)$ в $M(-r+\varepsilon, h)$, $\varepsilon>0$, для любого $s$, удовлетворяющего неравенству $0<r_{0} \leqslant s<r$, то имеет место неравенство

$$
\begin{aligned}
& \left|I\left(h^{n}\right)\right| \leqslant\left\|\prod_{1 h^{n}} \nu(x)\right\|_{\stackrel{\circ}{M}\left(r, h^{n}, \Omega_{1 h^{n}}\right)} \\
& \times\left\|\mathrm{P}_{h^{n}} \mathrm{~F}_{h^{n}}\left(\prod_{1 h^{n}} u(x)-a\left(x, h^{n}\right)\right)\right\|_{M\left(-r, h^{n}, \Omega_{1 h^{n}}\right)} \\
& \leqslant\left\|\prod_{1 h^{n}} \nu(x)\right\|_{\stackrel{\circ}{M\left(r, h^{n}, \Omega_{1 h^{n}}\right)}} \\
& \times\left\|\mathrm{F}_{h^{n}}\left(\prod_{1 h^{n}} u(x)-a\left(x, h^{n}\right)\right)\right\|_{M\left(-r, h^{n}\right)} \\
& \leqslant C\left\|\prod_{1 h^{n}} \nu(x)\right\|_{\stackrel{\circ}{\circ}\left(r, h^{n}, \Omega_{1 h^{n}}\right)} \\
& \times\left\|\mathrm{F}_{h^{n}}\left(\prod_{1 h^{n}} u(x)-a\left(x, h^{n}\right)\right)\right\|_{M\left(-r+\varepsilon, h^{n}\right)} \\
& \leqslant C_{1}\left\|\mathrm{~F}_{h^{n}}\right\|_{s_{0},-r+\varepsilon} \\
& \times\left\|\prod_{1 h^{n}} u(x)-a\left(x, h^{n}\right)\right\|_{\stackrel{\circ}{M\left(s_{0}, h^{n}, \Omega_{1 h^{n}}\right)}}\|\nu(x)\|_{\stackrel{\circ}{\circ}(\Omega)},
\end{aligned}
$$

$r_{0} \leqslant s_{0}<r,\left\|\mathrm{~F}_{h^{n}}\right\|_{s_{0},-r+\varepsilon}-$ обозначение нормы оператора $\mathrm{F}_{h}$ из $\stackrel{\circ}{M}\left(s_{0}, h^{n}, \Omega_{1 h^{n}}\right)$ в $M\left(-r+\varepsilon, h^{n}\right)$. Функции $a\left(x, h^{n}\right)$ слабо сходятся к $u(x) \in \stackrel{\circ}{H}_{r}(\Omega)$ (см. определение 2.3), поэтому из (2.18) вытекает $\lim _{\left|h^{n}\right| \rightarrow 0} I\left(h^{n}\right)=0$. Отсюда и из соотношения (2.17) следует равенство РА $u(x)=0, u(x) \neq 0$, что противоречит условиям предложения 2.5. Итак, предложение 2.5 доказано.

\section{§ 3. Ограниченные семейства операторов в пространствах дробных отношений $M(r, h)$}

3.1. Одномерный дискретный сингулярный оператор. Рассмотрим разбиение числовой оси на интервалы $D(k, h)=\left(h\left(k-\frac{1}{2}\right), h\left(k+\frac{1}{2}\right)\right)$ с шагом $h>0, k \in \mathbb{Z}$. Пусть семейство операторов $\mathrm{T}_{h}$, определенных на функциях $u(x, h) \in M(r, h)$, задается соотношением:

$$
\pi \mathrm{T}_{h} u(x, h)=\lim _{N \rightarrow \infty} \sum_{m=-N}^{N} \gamma(m) u(k-m)=\sum_{m=-\infty}^{\infty} \gamma(m) u(k-m),
$$


где оператор $\pi$ введен в [2; определение 1.2$], u(k)=\pi u(x, h)$, числа $\gamma(m)$ определяются равенством

$$
\gamma(m)=\int_{(m-1 / 2) h}^{(m+1 / 2) h} \frac{d x}{x}=\int_{(m-1 / 2) h}^{(m+1 / 2) h} \frac{d y}{y} .
$$

Из (3.2) следует, что $\gamma(0)=0, \gamma(-m)=-\gamma(m)$. Используя (1.9) из [2], получаем:

$$
\pi \mathrm{T} u(x, h)=\frac{1}{2 \pi} \int_{-\pi}^{\pi} S_{1}(\varphi) \widehat{u}_{c}(\varphi) e^{-i k \varphi} d \varphi
$$

где $\widehat{u}_{c}(\varphi)$ - дискретное преобразование Фурье (см. [2; определение 1.4]) сеточной функции $u(k)=\pi a(x, h)$, функция $S_{1}(\varphi)$ задается соотношением

$$
S_{1}(\varphi)=\lim _{N \rightarrow \infty} S_{1}(N, \varphi)=\lim _{N \rightarrow \infty} \sum_{k=-N}^{N} \gamma(k) e^{i k \varphi} .
$$

Из (3.4) и нечетности $\gamma(k)$ вытекает равенство

$$
S_{1}(\varphi)=2 i \sum_{k=1}^{\infty} \gamma(k) \sin k \varphi
$$

Отметим, что из определения $\gamma(k)$ следуют неравенства

$$
|\gamma(k)|<\frac{C}{k} ; \quad|\gamma(k)-\gamma(k+1)|<\frac{C}{k^{2}} .
$$

Кроме того, для ядра $D_{n}(x)=\sum_{\nu=1}^{n} \sin \nu x=\frac{\cos \frac{x}{2}-\cos \left(n+\frac{1}{2}\right) x}{2 \sin \frac{x}{2}}$ вьполняются оценки

$$
\left|D_{n}(x)\right| \leqslant n, \quad\left|D_{n}(x)\right| \leqslant \frac{1}{\sin \frac{x}{2}} .
$$

Докажем, что функция $S_{1}(\varphi)$ ограничена на $[-\pi, \pi]$.

Возьмем некоторое $\delta>0$ (пусть $\delta=\frac{1}{100}$ ). Рассмотрим произвольное $\varphi,|\varphi| \geqslant \delta$. Тогда, используя преобразование Абеля, получаем

$$
\begin{aligned}
\left|S_{1}(N, \varphi)\right| & =\left|2 \sum_{k=1}^{N} \gamma(k) \sin k \varphi\right| \\
& \leqslant 2 \sum_{k=1}^{N-1}|\gamma(k)-\gamma(k+1)|\left|\sum_{l=1}^{k} \sin l \varphi\right|+|\gamma(N)|\left|\sum_{l=1}^{N} \sin l \varphi\right| \\
& \leqslant C \sum_{k=1}^{N-1} \frac{1}{k^{2}} \frac{1}{\sin \frac{\delta}{2}}+\frac{C}{N \sin \frac{\delta}{2}} \leqslant \frac{C_{1}}{\sin \frac{\delta}{2}} .
\end{aligned}
$$

Пусть $\varphi$ удовлетворяет неравенству $|\varphi|<\delta ; S_{1}(\varphi)=0$ при $\varphi=0$, поэтому можно считать, что $\varphi \neq 0$. Множества $K(N)=\{\varphi: 1 /(2 N)<|\varphi| \leqslant 1 / N\}$ покрьвают 
множество $K=\{\varphi: 0<|\varphi| \leqslant 1\}$. Поэтому для любого фиксированного $\varphi \in K$ найдется такое $N$, что $\varphi \in K(N)$. Пусть $\varphi \in K(N)$.

Рассмотрим функцию

$$
\begin{aligned}
S_{1}(p, \varphi)=2 i \sum_{k=1}^{p} \gamma(k) \sin k \varphi & =2 i \sum_{k=1}^{N-1} \gamma(k) \sin k \varphi+2 i \sum_{k=N}^{p} \gamma(k) \sin k \varphi \\
& =S_{1}(N-1, \varphi)+S_{2}(N, p, \varphi) .
\end{aligned}
$$

Для слагаемого $S_{1}(N-1, \varphi)$ получим оценку

$$
\left|S_{1}(N-1, \varphi)\right| \leqslant 2 \sum_{k=1}^{N-1}|\gamma(k)| \cdot k|\varphi| \leqslant 2 C N \varphi \leqslant 2 C .
$$

Используя преобразование Абеля, получаем

$$
\begin{aligned}
\left|S_{2}(N, p, \varphi)\right|= & \left|2 \sum_{k=N}^{p} \gamma(k) \sin k \varphi+\gamma(N) \sum_{k=1}^{N-1} \sin k \varphi-\gamma(N) \sum_{k=1}^{N-1} \sin k \varphi\right| \\
\leqslant & 2 \sum_{k=N}^{p-1}|\gamma(k)-\gamma(k+1)|\left|\sum_{l=1}^{k} \sin l \varphi\right| \\
& +2|\gamma(p)|\left|\sum_{k=1}^{p} \sin k \varphi\right|+2|\gamma(N)|\left|\sum_{k=1}^{N-1} \sin k \varphi\right| \\
\leqslant & C_{1} \sum_{k=N}^{p-1} \frac{1}{k^{2} \sin \frac{|\varphi|}{2}}+\frac{C_{1}}{p \sin \frac{|\varphi|}{2}}+\frac{C_{1}}{N \sin \frac{|\varphi|}{2}} .
\end{aligned}
$$

Так как $|\varphi| / 2 \in K(N)$, то из (3.9) вытекает неравенство

$$
\left|S_{2}(N, p, \varphi)\right| \leqslant C_{2} .
$$

Из неравенства (3.9) следует, что ряд $2 i \sum_{k=1}^{\infty} \gamma(k) \sin k \varphi$ сходится при любом $\varphi \neq 0$, а так как он заведомо сходится при $\varphi=0$, то этот ряд сходится для любого $\varphi \in[-\pi, \pi]$. Из оценок $(3.7),(3.8)$ и $(3.10)$ следует ограниченность $S_{1}(\varphi)$ на $[-\pi, \pi]$.

ОпрЕДЕЛЕнИЕ 3.1. Семейство операторов $\mathrm{T}_{h}$, определенных по формуле (3.1), будем называть семейством дискретных одномерных сингулярных операторов.

ПРЕДЛОЖЕНИЕ 3.1. Семейство одномерных дискретных сингулярных операторов $\mathrm{T}_{h}$ равномерно ограниченно отображает пространства $M(r, h)$ в $M(r, h)$, где $r$ - любое число.

ДокаЗАТЕЛЬСтво. Функция $S_{1}(\varphi)$ (символ операторов $\mathrm{T}_{h}$ ) не зависит от $h$ и ограничена на $[-\pi, \pi]$. При каждом фиксированном $h$ функция $u(x, h) \in M(r, h)$ принадлежит также $L_{2}\left(\mathbb{R}_{1}\right)$. Поэтому $S_{1}(\varphi) \widehat{u}_{c}(\varphi) \in L_{2}[-\pi, \pi]$. Тогда из формулы (3.3) имеем $\mathrm{T}_{h} u(x, h) \in L_{2}\left(\mathbb{R}_{1}\right)$. Отсюда следует, что существует функция $\widehat{a}_{c}(\varphi)$ - дискретное преобразование Фурье сеточной функции $a(k)=\pi \mathrm{T}_{h} u(x, h)$ (см. [2; определение 1.4]), для которой выполняется $\widehat{a}_{c}(\varphi)=S_{1}(\varphi) \cdot \widehat{u}_{c}(\varphi)$. Из последнего замечания и определения нормы в $M(r, h)$ следует предложение 3.1 . 
3.2. Многомерный дискретный сингулярный оператор. Рассмотрим сингулярный интеграл в $\mathbb{R}_{n}$ :

$$
\int \frac{f(\vec{\theta}) \nu(x) d x}{r^{n}}
$$

где $r=r_{M M_{0}}$ - расстояние между точками $M\left(x_{1}, \ldots, x_{n}\right)$ и $M_{0}\left(x_{1}^{0}, \ldots, x_{n}^{0}\right), f(\vec{\theta})$ характеристика сингулярного интеграла, являюшегося функцией вектора $\vec{\theta}=\frac{\mathbf{r}}{r}$, $f(\vec{\theta})$ удовлетворяет условию Липшица, а также

$$
\int_{S_{n}} f(\vec{\theta}) d S_{n}=0
$$

где $S_{n}$ - единичная сфера в $\mathbb{R}_{n}$.

Рассмотрим каноническое разбиение пространства $\mathbb{R}_{n}$ с векторным шагом $h=(h, \ldots, h)$ (т.е. шаг дробления $h$ на каждой оси одинаков) на ячейки $D(k, h)$ (см. [2; определение 1.1]). Пусть семейство операторов $\mathrm{T}_{h}$, определенных на функциях $u(x, h) \in M(r, h)$, задается соотношением

$$
\begin{aligned}
\pi \mathrm{T}_{h} u(x, h) & =\lim _{N \rightarrow \infty} \sum_{m_{1}=-N}^{N} \cdots \sum_{m_{n}=-N}^{N} \gamma\left(m_{1}, \ldots, m_{n}\right) u\left(k_{1}-m_{1}, \ldots, k_{n}-m_{n}\right) \\
& =\sum_{m \in \mathbb{Z}_{n}} \gamma(m) u(k-m)
\end{aligned}
$$

где оператор $\pi$ вводится в [2; определение 1.2], $u(k)=\pi \mathrm{T}_{h} u(x, h)$, числа $\gamma(m)$ определяются равенством

$$
\gamma(m)=\int_{\left(m_{1}-1 / 2\right) h}^{\left(m_{1}+1 / 2\right) h} \cdots \int_{\left(m_{n}-1 / 2\right) h}^{\left(m_{n}+1 / 2\right) h} \frac{f(\vec{\theta})}{r^{n}} d x,
$$

где $r=\sqrt{\sum_{j=1}^{n} x_{j}^{2}}$.

Сделаем в (3.13) замену $x_{j}=h y_{j}, j=1, \ldots, n$. Так как при данном преобразовании вектор $\vec{\theta}=\frac{\mathbf{r}}{r}$ не меняется, то из (3.13) получим:

$$
\gamma(m)=\int_{D(m, 1)} \frac{f(\vec{\theta})}{r^{n}} d x .
$$

Из (3.14) следует, что $\gamma(m)$ не зависит от $h$, сеточная функция $\gamma(m) \in l_{2}$. Отсюда вытекает, что символ операторов $\mathrm{T}_{h}$ функция $S_{n}\left(\varphi_{1}, \ldots, \varphi_{j}\right) \in L_{2}(U)$, $U=\prod_{j=1}^{n}\left[-\pi \leqslant \varphi_{j} \leqslant \pi\right]$. Символ $S_{n}(\varphi)$ задается соотношением (см. [2; определение 1.6])

$$
S_{n}(\varphi)=\lim _{N \rightarrow \infty} \sum_{k \in \mathbb{Z}_{n}(N)} a(k) e^{i(k, \varphi)} .
$$

Отсюда аналогично (3.3) получим

$$
\pi \mathrm{T}_{h} u(x, h)=\frac{1}{(2 \pi)^{n}} \int_{U} S_{n}(\varphi) \widehat{u}_{c}(\varphi) e^{-i(k, \varphi)} d \varphi
$$


где $(k, \varphi)=\sum_{l=1}^{n} \varphi_{l} k_{l}$.

Аналогично (3.14) запишем

$$
\Gamma(N)=\sum_{k \in \mathbb{Z}_{n}(N)} \gamma(k)=\int_{D(0,1)} \frac{f(\vec{\theta}) d x}{r^{n}}=\gamma(0)
$$

Введем величины

$$
\Gamma_{l}(m)=\sum_{\substack{i=1 \\ i \neq l}}^{n} \sum_{k_{i}=-\infty}^{\infty}\left|\gamma\left(k_{1}, \ldots, k_{l-1}, k_{l}=m, k_{l+1}, \ldots, k_{n}\right)\right| .
$$

Оценим $\Gamma_{1}(0)$ :

$$
\begin{aligned}
\Gamma_{1}(0) & \leqslant|\gamma(0)|+\sum_{k_{2}=-\infty}^{\infty} \ldots \sum_{k_{n}=-\infty}^{\infty}\left|\gamma\left(0, k_{2}, \ldots, k_{n}\right)\right| \\
& \leqslant C+\int_{-1 / 2}^{1 / 2} d x_{1} \int_{\Omega_{n-1}(1 / 2)} \frac{|f(\vec{\theta})|}{r^{n}} d x_{2} \cdots d x_{n} \\
& \leqslant C+C_{1} \int_{-1 / 2}^{1 / 2} d x_{1} \int_{S_{n-1}}\left\{\int_{1 / 2}^{\infty} \frac{d r_{1}}{r_{1}^{2}}\right\} d S_{n-1} \leqslant C_{2},
\end{aligned}
$$

где $|k| \neq 0, \Omega_{n-1}(1 / 2)$ - внешность шара $(n-1)$-мерного пространства радиуса $R=\frac{1}{2}, S_{n-1}-$ поверхность единичной сферы этого пространства, $r_{1}^{2}=\sum_{i=2}^{n} x_{i}^{2}$.

Для получения оценок величин $\Gamma_{1}(m), m>0$, введем сферические координаты так же, как и при выводе соотношения (3.7) в [2]. Далее получим:

$$
\begin{aligned}
\Gamma_{1}(m) & \leqslant C \int_{S_{n-1}} d S_{n-1} \int_{-\pi / 2}^{\pi / 2} \sin ^{n-2} \Theta_{1} d \Theta_{1} \int_{\frac{m-1 / 2}{\cos \Theta_{1}}}^{\frac{m-1 / 2}{\cos \Theta_{1}}} \frac{d r}{r} \\
& \leqslant C_{1} \ln \left(1+\frac{2}{2 m-1}\right) \leqslant \frac{C_{2}}{|m|+1} .
\end{aligned}
$$

Аналогично получается оценка $\Gamma_{l}(m)$ для любых $l$ и $m$ :

$$
\Gamma_{l}(m)<\frac{C}{|m|+1}
$$

Перейдем теперь к оценкам величин

$$
\Delta \Gamma_{l}(m)=\sum_{\substack{i=1 \\ i \neq l}}^{n} \sum_{k_{i}=-\infty}^{\infty}\left|\gamma\left(k_{1}, \ldots, k_{l}=m, \ldots, k_{n}\right)-\gamma\left(k_{1}, \ldots, k_{l}=m+1, \ldots, k_{n}\right)\right| .
$$

Используя условие Липшица на функцию $f(\vec{\theta})$, а также выполняя элементарные преобразования, получаем при $m \neq 0, m+1 \neq 0$ оценку

$$
\left|\gamma\left(m, k_{2}, \ldots, k_{n}\right)-\gamma\left(m+1, k_{2}, \ldots, k_{n}\right)\right|<C \int_{D\left(m, k_{2}, \ldots, k_{n}, 1\right)} \frac{d x}{r^{n+1}} .
$$


Отсюда, введя, как и ранее, сферические координаты, получим при $m>0$

$$
\Delta \Gamma_{1}(m) \leqslant C_{1} \int_{S_{n-1}} d S_{n-1} \int_{-\pi / 2}^{\pi / 2} \sin ^{n-2} \Theta_{1} d \Theta_{1} \int_{\frac{m-1 / 2}{\cos \Theta_{1}}}^{\frac{m+1 / 2}{\cos \Theta_{1}}} \frac{d r}{r^{2}} \leqslant \frac{C_{2}}{m^{2}+1} .
$$

Аналогичньм образом выводим оценку для любых $l=1, \ldots, n$ и любых $m \in \mathbb{Z}$ :

$$
\Delta \Gamma_{l}(m) \leqslant \frac{C_{2}}{m^{2}+1}
$$

Перейдем к оценкам величин

$$
\begin{aligned}
& \Delta(l) \Gamma\left(k_{j}=N\right)=\sum_{k_{j}=N+1}^{+\infty} \sum_{\substack{p=1 \\
p \neq j}}^{n} \sum_{k_{p}=-\infty}^{\infty} \mid \gamma\left(k_{1}, \ldots, k_{l}, \ldots, k_{n}\right) \\
& -\gamma\left(k_{1}, \ldots, k_{l}+1, \ldots, k_{n}\right) \mid, \\
& \Delta(l) \Gamma\left(k_{j}=-N\right)=\sum_{j=-\infty} \sum_{\substack{p=1 \\
p \neq j}}^{n} \sum_{k_{p}=-\infty}^{\infty} \mid \gamma\left(k_{1}, \ldots, k_{l}, \ldots, k_{n}\right) \\
& -\gamma\left(k_{1}, \ldots, k_{l}+1, \ldots, k_{n}\right) \mid,
\end{aligned}
$$

где $l \neq j, N>0, N$ - целое.

Используя оценку (3.23), получаем:

$$
\Delta(l) \Gamma\left(k_{1}=N\right) \leqslant C \int_{S_{n-1}} d S_{n-1} \int_{-\pi / 2}^{\pi / 2} \sin ^{n-2} \Theta_{1}\left(\int_{\frac{N+1}{\cos \Theta_{1}}}^{\infty} \frac{d r}{r^{2}}\right) d \Theta_{1} \leqslant \frac{C}{N+1 / 2} .
$$

Аналогично выводятся оценки для всех $l=1, \ldots, n$ и всех $k_{j}=N, k_{j}=-N$, $l \neq j$ :

$$
\Delta(l) \Gamma\left(k_{j}=N\right) \leqslant \frac{C}{N+1} ; \quad \Delta(l) \Gamma\left(k_{j}=-N\right) \leqslant \frac{C}{N+1} .
$$

ПРЕДЛОЖЕНИЕ 3.2. Если характеристика сингулярного интеграла удовлетворяет условию Липшица, то функиия

$$
S_{n}(\varphi)=\lim _{N \rightarrow \infty} \sum_{k \in \mathbb{Z}_{n}(N)} \gamma(k) e^{i(k, \varphi)}
$$

является ограниченной функцией при $\varphi \in \prod_{j=1}^{n}\left[-\pi \leqslant \varphi_{j} \leqslant \pi\right]$.

ДокАЗАтЕЛЬСтво. Пусть $|\varphi| \geqslant \delta>0$, возьмем точку $M\left(\varphi_{1}, \ldots, \varphi_{j}\right)$, удовлетворяющую этому условию. Тогда хотя бы одна координата $\varphi_{j}$ удовлетворяет условию $\left|\varphi_{j}\right| \geqslant \delta / \sqrt{n}$. Ввиду симметричности оценок $(3.21),(3.25)$ и (3.27) можно считать, что $\left|\varphi_{1}\right| \geqslant \delta / \sqrt{n}$. 
Рассмотрим функцию

$$
\begin{aligned}
S_{n}(N, \varphi) & =\sum_{k \in \mathbb{Z}_{n}(N)} \gamma(k) e^{i(k, \varphi)} \\
& =\sum_{k_{1}=-N}^{-1} \sum_{p=2}^{n} \sum_{k_{p}=-N}^{N} \gamma(k) e^{i(k, \varphi)}+\sum_{k_{1}=0}^{N} \sum_{p=2}^{n} \sum_{k_{p}=-N}^{N} \gamma(k) e^{i(k, \varphi)} \\
& =S_{n}^{1}(N, \varphi)+S_{n}^{2}(N, \varphi)
\end{aligned}
$$

где $(k, \varphi)=\sum_{j=1}^{n} k_{j} \varphi_{j}, k=\left(k_{1}, \ldots, k_{n}\right), \varphi=\left(\varphi_{1}, \ldots, \varphi_{j}\right)$.

Выполнив преобразование Абеля по переменной $k_{1}$ при каждом фиксированном $k^{\prime}=\left(k_{2}, \ldots, k_{n}\right)$, запишем

$$
\begin{aligned}
S_{n}^{2}(N, \varphi)= & \sum_{k_{1}=0}^{N-1} \sum_{p=2}^{n} \sum_{k_{p}=-N}^{N}\left[\gamma\left(k_{1}, k^{\prime}\right)-\gamma\left(k_{1}+1, k^{\prime}\right)\right] e^{i\left(k^{\prime}, \varphi^{\prime}\right)} \sum_{l=0}^{k_{1}} e^{i l \varphi_{1}} \\
& +\sum_{p=2}^{n} \sum_{k_{p}=-N}^{N} \gamma\left(N, k^{\prime}\right) e^{i\left(k^{\prime}, \varphi^{\prime}\right)} \sum_{l=0}^{N} e^{i l \varphi_{1}}=\Delta_{1}+\Delta_{2} .
\end{aligned}
$$

Используя оценки (3.21) и (3.25), получаем

$$
\begin{aligned}
& \left|\Delta_{1}\right| \leqslant \sum_{m=0}^{N-1} \Delta \Gamma_{1}(m) \frac{2}{\sin \frac{\left|\varphi_{1}\right|}{2}} \leqslant \frac{C_{1}}{\sin \frac{\delta}{2}} \sum_{m=0}^{N-1} \frac{1}{m^{2}+1} \leqslant \frac{C_{1}}{\sin \frac{\delta}{2}} \\
& \left|\Delta_{2}\right| \leqslant \Gamma_{1}(N) \frac{2}{\sin \frac{\delta}{2}} \leqslant \frac{C}{N \sin \frac{\delta}{2}} .
\end{aligned}
$$

С функцией $S_{n}^{1}(n, \varphi)$ поступим аналогично, сделав для удобства замену индекса суммирования $k_{1}=-l, 1 \leqslant l \leqslant N$ :

$$
\begin{aligned}
S_{n}^{1}(N, \varphi)= & \sum_{l=1}^{N-1} \sum_{p=2}^{n} \sum_{k_{p}=-N}^{N}\left[\gamma\left(-l, k^{\prime}\right)-\gamma\left(-l-1, k^{\prime}\right)\right] e^{i\left(k^{\prime}, \varphi^{\prime}\right)} \sum_{l_{1}=1}^{l} e^{-i \varphi_{1} l_{1}} \\
& +\sum_{p=2}^{n} \sum_{k_{p}=-N}^{N} \gamma\left(-N, k^{\prime}\right) \sum_{l_{1}=1}^{N} e^{-i \varphi_{1} l_{1}}=\Delta_{1}^{\prime}+\Delta_{2}^{\prime}
\end{aligned}
$$

Равенство (3.31) аналогично соотношению (3.29), поэтому имеет место неравенство

$$
\left|S_{n}^{1}(N, \varphi)\right| \leqslant \frac{C}{\sin \frac{\delta}{2}} .
$$

Из (3.28)-(3.32) следует оценка при $|\varphi|>\delta, \varphi \in U=\prod_{j=1}^{n}\left[-\pi \leqslant \varphi_{j} \leqslant \pi\right]$ :

$$
S_{n}(N, \varphi) \leqslant \frac{C_{3}}{\sin \frac{\delta}{2}}
$$


Пусть $\varphi$ удовлетворяет неравенству $|\varphi|<\delta<1$. При $\varphi=0$ имеем $S_{n}(N, 0)=$ $\Gamma(N)=\gamma(0)$, отсюда $S_{n}(0)=\lim _{N \rightarrow \infty} S_{n}(N, 0)=\gamma(0)$. Поэтому можно считать, что $|\varphi| \neq 0$. Множества $K(N)=\{\varphi: 1 /(2 N)<|\varphi| \leqslant 1 / N\}$ покрывают множество $K=\{\varphi: 0<|\varphi| \leqslant 1\}$. Следовательно, для любого фиксированного $\varphi \in K$ найдется такое $N$, что $\varphi \in K(N)$. Тогда хотя бы для одной координаты $\varphi_{j}$ вьполняется неравенство

$$
\frac{1}{2 N \sqrt{n}}<\left|\varphi_{j}\right|<\frac{1}{N}
$$

Без ограничения обшности, как и вьше, можно считать, что этому неравенству удовлетворяет координата $\varphi_{1}$.

Представим функцию $S_{n}(q, \varphi), q>N$, в виде

$$
S_{n}(q, \varphi)=S_{n}(N, \varphi)+\widetilde{S}_{n}(q, \varphi),
$$

где в $\widetilde{S}_{n}(q, \varphi)$ входят все члены, не попавшие в $S_{n}(N, \varphi)$.

Рассмотрим $S_{n}(N, \varphi)$, учитывая (3.17):

$$
\begin{aligned}
\left|S_{n}(N, \varphi)\right| & \leqslant\left|\sum_{k \in \mathbb{Z}_{n}(N)}^{\prime} \gamma(k)\left(e^{i(k, \varphi)}-1\right)\right|+|\gamma(0)| \leqslant \sum_{k \in \mathbb{Z}_{n}(N)}^{\prime}|\gamma(k)| \cdot|k| \cdot|\varphi|+C \\
& \leqslant C\left(|\varphi| \int_{S_{n}} d S_{n} \int_{1 / 2}^{\sqrt{n}(N+1 / 2)} d r+1\right) \leqslant C\left(C_{1} N \cdot|\varphi|+1\right) \leqslant C_{2}, \quad(3
\end{aligned}
$$

где $\sum^{\prime}$ указьвает, что нет члена при $|k|=0, S_{n}$ - единичная сфера в $\mathbb{R}_{n}$.

Разобьем интервал изменения индекса $k_{j},-q \leqslant k_{j} \leqslant q$, на интервалы $I_{1}, I_{2}, I_{3}$ : $I_{1}=\left\{k_{j}:-q \leqslant k_{j} \leqslant-N-1\right\}, I_{2}=\left\{k_{j}:-N \leqslant k_{j} \leqslant N\right\}, I_{3}=\left\{k_{j}: N+1 \leqslant k_{j} \leqslant q\right\}$. Пусть символ $\alpha_{j}$ принимает три значения $1,2,3$, тогда $\sum_{k_{j} \in I_{\alpha_{j}}}$ означает, что суммирование происходит по всем индексам $k_{j} \in I_{\alpha_{j}}$.

Введем обозначение:

$$
S_{\alpha_{1}, \ldots, \alpha_{n}}(\varphi)=\sum_{k_{1} \in I_{\alpha_{1}}} \cdots \sum_{k_{n} \in I_{\alpha_{n}}} \gamma\left(k_{1}, \ldots, k_{n}\right) e^{i(k, \varphi)}
$$

например, $S_{2, \ldots, 2}(\varphi)=S_{n}(N, \varphi)$.

В этих обозначениях функция $\widetilde{S}_{n}(q, \varphi)$ из $(3.34)$ запишется в виде

$$
\widetilde{S}_{n}(q, \varphi)=\sum_{\alpha}^{\prime} S_{a_{1}, \ldots, \alpha_{n}}(\varphi)
$$

где $\sum_{\alpha}^{\prime}$ означает суммирование по всевозможным наборам $\left(\alpha_{1}, \ldots, \alpha_{n}\right)$, причем набор $(2, \ldots, 2)$ пропускается.

Соотношение (3.36) справедливо, так как для любого $k=\left(k_{1}, \ldots, k_{n}\right)$ каждый индекс $k_{j}$ попадает в один из интервалов $I_{1}, I_{2}, I_{3}$.

Поэтому член $\gamma\left(k_{1}, \ldots, k_{n}\right) e^{i(k, \varphi)}$ обязательно появится хотя бы в одной функции $S_{\alpha_{1}, \ldots, \alpha_{n}}(\varphi)$, и ни один такой член не может присутствовать в двух разных функциях, так как интервалы $I_{1}, I_{2}, I_{3}$ не пересекаются. 


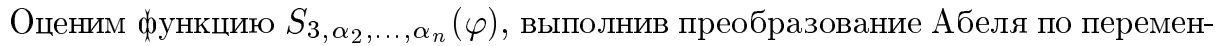
ной $k_{1}$ при каждом фиксированном наборе $k^{\prime}=\left(k_{2}, \ldots, k_{n}\right)$ :

$$
\begin{aligned}
\left|S_{3, \alpha_{1}, \ldots, \alpha_{n}}(\varphi)\right| \leqslant \mid & \sum_{k_{1}=N+1}^{q} \sum_{k^{\prime} \in\left(\alpha_{2}, \ldots, \alpha_{n}\right)}\left[\gamma\left(k_{1}, k^{\prime}\right)-\gamma\left(k_{1}+1, k^{\prime}\right)\right] \\
& \times e^{i\left(k^{\prime}, \varphi^{\prime}\right)} \sum_{l=N+1}^{k_{1}} e^{i l \varphi_{1}} \mid \\
& +\left|\sum_{k^{\prime} \in\left(\alpha_{2}, \ldots, \alpha_{n}\right)} \gamma\left(q, k^{\prime}\right) e^{i\left(k^{\prime}, \varphi^{\prime}\right)} \sum_{l=N+1}^{q} e^{i l \varphi_{1}}\right| \\
\leqslant & \frac{C_{1}}{\sin \frac{\left|\varphi_{1}\right|}{2}}\left(\frac{1}{N+1}+\frac{1}{q+1}\right) .
\end{aligned}
$$

При выводе (3.37) были использованы оценки (3.21) и (3.25).

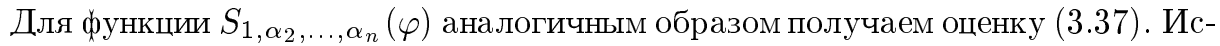
пользуя преобразование Абеля, выписываем соотношение для произвольной функции $S_{2, \alpha_{2}, \ldots, \alpha_{n}}(\varphi)$, входящей в $\widetilde{S}_{n}(q, \varphi)$ :

$$
\begin{aligned}
S_{2, \alpha_{2}, \ldots, \alpha_{n}}(\varphi)= & \sum_{k_{1}=-N}^{N-1} \sum_{k^{\prime} \in\left(\alpha_{2}, \ldots, \alpha_{n}\right)}\left[\gamma\left(k_{1}, k^{\prime}\right)-\gamma\left(k_{1}+1, k^{\prime}\right)\right] \cdot e^{i\left(k^{\prime}, \varphi^{\prime}\right)} \sum_{l=-N}^{k_{1}} e^{i l \varphi_{1}} \\
& +\sum_{k^{\prime} \in\left(\alpha_{2}, \ldots, \alpha_{n}\right)} \gamma\left(N, k^{\prime}\right) e^{i\left(k^{\prime}, \varphi^{\prime}\right)} \sum_{l=-N}^{N} e^{i l \varphi_{1}} .
\end{aligned}
$$

Так как в этом случае среди символов $\alpha_{j}, 2 \leqslant j \leqslant n$, обязательно встретится такой, что либо $\alpha_{j}=1$, либо $\alpha_{j}=3$, то из (3.27) получим

$$
\sum_{k_{1}=-N}^{N-1} \sum_{k^{\prime} \in\left(\alpha_{2}, \ldots, \alpha_{n}\right)}\left|\gamma\left(k_{1}, k^{\prime}\right)-\gamma\left(k_{1}+1, k^{\prime}\right)\right| \leqslant \frac{C}{N+1} .
$$

Из (3.21), (3.38) и (3.39) следует неравенство

$$
\left|S_{2, \alpha_{2}, \ldots, \alpha_{n}}(\varphi)\right| \leqslant \frac{C}{\sin \frac{\left|\varphi_{1}\right|}{2}} \cdot \frac{1}{N+1} .
$$

Из неравенств (3.37) и (3.40) получим оценку

$$
\left|\widetilde{S}_{n}(q, \varphi)\right| \leqslant \frac{C_{2}}{|\varphi|}\left(\frac{1}{N}+\frac{1}{q}\right) .
$$

Из (3.41) вытекает, что сушествует $\lim _{q \rightarrow \infty} S_{n}(q, \varphi)$ для любого $\varphi \in U=$ $\prod_{j=1}^{n}\left[-\pi \leqslant \varphi_{j} \leqslant \pi\right]$. Так как неравенство (3.41) выполняется равномерно при $|\varphi|>\delta>0$, то функция $S_{n}(\varphi)=\lim _{q \rightarrow \infty} S_{n}(q, \varphi)$ будет непрерывна при $|\varphi| \neq 0$. Кроме того, в случае $1 /(2 N)<|\varphi| \leqslant 1 / N$ из (3.41) получим оценку

$$
\left|\widetilde{S}_{n}(q, \varphi)\right| \leqslant C_{0} .
$$

Из (3.35) и (3.42) вытекает, что $\left|S_{n}(q, \varphi)\right| \leqslant B_{0}$ при $0 \leqslant|\varphi|<1$. Из последнего неравенства и (3.33) следует предложение 3.2 . 
ОПРЕДЕЛЕНИЕ 3.2. Семейство операторов $\mathrm{T}_{h}$, определенных по формуле (3.12), называется семейством дискретных сингулярных операторов.

ПРЕДЛОЖЕНИЕ 3.3. Семейство дискретных сингулярных операторов $\mathrm{T}_{h}$ равномерно ограниченно отображсает пространства $M(r, h)$ в $M(r, h), r$ любое, $h=\left(h_{1}, h_{1}, \ldots, h_{1}\right)$.

Доказательство аналогично доказательству предложения 3.1.

3.3. Вихревые дискретные операторы. Пусть семейство операторов $\mathrm{T}_{h, \lambda}$, определенных на функциях $u(x, h) \in M(r, h), h=\left(h_{1}, h_{2}, \ldots, h_{n}\right)$, задается соотношением

$$
\begin{aligned}
\pi \mathrm{T}_{h, \lambda} u(x, h) & =\lim _{N \rightarrow \infty} \sum_{m_{1}=-N}^{N} \ldots \sum_{m_{n}=-N}^{N} \gamma\left(m_{1}, \ldots, m_{n}, \lambda\right) u\left(k_{1}-m_{1}, \ldots, k_{n}-m_{n}\right) \\
& =\sum_{m \in \mathbb{Z}_{n}} \gamma(m, \lambda) u(k-m),
\end{aligned}
$$

где $u(k)=\pi u(x, h)$; числа $\gamma(m, \lambda)$ определяются соотношением

$$
\gamma(m, \lambda)=\int_{D(m, h)} \frac{d x}{r^{n+\lambda}}
$$

$0<\lambda<2$, при $|m|=0$ интеграл понимается по Адамару (см. [2; формула (3.9)]).

ОПрЕДЕлЕНИЕ 3.3. Семейство операторов $\mathrm{T}_{h, \lambda}$, определенных по формулам (3.43) и (3.44), будем называть семейством вихревых дискретных операторов.

ПРЕДЛОЖЕНИЕ 3.4. Семейство вихревых дискретных операторов $\mathrm{T}_{h, \lambda}$ равномерно ограниченно отображает пространства $M(r, h)$ в $M(r-\lambda, h)$, $r$ - любое число.

ДоказАтЕльство. Так как ряд $\sum_{k \in \mathbb{Z}_{n}} \gamma(k, \lambda)$ абсолютно сходится при каждом фиксированном $h=\left(h_{1}, h_{2}, \ldots, h_{n}\right)$, то из [2; формула (1.9)] следует, что сеточную функцию $\pi \mathrm{T}_{h, \lambda} u(x, h)$ можно представить в виде

$$
\pi \mathrm{T}_{h, \lambda} u(x, h)=\frac{1}{(2 \pi)^{n}} \int_{U} J(\lambda, \varphi, h) \widehat{u}_{c}(\varphi) e^{-i(k, \varphi)} d \varphi
$$

где $\widehat{u}_{c}(\varphi)$ - дискретное преобразование Фурье сеточной функции $u(k)=\pi u(x, h)$ (см. [2; определение 1.4$])$; функция $J(\lambda, \varphi, h)$ определяется соотношением

$$
J(\lambda, \varphi, h)=\sum_{k \in \mathbb{Z}_{n}} \gamma(k, \lambda) e^{i(k, \varphi)}
$$


Обозначим через $\widehat{a}_{c}(\varphi)$ дискретное преобразование Фурье сеточной функции $\pi \mathrm{T}_{h, \lambda} u(x, h)$; из предыдушего следует, что $\widehat{a}_{c}(\varphi)=J(\lambda, \varphi, h) \cdot \widehat{u}_{c}(\varphi)$. Тогда, используя соотношения $(2.2),(2.3)$ и (3.15) из [2], получаем:

$$
\begin{aligned}
\left\|\pi \mathrm{T}_{h, \lambda} u(x, h)\right\|_{M(r-\lambda, h)}^{2} & \Omega_{h} \int_{-\pi}^{\pi} \ldots \int_{-\pi}^{\pi}\left(\sqrt{\sum_{i=1}^{n}\left(\frac{\varphi_{i}}{h_{i}}\right)^{2}}+1\right)^{2 r-2 \lambda}\left|\widehat{a}_{c}\left(\varphi_{1}, \ldots, \varphi_{j}\right)\right|^{2} d \varphi_{1} \cdots d \varphi_{j} \\
\leqslant & C(\lambda) \Omega_{h} \int_{-\pi}^{\pi} \cdots \int_{-\pi}^{\pi}\left(\sqrt{\sum_{i=1}^{n}\left(\frac{\varphi_{i}}{h_{i}}\right)^{2}}+1\right)^{2 r} \\
& \times \frac{h_{1}^{2 \lambda}}{\left(\sqrt{\varphi_{1}^{2}+\sum_{i=2}^{n}\left(\varphi_{i} h_{1} / h_{i}\right)^{2}}+h_{1}\right)^{2 \lambda}} \frac{|\varphi|^{2 \lambda}}{h_{1}^{2 \lambda}}\left|\widehat{u}_{c}(\varphi)\right|^{2} d \varphi_{1} \cdots d \varphi_{j} \\
\leqslant & C_{1}(\lambda)\|u(x, h)\|_{M(r, h)}^{2},
\end{aligned}
$$

где $\Omega_{h}=\prod_{j=1}^{n} h_{j}$. Из последнего неравенства следует утверждение 3.4 .

3.4. Разностные операторы. а) Рассмотрим правый разностный оператор $\mathrm{T}_{h_{\text {пр }}}^{m}$ порядка $m$. Согласно [2; пример 1.4] его можно представить в виде

$$
\pi \mathrm{T}_{h_{\text {пр }}}^{m} u(x, h)=\frac{1}{(2 \pi)^{n}} \int_{-\pi}^{\pi} \cdots \int_{-\pi}^{\pi} \prod_{j=1}^{n}\left(\frac{e^{-i \varphi_{j}}-1}{h_{j}}\right)^{m_{j}} \widehat{u}_{c}(\varphi) e^{-i(k, \varphi)} d \varphi_{1} \cdots d \varphi_{j}
$$

где $m_{1}+\cdots+m_{n}=m, \widehat{u}_{c}(\varphi)$ - дискретное преобразование Фурье сеточной функции $u(k)=\pi u(x, h)$; оператор $\pi$ задается в [2; определение 1.2$], u(x, h) \in M(r, h)$. Символ $\widehat{A}_{h}(\varphi)$ оператора $\mathrm{T}_{h_{\text {пр }}}^{m}$ определяется соотношением

$$
\widehat{A}_{h}(\varphi)=\prod_{k=1}^{n}\left(\frac{e^{-i \varphi_{k}}-1}{h_{k}}\right)^{m_{k}}
$$

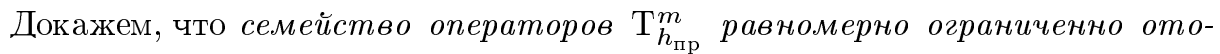
бражсает пространства $M(r, h)$ в $M(r-m, h)$. Действительно, используя (2.2) и (2.3) из [2], получаем

$$
\begin{aligned}
\left\|\mathrm{T}_{h_{\text {пр }}}^{m} u(x, h)\right\|_{M(r-m, h)}^{2}= & \Omega_{h} \int_{-\pi}^{\pi} \ldots \int_{-\pi}^{\pi}\left(\sqrt{\sum_{i=1}^{n}\left(\frac{\varphi_{i}}{h_{i}}\right)^{2}}+1\right)^{2 r-2 m} \\
& \times\left|\prod_{k=1}^{n}\left(\frac{e^{-i \varphi_{k}}-1}{h_{k}}\right)^{m_{k}} \widehat{u}_{c}(\varphi)\right|^{2} d \varphi_{1} \cdots d \varphi_{j} \\
\leqslant & C \Omega_{h} \int_{-\pi}^{\pi} \ldots \int_{-\pi}^{\pi}\left(\sqrt{\sum_{i=1}^{n}\left(\frac{\varphi_{i}}{h_{i}}\right)^{2}}+1\right)^{2 r} \\
& \times \frac{h_{1}^{2 m}}{\left(\sqrt{\varphi_{1}^{2}+\sum_{i=2}^{n}\left(\varphi_{i} / h_{i}\right)^{2}}+1\right)^{2 m}} \frac{|\varphi|^{2 m}}{h_{1}^{2 m}}\left|\widehat{u}_{c}\right|^{2} d \varphi \\
\leqslant & C_{1}\|u(x, h)\|_{M(r, h)}^{2}
\end{aligned}
$$


Из последнего неравенства следует сформулированное выше утверждение.

Аналогичное утверждение справедливо и для разностного смешанного оператора порядка $m$.

б) Итерированный разностный оператор Лапласа $\Delta_{h p}^{m}$. Символ итерированного лапласиана (см. [2; пример 1.5]) имеет вид

$$
\widehat{A}_{c}(\varphi)=(-1)^{m} 4^{m}\left[\sum_{k=1}^{n} \frac{1}{h_{k}^{2}} \sin ^{2} \frac{\varphi_{k}}{2}\right]^{m}
$$

Исходя из (3.49) аналогично (3.48) доказьвается утверждение: семейство операторов $\Delta_{h p}^{m}$ равномерно ограниченно отображсает пространства $M(r, h)$ в $M(r-2 m, h)$.

в) Гиперсингулярные дискретные операторы.

ОПРЕДЕЛЕНИЕ 3.4. Гиперсингулярными дискретными операторами $\mathrm{T}_{h}(m, \lambda)$ будем называть операторы, заданные соотношениями

$$
\mathrm{T}_{h}(m, \lambda)=\mathrm{T}_{h, \lambda}\left(\Delta_{h p}^{m} u(x, h)\right)
$$

где $u(x, h) \in M(r, h), \mathrm{T}_{h, \lambda}-$ вихревой дискретный оператор.

Из предложения 3.4 и п. 3.4.б) следует утверждение: семейство гиперсингулярных дискретных операторов $\mathrm{T}_{h}(m, \lambda)$ равномерно ограниченно отображсает пространства $M(r, h)$ в $M(r-2 m-\lambda, h)$.

\section{§4. Квадратурные формулы}

\section{1. Квадратурные формулы для интегралов вида}

$$
u\left(x_{0}\right)=\int_{\mathbb{R}_{n}} \frac{\varphi(x) d x}{r^{n+\lambda}}, \quad 0<\lambda<2,
$$

где $r=\left|x^{0}-x\right|$, интеграл в (4.1) понимается по Адамару.

Рассмотрим каноническое разбиение пространства $\mathbb{R}_{n}$ с векторньп шагом $h=$ $\left(h_{1}, \ldots, h_{n}\right)$ на ячейки $D(k, h)$ (см. [2; определение 1.1]). В каждой ячейке $D(k, h)$ выберем по точке $M_{0 k}\left(h_{1} k_{1}, \ldots, h_{n} k_{n}\right)$. Пусть $r_{0 k}=\left|M M_{0 k}\right|$, где точка $M$ имеет координаты $x=\left(x_{1}, \ldots, x_{n}\right)$. В дальнейшем будем предполагать $\varphi(x) \in C_{0}^{\infty}\left(\mathbb{R}_{n}\right)$. Для приближенного вычисления интеграла (4.1) в точке $M_{0 m}$ используем квадратурную формулу:

$$
S\left(M_{0 m}\right)=\sum_{k} \varphi\left(M_{0 k}\right) \int_{D(k, h)} \frac{d x}{r_{0 m}^{n+\lambda}}
$$

где суммирование происходит по всевозможным $D(k, h)$.

Оценим величину квадратурной погрешности $\Delta u\left(M_{0 m}\right)=u\left(M_{0 m}\right)-S\left(M_{0 m}\right)$. В сумме (4.2), на самом деле, только конечное число слагаемых, так как $\varphi(x) \in$ $C_{0}^{\infty}\left(\mathbb{R}_{n}\right)$. Обозначим через $\varepsilon(\varphi)$ множество номеров $k=\left(k_{1}, \ldots, k_{n}\right)$ ячеек $D(k, h)$, имеющих непустое пересечение с носителем $\varphi(x)$; тогда

$$
\Delta u\left(M_{0 m}\right)=\sum_{k \in \varepsilon(\varphi)} \int_{D(k, h)} \frac{\varphi(x)-\varphi\left(M_{0 k}\right)}{r_{0 m}^{n+\lambda}} d x
$$


Первый случай. Пусть $M_{0 m} \in \operatorname{supp} \varphi(x)$. Рассмотрим выражение

$L=\sum_{i=1}^{n} \varphi_{x_{i}}^{\prime}\left(M_{0 m}\right)\left(x_{i}-x_{i}^{0 k}\right)=\sum_{i=1}^{n} \varphi_{x_{i}}^{\prime}\left(x_{i}-x_{i}^{0 m}\right)+\sum_{i=1}^{n} \varphi_{x_{i}}^{\prime}\left(x_{i}^{0 m}-x_{i}^{0 k}\right)=L_{1}+L_{2}$,

где $x_{i}^{0 m}$ и $x_{i}^{0 k}, i=1, \ldots, n,-$ координаты точек $M_{0 m}$ и $M_{0 k}$ соответственно. Возьмем шар $K_{R}\left(M_{0 m}\right)$ с центром в $M_{0 m}$ достаточно большого радиуса $R$ так, чтобы ячейки $D(k, h)$, полностью вошедшие в $K_{R}$, покрывали носитель функции $\varphi(x)$. Множество номеров $k$ таких ячеек обозначим через $\varepsilon_{1}$. Докажем, что выполняется равенство

$$
\begin{aligned}
A & =\sum_{k \in \varepsilon_{1}} \int_{D(k, h)} \frac{L(x) d x}{r_{0 m}^{n+\lambda}} \\
& =\sum_{k \in \varepsilon_{1}} \int_{D(k, h)} \frac{L_{1}(x) d x}{r_{0 m}^{n+\lambda}}+\sum_{k \in \varepsilon_{1}} \int_{D(k, h)} \frac{L_{2}(x) d x}{r_{0 m}^{n+\lambda}}=A_{1}+A_{2}=0 .
\end{aligned}
$$

Действительно, если ввести новые переменные $y_{j}=x_{j}-x_{j}^{0 m}, j=1, \ldots, n$, то $A_{1}=0$ как интеграл от нечетной функции по симметричной области (см. определение интеграла по Адамару [2; формула (3.9)]). Так как ячейки $D(k, h)$ расположены симметрично относительно новых осей координат и начала координат, то для любой ячейки $D(k, h)$ найдется такая ячейка $D(\widetilde{k}, h)$, что для всех $j=1, \ldots, n$ будет выполняться $\left(x_{j}^{0 m}-x_{j}^{0 k}\right)=-\left(x_{j}^{0 m}-x_{j}^{0 \widetilde{k}}\right)$. Отсюда $A_{2}=0$.

Разложив $\varphi(x)$ по формуле Тейлора в $M_{0 k}$, получим

$$
\begin{aligned}
\varphi(x)-\varphi\left(M_{0 k}\right)-L(x) & =\sum_{i=1}^{n}\left(x_{i}-x_{i}^{0 k}\right)\left(\varphi_{x_{i}}^{\prime}\left(M_{0 k}\right)-\varphi_{x_{i}}^{\prime}\left(M_{0 m}\right)\right)+O\left(x, M_{0 k}\right) \\
& =B(x)+O\left(x, M_{0 k}\right)
\end{aligned}
$$

где $O\left(x, M_{0 k}\right)$ - остаточный член формулы Тейлора. Значит, $O\left(M_{0 k}, M_{0 k}\right)=0$ и $\left|O\left(x, M_{0 k}\right)\right| \leqslant C|h|^{2}$ при $x \in D(k, h)$.

Ввиду (4.4) и (4.5) равенство (4.3) запишется в виде

$$
\Delta u\left(M_{0 m}\right)=\sum_{k \in \varepsilon_{1}} \int_{D(k, h)} \frac{B(x) d x}{r_{0 m}^{n+\lambda}}+\sum_{k \in \varepsilon_{1}} \int_{D(k, h)} \frac{O\left(x, M_{0 k}\right) d x}{r_{0 m}^{n+\lambda}}=I_{1}+I_{2} .
$$

Произведем оценку каждого слагаемого в (4.6). Оценим сначала $I_{2}$. Пусть $k=m$, тогда $\left|O\left(x, M_{0 m}\right)\right| \leqslant C r_{0 m}^{2}, O\left(M_{0 m}, M_{0 m}\right)=0$. По формуле (3.9) из [2] получим:

$$
\begin{aligned}
& \left|\int_{D(m, h)} \frac{O\left(x, M_{0 m}\right) d x}{r_{0 m}^{n+\lambda}}\right| \\
& \quad=\left|\lim _{\varepsilon \rightarrow 0}\left[\int_{D(m, h) \backslash D(\varepsilon)} \frac{O\left(x, M_{0 m}\right)}{r_{0 m}^{n+\lambda}} d x-\frac{S_{n} O\left(M_{0 m}, M_{0 m}\right)}{\lambda \varepsilon^{\lambda}}\right]\right| \\
& \quad \leqslant C \lim _{\varepsilon \rightarrow 0} \int_{\varepsilon}^{|h| \sqrt{n}} \frac{r_{0 m}^{2}}{r_{0 m}^{n+\lambda}} r_{0 m}^{n-1} d r_{0 m} \leqslant C_{1}(\lambda)|h|^{2-\lambda},
\end{aligned}
$$


где $D(\varepsilon)$ - шар радиуса $\varepsilon>0$ с центром $M_{0 m}, S_{n}$ - плошадь единичной сферы.

Далее рассмотрим

$$
\left|\sum_{\substack{k \in \varepsilon_{1} \\ k \neq m}} \int_{D(k, h)} \frac{O\left(x, M_{0 k}\right) d x}{r_{0 m}^{n+\lambda}}\right| \leqslant C|h|^{2} \int_{|h|}^{\infty} \frac{r_{0 m}^{n-1}}{r_{0 m}^{n+\lambda}} d r_{0 m} \leqslant C(\lambda)|h|^{2-\lambda}
$$

Из (4.7) и (4.8) следует неравенство

$$
\left|I_{2}(h)\right| \leqslant C_{2}(\lambda)|h|^{2-\lambda},
$$

где $C_{3}(\lambda) \rightarrow \infty$ при $\lambda \rightarrow 2$ или $\lambda \rightarrow 0$.

Приступим к оценке $I_{1}$. Пусть $k=m$, тогда $B\left(M_{0 m}\right)=0,|B(x)| \leqslant C r_{0 m}^{2}$ при $x \in D(k, h)$; отсюда, используя формулу (3.9) из [2], получаем

$$
\begin{aligned}
\left|\int_{D(m, h)} \frac{B(x) d x}{r_{0 m}^{n+\lambda}}\right| & =\left|\lim _{\varepsilon \rightarrow 0}\left[\int_{D(m, h) \backslash D(\varepsilon)} \frac{B(x) d x}{r_{0 m}^{n+\lambda}}-\frac{S_{n} B\left(M_{0 m}\right)}{\lambda \varepsilon^{\lambda}}\right]\right| \\
& \leqslant C(\lambda) \lim _{\varepsilon \rightarrow 0} \int_{\varepsilon}^{|h| \sqrt{n}} r_{0 m}^{1-\lambda} d r_{0 m} \leqslant C_{1}(\lambda)|h|^{2-\lambda}
\end{aligned}
$$

Далее рассмотрим

$$
\begin{aligned}
& I_{3}(h)=\left|\sum_{\substack{k \in \varepsilon_{1} \\
k \neq m}} \int_{D(k, h)} \frac{B(x) d x}{r_{0 m}^{n+\lambda}}\right| \\
& \leqslant C(\lambda)|h| \sum_{\substack{k \in \varepsilon_{1} \\
k \neq m}} \int_{D(k, h)} \frac{d x}{r_{0 m}^{n+\lambda-1}} \leqslant C_{1}(\lambda)|h| \int_{|h|}^{R_{0 m}} r_{0 m}^{-\lambda} d r_{0 m} \\
& =\left\{\begin{array}{l}
C_{2}(\lambda)|h| \mid \ln \frac{R_{0 m}}{|h|} \text { при } \lambda=1, \\
\left.C_{2}(\lambda)|h|\left|R_{0 m}^{1-\lambda}-\right| h\right|^{1-\lambda} \mid
\end{array}\right.
\end{aligned}
$$

Учитьвая, что $\max R_{0 m} \leqslant D_{0}$ для фиксированной функции $\varphi(x)$, где $D_{0}-$ константа, зависящая от $\operatorname{supp} \varphi(x)$, из (4.11) получаем

$$
I_{3}(h) \leqslant \begin{cases}C_{3}(\lambda)|h| & \text { при } 0<\lambda<1 \\ C_{3}(\lambda)|h||\ln | h|| & \text { при } \lambda=1, \\ C_{3}(\lambda)|h|^{2-\lambda} & \text { при } 1<\lambda<2 .\end{cases}
$$

Из (4.9), (4.10) и (4.11) следует, что для $\left|\Delta u\left(M_{0 m}\right)\right|$ имеет место оценка (4.12).

Второй случай. Фиксируем некоторое $\delta>0$, тогда очевидно, что для всех точек $M_{0 m}$, находящихся на расстоянии $\rho_{0 m} \leqslant \delta$ от $\operatorname{supp} \varphi(x)$, имеет место оценка (4.12).

Третий случай. Рассмотрим теперь точки $M_{0 m}$, находящиеся на расстоянии $\rho_{0 m}>\delta$ от $\operatorname{supp} \varphi(x)$. 
В этом случае оценка квадратурной погрешности $\Delta u\left(M_{0 m}\right)$ будет следующей:

$$
\left|\Delta u\left(M_{0 m}\right)\right| \leqslant \sum_{k \in \varepsilon(\varphi)} \int_{D(k, h)} \frac{\left|\varphi(x)-\varphi\left(M_{0 k}\right)\right|}{r_{0 m}^{n+\lambda}} d x \leqslant C(\lambda) \frac{|h|}{\rho_{0 m}^{n+\lambda}} .
$$

Обозначим через $u(x, h)$ ступенчатую функцию, которую определим следуюшим образом: $u(x, h)=\left|\Delta u\left(M_{0 m}\right)\right|$ при $x \in D(m, h)$. Оценим величину $\Delta=$ $\int_{\mathbb{R}_{n}}|u(x, h)|^{2} d x$

Рассмотрим

$$
\Delta=\int_{J(h)}|u(x, h)|^{2} d x+\int_{\mathbb{R}_{n} \backslash J(h)}|u(x, h)|^{2} d x=\Delta_{1}+\Delta_{2},
$$

где $J(h)$ - область, состоящая из ячеек $D(m, h)$, центры которых (точки $\left.M_{0 m}\right)$ обладают свойством: либо $M_{0 m} \in \operatorname{supp} \varphi(x)$, либо расстояние от $M_{0 m}$ до $\operatorname{supp} \varphi(x)$ не превосходит $\delta$. Из определения области $J(h)$ и (4.12) следует, что

$$
\Delta_{1} \leqslant C(\lambda) O(|h|)
$$

где $O(|h|) \rightarrow 0$ при $|h| \rightarrow 0$.

При оценке слагаемого $\Delta_{2}$ можно без ограничения общности считать, что начало координат $O \in \operatorname{supp} \varphi(x)$. Пусть $r-$ текущий радиус-вектор точки $M \in$ $D(m, h) \subset \mathbb{R}_{n} \backslash \overline{J(h)}$, тогда ввиду условия $\rho_{0 m} \geqslant \delta$ будет выполняться $1 / \rho_{0 m} \leqslant$ $C / r$, где $C$ - константа, не зависящая от $D(m, h)$. Отсюда получим:

$$
\Delta_{2} \leqslant C h^{2} \int_{\delta}^{\infty} \frac{d r}{r^{n+2 \lambda+1}} \leqslant C_{1}|h|^{2}
$$

Из (4.14)-(4.16) следует неравенство

$$
\Delta \leqslant C_{1}(\lambda) O_{1}(|h|)
$$

где $O_{1}(h) \rightarrow 0$ при $|h| \rightarrow 0$.

ПРЕДЛОЖЕНИЕ 4.1. Если $\varphi(x) \in C_{0}^{\infty}\left(\mathbb{R}_{n}\right)$, то функиии $S(x, h), \pi S(x, h)=$ $S\left(M_{0 m}\right)$, сходятся в метрике $L_{2}\left(\mathbb{R}_{n}\right)$ при $|h| \rightarrow 0 \kappa u\left(x_{0}\right)=\int_{\mathbb{R}_{n}} \frac{\varphi(x) d x}{r^{n+\lambda}}$.

ДокАЗАтЕЛьство. Нужно заметить, что так как $\varphi(x) \in C_{0}^{\infty}\left(\mathbb{R}_{n}\right)$, то $u(x) \in$ $H_{r}\left(\mathbb{R}_{n}\right) \forall r$. Отсюда следует, что $u(x) \in C^{\infty}\left(\mathbb{R}_{n}\right), u(x) \in L_{2}\left(\mathbb{R}_{n}\right)$. Рассмотрим семейство функций $A(x, h)$ такое, что $\pi A(x, h)=u\left(M_{0 m}\right)$ (оператор $\pi$ задается в [2; определение 1.2]). Докажем равенство

$$
\lim _{|h| \rightarrow 0}\|u(x)-A(x, h)\|_{L_{2}\left(\mathbb{R}_{n}\right)}=0 .
$$


Возьмем произвольное $\varepsilon>0$. Пусть $K(R)$ - шар достаточно большого радиуса $h$, чтобы выполнялось неравенство

$$
\int_{\mathbb{R}_{n} \backslash K(R)}|u(x)|^{2} d x<\varepsilon .
$$

Рассмотрим множество $M_{h}=\bigcup_{m} D(m, h)$, где ячейки $D(m, h)$ имеют непустое пересечение с шаром $K(R)$. Тогда, так как $u(x) \in C^{\infty}\left(\mathbb{R}_{n}\right)$, найдется $\delta(\varepsilon)>0$ такое, что при $|h|<\delta(\varepsilon)$ будет выполняться $\mu\left(M_{h}\right)<2 \mu(K(R))$ и $|A(x, h)-u(x)|^{2}<\varepsilon /(2 \mu(K(R)))$ при $x \in D(m, h) \subset M_{h}, \mu\left(M_{h}\right)$ и $\mu(K(R))-$ объемы $M_{h}$ и $K(R)$. При $|h|<\delta(\varepsilon)$ имеет место неравенство

$$
\int_{M_{h}}|u(x)-A(x, h)|^{2} d x<\varepsilon .
$$

Без ограничения общности можно считать, что центр шара $K(R)$ находится в начале координат, а радиус $R$ выбран достаточно большим, так что граница $K(R)$ находится на положительном расстоянии $\delta_{0}$ от $\operatorname{supp} \varphi(x)$. Из этого замечания следует неравенство для точек $M_{0 m} \in \mathbb{R}_{n} \backslash K(R)$ :

$$
\left|A\left(M_{0 m}, h\right)\right|=\left|u\left(M_{0 m}\right)\right| \leqslant \int_{\mathbb{R}_{n}} \frac{|\varphi(x)| d x}{r_{0 m}^{n+\lambda}} \leqslant \frac{C}{R_{0 m}^{n+\lambda}},
$$

где $R_{0 m}$ - расстояние от начала координат до $M_{0 m}$.

Из (4.21) следует оценка

$$
\int_{\mathbb{R}_{n} \backslash K(R)}|A(x, h)|^{2} d x \leqslant C_{1} \int_{R}^{\infty} \frac{d r}{r^{n+2 \lambda+1}}=O(R),
$$

где $O(R) \rightarrow 0$ при $R \rightarrow \infty$.

Так как выбор параметров $h$ и $R$ происходит независимо, то из оценок (4.19), (4.20) и (4.22) следует (4.18).

Ввиду того что имеет место неравенство

$$
\|u(x)-S(x, h)\|_{L_{2}\left(\mathbb{R}_{n}\right)} \leqslant\|u(x)-A(x, h)\|_{L_{2}\left(\mathbb{R}_{n}\right)}+\|A(x, h)-S(x, h)\|_{L_{2}\left(\mathbb{R}_{n}\right)},
$$

из (4.17) и (4.18) следует предложение 4.1.

4.2. Квадратурные формулы для сингулярных интегралов. Рассмотрим сингулярньй интеграл

$$
u\left(x_{0}\right)=\int_{\mathbb{R}_{n}} \frac{f(\vec{\theta}) \varphi(x) d x}{r^{n}},
$$

где $r=\left|x^{0}-x\right|, f(\vec{\theta})$ - характеристика сингулярного интеграла, являюшаяся функцией вектора $\vec{\theta}=\frac{\mathbf{r}}{r}, f(\vec{\theta})$ удовлетворяет условию Липшица и, кроме того,

$$
\int_{S_{n}} f(\vec{\theta}) d S_{n}=0
$$


где $S_{n}-$ единичная сфера в $\mathbb{R}_{n}$.

Рассмотрим разбиение пространства $\mathbb{R}_{n}$ на ячейки $D(k, h)$ такое же, как и в п. 4.1. В дальнейшем будем считать, что $\varphi(x) \in C_{0}^{\infty}\left(\mathbb{R}_{n}\right)$. Для приближенного вычисления интеграла (4.23) в точках $M_{0 m}\left(m_{1} h_{1}, \ldots, m_{n} h_{n}\right)$ применим квадратурную формулу

$$
S\left(M_{0 m}\right)=\sum_{k} \varphi\left(M_{0 k}\right) \int_{D(k, h)} \frac{f(\vec{\theta}) d x}{r_{0 m}^{n}}
$$

где $r_{0 m}=\left|x^{0 m}-x\right|, x^{0 m}-$ координаты точки $M_{0 m}$. Оценим величину квадратурной погрешности $\Delta u\left(M_{0 m}\right)=u\left(M_{0 m}\right)-S\left(M_{0 m}\right)$. Имеет место равенство

$$
\begin{aligned}
\Delta u\left(M_{0 m}\right)= & \sum_{\substack{k \in \varepsilon(\varphi) \\
k \neq m}} \int_{D(k, h)} \frac{\left[\varphi(x)-\varphi\left(M_{0 k}\right)\right] f(\vec{\theta}) d x}{r_{0 m}^{n}} \\
& +\int_{D(m, h)} \frac{\left[\varphi(x)-\varphi\left(M_{0 m}\right)\right] f(\vec{\theta}) d x}{r_{0 m}^{n}}=I_{1}(h)+I_{2}(h),
\end{aligned}
$$

где $\varepsilon(\varphi)$ - множество номеров $k$ ячеек $D(k, h)$, имеющих непустое пересечение с $\operatorname{supp} \varphi(x)$.

Рассмотрим случай, когда $M_{0 m} \in \operatorname{supp} \varphi(x)$ либо расстояние от $M_{0 m}$ до $\operatorname{supp} \varphi(x)$ не превосходит фиксированного числа $\delta$. Будем считать, что $h=$ $\left(h_{1}, \ldots, h_{1}\right)$, т.е. шаг разбиения на каждой из осей выбран один и тот же; кроме того, $|h|=\sqrt{\sum_{i=1}^{n} h_{1}^{2}}=h_{1} \sqrt{n}<\delta_{0}=$ const. Возьмем шар $K_{R}\left(M_{0 m}\right)$ с центром в $M_{0 m}$ достаточно большого радиуса $R$ такой, чтобы ячейки $D(k, h)$, полностью вошедшие в $K_{R}\left(M_{0 m}\right)$, покрывали $\operatorname{supp} \varphi(x)$. Причем $R$ выбирается одним и тем же для всех $M_{0 m}$, такое число $R$ сушествует, так как $\varphi(x) \in C_{0}^{\infty}\left(\mathbb{R}_{n}\right)$. Далее имеем:

$$
\begin{aligned}
\left|I_{1}(h)\right| & \leqslant \sum_{\substack{k \in \varepsilon(\varphi) \\
k \neq m}} \int_{D(k, h)} \frac{\left|\varphi(x)-\varphi\left(M_{0 k}\right)\right||f(\vec{\theta})|}{r_{0 m}^{n}} d x \\
& \leqslant C_{1}|h| \int_{|h|}^{R} \frac{d r}{r} \leqslant C_{1}|h||\ln | h|| .
\end{aligned}
$$

Оценим слагаемое $I_{2}(h)$, используя определение сингулярного интеграла:

$$
\begin{aligned}
\left|I_{2}(h)\right| & \leqslant\left|\lim _{\varepsilon \rightarrow 0} \int_{D(m, h) \backslash D(\varepsilon)} \frac{f(\vec{\theta})\left[\varphi(x)-\varphi\left(M_{0 m}\right)\right]}{r^{n}} d x\right| \\
& \leqslant C \lim _{\varepsilon \rightarrow 0} \int_{\varepsilon}^{|h| \sqrt{n}} d r \leqslant C_{1}|h|,
\end{aligned}
$$

где $D(\varepsilon)$ - шар радиуса $\varepsilon$ с центром в $M_{0 m}$.

Из оценок (4.26) и (4.27) следует неравенство

$$
\left|\Delta u\left(M_{0 m}\right)\right| \leqslant C|h||\ln | h|| .
$$


Рассмотрим теперь случай, когда точки $M_{0 m}$ находятся на расстоянии $\rho_{0 m}>\delta$ от $\operatorname{supp} \varphi(x)$. Для этих точек получим оценку

$$
\left|\Delta u\left(M_{0 m}\right)\right| \leqslant \sum_{k \in \varepsilon(\varphi)} \int_{D(k, h)} \frac{|f(\vec{\theta})|\left|\varphi(x)-\varphi\left(M_{0 k}\right)\right| d x}{r_{0 m}^{n}} \leqslant \frac{C|h|}{\rho_{0 m}^{n}} .
$$

Обозначим через $u(x, h)$ ступенчатую функцию, которую определим следующим образом: $u(x, h)=\left|\Delta u\left(M_{0 m}\right)\right|$ при $x \in D(m, h)$. Аналогично (4.17) получается соотношение

$$
\Delta=\int_{\mathbb{R}_{n}}|u(x, h)|^{2} d x=O(|h|),
$$

где $O(|h|) \rightarrow 0$ при $|h| \rightarrow 0$.

ПРЕДЛОЖЕНИЕ 4.2. Если $\varphi(x) \in C_{0}^{\infty}\left(\mathbb{R}_{n}\right)$, то функиии $S(x, h), \pi S(x, h)=$ $S\left(M_{0 m}\right)$, сходятся в метрике $L_{2}\left(\mathbb{R}_{n}\right)$ при $|h| \rightarrow 0 \kappa u\left(x_{0}\right)=\int_{\mathbb{R}_{n}} \frac{f(\vec{\theta}) \varphi(x) d x}{r^{n}}$.

Доказательство аналогично доказательству предложения 4.1.

\section{3. Квадратурные формулы для суперсингулярных интегралов вида}

$$
u\left(x_{0}\right)=\int_{\mathbb{R}_{n}} \frac{\varphi(x) d x}{r^{n+2 p+\lambda}},
$$

где $0<\lambda<2, p>0, p \in \mathbb{N}$.

Для преобразования Фурье обобшенных функций $f_{1}(x)=r^{-n-2 p-\lambda}$ и $f_{2}(x)=$ $r^{-n-\lambda}$ имеет место (см. [4])

$$
\begin{aligned}
F\left(r^{-n-2 p-\lambda}\right) & =\frac{\Gamma\left(-p-\frac{\lambda}{2}\right)}{\Gamma\left(\frac{n+2 p+\lambda}{2}\right)} 2^{-2 p-\lambda} \pi^{n / 2}|\sigma|^{2 p+\lambda} \\
F\left(r^{-n-\lambda}\right) & =\frac{\Gamma\left(-\frac{\lambda}{2}\right)}{\Gamma\left(\frac{n+\lambda}{2}\right)} 2^{-\lambda} \pi^{n / 2}|\sigma|^{\lambda}
\end{aligned}
$$

где $\Gamma(t)$ - гамма-функция.

Исходя из последних соотношений, символ (4.30) будем понимать следуюшим образом:

$$
u\left(x_{0}\right)=C(\lambda, p) \int_{\mathbb{R}_{n}} \frac{\Delta^{p} \varphi(x) d x}{r^{n+\lambda}}
$$

где константа $C(\lambda, p)$ определяется из (4.31), $\Delta$ - оператор Лапласа, интеграл (4.32) понимается по Адамару.

Далее будем использовать такие же обозначения, как и в пп. 4.1 и 4.2.

$\mathrm{B}$ дальнейшем будем считать, что $\varphi(x) \in C_{0}^{\infty}\left(\mathbb{R}_{n}\right)$. Для приближенного вычисления интеграла (4.32) в точках $M_{0 m}$ используем квадратурную формулу

$$
S\left(M_{0 m}\right)=C(\lambda, p) \sum_{k \in \varepsilon(\varphi)} \int_{D(k, h)} \frac{\Delta_{h}^{p} \varphi\left(M_{0 k}\right) d x}{r_{0 m}^{n+\lambda}}
$$


где $\Delta_{h}^{p}$ - итерированный разностный оператор Лапласа порядка $p, \varepsilon(\varphi)$ - множество номеров $k$ ячеек $D(k, h)$, имеющих непустое пересечение с $\operatorname{supp} \Delta_{h}^{p} \varphi\left(M_{0 k}\right)$.

Оценим величину квадратурной погрешности $\Delta u\left(M_{0 m}\right)=u\left(M_{0 m}\right)-S\left(M_{0 m}\right)$. Преобразуем $\Delta u\left(M_{0 m}\right)$ :

$$
\begin{aligned}
\Delta u\left(M_{0 m}\right)= & C(\lambda, p) \sum_{k \in \varepsilon(\varphi)} \int_{D(k, h)} \frac{\Delta^{p} \varphi(x)-\Delta^{p} \varphi\left(M_{0 k}\right)}{r_{0 m}^{n+\lambda}} d x \\
& +C(\lambda, p) \sum_{k \in \varepsilon(\varphi)} \int_{D(k, h)} \frac{\Delta^{p} \varphi\left(M_{0 k}\right)-\Delta_{h}^{p} \varphi\left(M_{0 k}\right)}{r_{0 m}^{n+\lambda}} d x \\
= & \Delta_{1} u\left(M_{0 m}\right)+\Delta_{2}\left(M_{0 m}\right) .
\end{aligned}
$$

Для погрешности $\Delta_{1} u\left(M_{0 m}\right)$ справедливы все результаты п. 4.1. Перейдем к оценке величины $\Delta_{2} u\left(M_{0 m}\right)$, для этого выполним преобразования:

$$
\begin{aligned}
\left|\Delta_{2} u\left(M_{0 m}\right)\right| \leqslant & |C(\lambda, p)|\left|\Delta^{p} \varphi\left(M_{0 m}\right)-\Delta_{h}^{p} \varphi\left(M_{0 m}\right)\right| \cdot\left|\int_{D(m, h)} \frac{d x}{r_{0 m}^{n+\lambda}}\right| \\
& +\sum_{\substack{k \in \in(\varphi) \\
k \neq m}}|C(\lambda, p)|\left|\Delta^{p} \varphi\left(M_{0 k}\right)-\Delta_{h}^{p} \varphi\left(M_{0 k}\right)\right| \cdot \int_{D(k, h)} \frac{d x}{r_{0 m}^{n+\lambda}} \\
= & B_{1}(h)+B_{2}(h) .
\end{aligned}
$$

Так как $\varphi(x) \in C_{0}^{\infty}\left(\mathbb{R}_{n}\right)$, то из [2; предложение 5.8] следует равномерная оценка $\left|\Delta^{p} \varphi\left(M_{0 k}\right)-\Delta_{h}^{p} \varphi\left(M_{0 k}\right)\right| \leqslant k \Omega_{h}^{2 / n}$, где $k$ не зависит от $h, \Omega_{h}=\prod_{j=1}^{n} h_{j}$.

Из [2; условие (2.3)] получим неравенство

$$
\left|\Delta^{p} \varphi\left(M_{0 k}\right)-\Delta_{h}^{p} \varphi\left(M_{0 k}\right)\right| \leqslant k_{1}|h|^{2} .
$$

Пусть $|h|<\delta_{0}$; рассмотрим случай, когда для точек $M_{0 m}$ выполняется условие: либо $M_{0 m} \in \operatorname{supp} \Delta_{h}^{p} \varphi\left(M_{0 k}\right)$, либо расстояние от $M_{0 m}$ до $\operatorname{supp} \Delta_{h}^{p} \varphi\left(M_{0 k}\right)$ не превосходит некоторого числа $\delta>0$.

Из определения интегралов по Адамару [2; формула (3.9)] следуют оценки

$$
\sum_{k \neq m} \int_{D(k, h)} \frac{d x}{r_{0 m}^{n+\lambda}} \leqslant \frac{C}{|h|^{\lambda}} ; \quad\left|\int_{D(m, h)} \frac{d x}{r_{0 m}^{n+\lambda}}\right| \leqslant \frac{C}{|h|^{\lambda}} .
$$

Из оценок (4.35)-(4.37) вытекает неравенство

$$
\left|\Delta_{2} u\left(M_{0 m}\right)\right| \leqslant C_{1}|h|^{2-\lambda} .
$$

Рассмотрим случай, когда точки $M_{0 m}$ находятся на расстоянии $\rho_{0 m} \geqslant \delta$ от $\operatorname{supp} \Delta_{h}^{p} \varphi\left(M_{0 k}\right)$. Для этих точек имеет место неравенство

$$
\begin{aligned}
\left|\Delta_{2} u\left(M_{0 m}\right)\right| & \leqslant|C(\lambda, p)| \sum_{k \in \varepsilon(\varphi)} \int_{D(k, h)} \frac{\left|\Delta^{p} \varphi\left(M_{0 k}\right)-\Delta_{h}^{p} \varphi\left(M_{0 k}\right)\right|}{r_{0 m}^{n+\lambda}} d x \\
& \leqslant \frac{C_{1}(\lambda, p)|h|^{2}}{\rho_{0 m}^{n+\lambda}} .
\end{aligned}
$$

Так как для $\Delta_{1} u\left(M_{0 m}\right)$ справедливы все результаты п. 4.1, то из (4.39) для функции $u(x, h), \pi u(x, h)=\Delta u\left(M_{0 m}\right)$, выполняется соотношение

$$
\int_{\mathbb{R}_{n}}|u(x, h)|^{2} d x=O(|h|),
$$

где $O(|h|) \rightarrow 0$ при $|h| \rightarrow 0$. 
ПРЕДЛОЖЕНИЕ 4.3. Если $\varphi(x) \in C_{0}^{\infty}\left(\mathbb{R}_{n}\right)$, то функиии $S(x, h), \pi S(x, h)=$ $S\left(M_{0 m}\right)$, сходятся в метрике $L_{2}\left(\mathbb{R}_{n}\right)$ при $|h| \rightarrow 0 \kappa$

$$
u\left(x_{0}\right)=C(\lambda, p) \int_{\mathbb{R}_{n}} \frac{\Delta^{p} \varphi(x) d x}{r^{n+\lambda}} .
$$

Доказательство аналогично доказательству предложения 4.1.

\section{§5. Устойчивость дискретных операторов в пространствах дробных отношений}

В дальнейшем будем предполагать, что $\Omega$ - открытая ограниченная область с липшицевой границей, нормальная, допускающая разбиение (см. [2; определения $6.1-6.4,6.6,6.9])$.

5.1. Дискретные вихревые операторы. Дискретные вихревые операторы задаются соотношениями (3.43) и (3.44). Здесь удобно представить их в следующем, эквивалентном виде:

$$
\begin{gathered}
\pi \mathrm{T}_{h, \lambda} u(x, h)=\sum_{k} u(k) \gamma(k, m), \\
\gamma(k, m)=\int_{D(k, h)} \frac{d x}{r_{0 m}^{n+\lambda}}, \quad 0<\lambda<2,
\end{gathered}
$$

где $r_{0 m}=\left|M M_{0 m}\right|$ (см. п. 4.1), оператор $\pi$ задается в [2; определение 1.2], $u(x, h) \in$ $\stackrel{\circ}{M}\left(r, h, \Omega_{1 h}\right)$ (см. [2; определение 6.2]), суммирование в (5.1) происходит по всем номерам $k$ ячеек $D(k, h)$ таких, что $D(k, h) \subset \Omega_{1 h}$.

Из предложения 3.4 следует, что семейство операторов $\mathrm{T}_{h, \lambda}$ ограниченно отображает пространства $M(r, h)$ в $M(r-\lambda, h)$. Из определения пространств $\stackrel{\circ}{M}\left(r, h, \Omega_{1 h}\right)$ и $M\left(r, h, \Omega_{1 h}\right)$ вытекает, что семейство операторов $\mathrm{P}_{h} \mathrm{~T}_{h, \lambda}$ ограниченно отображает $\stackrel{\circ}{M}\left(\lambda / 2, h, \Omega_{1 h}\right)$ в $M\left(-\lambda / 2, h, \Omega_{1 h}\right)$, где $\mathrm{P}_{h}$ - оператор сужения на $\Omega_{1 h}$.

Исследуем на устойчивость семейство операторов $\mathrm{P}_{h} \mathrm{~T}_{h, \lambda}$ при отображении пространств $\stackrel{\circ}{M}\left(\lambda / 2, h, \Omega_{1 h}\right)$ в $M\left(-\lambda / 2, h, \Omega_{1 h}\right)$.

Легко проверить равенство $\gamma(k, m)=\gamma(m, k)$, которое выполняется для любых $k$ и $m$. Рассмотрим линейный функционал $I=(2 \pi)^{-n} l(\nu)$ над $M\left(-\lambda / 2, h, \Omega_{1 h}\right)$, определяемый элементом $u(x, h) \in \stackrel{\circ}{M}\left(\lambda / 2, h, \Omega_{1 h}\right)$. Тогда на основании $[2 ;(6.5)]$ получим

$$
\begin{aligned}
I & =\frac{1}{(2 \pi)^{n}} l\left(\mathrm{P}_{h} \mathrm{~T}_{h} u(x, h)\right)=\sum_{m \in \Lambda} \Omega_{h} \overline{u(m)} \sum_{k \in \Lambda} u(k) \gamma(k, m) \\
& =\Omega_{h} \sum_{m \in \Lambda} \overline{u(m)}\left[\sum_{\substack{k \in \Lambda \\
k \neq m}} u(k) \gamma(k, m)+\gamma(m, m) \cdot u(m)\right]
\end{aligned}
$$

где $\Lambda$ - множество номеров $k$ таких, что $D(k, h) \subset \Omega_{1 h} ; \pi u(x, h)=u(k)$; $\Omega_{h}=\prod_{j=1}^{n} h_{j}$. Из определения интеграла по Адамару [2; формула (3.9)] следует: $\gamma(k, m)>0 \forall k, m$ при $k \neq m ; \sum_{k} \gamma(k, m)=0, \gamma(m)=\sum_{k \in \Lambda} \gamma(k, m)<0$. 
Используя сделанные замечания, преобразуем (5.2):

$$
\begin{aligned}
I & =\Omega_{h} \sum_{m \in \Lambda} \overline{u(m)}\left[\sum_{\substack{k \in \Lambda \\
k \neq m}} u(k) \gamma(k, m)-\sum_{\substack{k \in \Lambda \\
k \neq m}} \gamma(k, m) u(m)+\gamma(m) u(m)\right] \\
& =\Omega_{h} \sum_{m \in \Lambda} \overline{u(m)} \sum_{k \in \Lambda}[u(k)-u(m)]+\Omega_{h} \sum_{m \in \Lambda} \gamma(m)|u(m)|^{2} .
\end{aligned}
$$

Заменяя переменные суммирования $k$ на $m, m$ на $k$, а также учитывая, что $\gamma(k, m)=\gamma(m, k)$, из соотношения (5.3) получим:

$$
I=\Omega_{h} \sum_{k \in \Lambda} \overline{u(k)} \sum_{m \in \Lambda} \gamma(k, m)[u(m)-u(k)]+\Omega_{h} \sum_{m \in \Lambda} \gamma(m)|u(m)|^{2} .
$$

Из (5.3) и (5.4) следует, что

$$
I=-\frac{\Omega_{h}}{2} \sum_{m \in \Lambda} \sum_{k \in \Lambda} \gamma(k, m)|u(k)-u(m)|^{2}+\Omega_{h} \sum_{m \in \Lambda} \gamma(m)|u(m)|^{2} .
$$

Докажем, что $|\gamma(m)|>C>0$. Действительно, по условию область $\Omega$ является ограниченной, поэтому существует такое число $R_{0}>0$, что для любой точки $M_{0 m} \in \Omega$ шар $K\left(R_{0}, M_{0 m}\right)$ с центром в $M_{0 m}$ и радиусом $R_{0}$ содержит $\Omega$. Отсюда имеем:

$$
|\gamma(m)|=\left|\sum_{k \in \Lambda} \gamma(k, m)\right| \geqslant\left|\int_{K\left(R_{0}, M_{0 m}\right)} \frac{d x}{r_{0 m}^{n+\lambda}}\right| \geqslant C .
$$

Из (5.5) и (5.6) вытекает неравенство

$$
|I| \geqslant C \Omega_{h} \sum_{m \in \Lambda}|u(m)|^{2}
$$

С другой стороны, учитывая, что $u(k)=0, u(m)=0$ при $k, m \notin \Lambda$, получаем:

$$
I=\Omega_{h} \sum_{m} \overline{u(m)} \sum_{k} u(k) \gamma(k, m)=\Omega_{h} \sum_{m} \overline{u(m)} \sum_{k} \gamma(k, m)[u(k)-u(m)] .
$$

Переименуем переменные суммирования в (5.8), тогда

$$
I=-\Omega_{h} \sum_{k} \overline{u(k)} \sum_{m} \gamma(k, m)[u(k)-u(m)] .
$$

Из (5.8) и (5.9) следует соотношение

$$
I=-\frac{\Omega_{h}}{2} \sum_{k} \sum_{m} \gamma(k, m)|u(k)-u(m)|^{2} .
$$

Далее учтем, что для $\gamma(k, m)$ при $k \neq m$ выполняются неравенства

$$
C_{1} \frac{\Omega_{h}}{(|k-m||h|)^{n+\lambda}} \leqslant \gamma(k, m) \leqslant C_{2} \frac{\Omega_{h}}{(|k-m||h|)^{n+\lambda}} .
$$


Из (5.10) и (5.11) получим оценку

$$
|I| \geqslant C_{3} \Omega_{h} \sum_{k \neq m} \frac{\Omega_{h}}{(|k-m||h|)^{n+\lambda}}|u(k)-u(m)|^{2} .
$$

Из (5.7) и (5.12), а также из [2; предложение 4.1] следует оценка

$$
|I| \geqslant C_{4}\|u(x, h)\|_{M\left(\lambda / 2, h, \Omega_{1 h}\right)}^{2},
$$

где $C_{4}$ не зависит от $h$.

Используя (5.13), получаем

$$
\begin{aligned}
C_{4}\|u(x, h)\|_{\stackrel{\circ}{\circ}\left(\lambda / 2, h, \Omega_{1 h}\right)}^{2} \leqslant|I| \leqslant & \frac{1}{(2 \pi)^{n}}\|u(x, h)\|_{\stackrel{\circ}{M}\left(\lambda / 2, h, \Omega_{1 h}\right)} \\
& \times\left\|\mathrm{P}_{h} \mathrm{~T}_{h, \lambda} u(x, h)\right\|_{M\left(-\lambda / 2, h, \Omega_{1 h}\right)} .
\end{aligned}
$$

Из неравенства (5.14) и определения 2.1 следует

ПРЕДЛОЖЕНИЕ 5.1. Оحраниченное семейство вихревых дискретных операторов $\mathrm{P}_{h} \mathrm{~T}_{h, \lambda}$ устойчиво отображсает $\stackrel{\circ}{M}\left(\lambda / 2, h, \Omega_{1 h}\right)$ в $M\left(-\lambda / 2, h, \Omega_{1 h}\right)$.

5.2. Итерированный разностный оператор Лапласа порядка $m$. Предварительно докажем

ПРЕДЛОЖЕНИЕ 5.2. Для любой $u(x, h) \in \stackrel{\circ}{M}\left(\lambda, h, \Omega_{1 h}\right), r \geqslant 0$, выполняется неравенство

$$
\Omega_{h} \int_{-\pi}^{\pi} \cdots \int_{-\pi}^{\pi}\left(\sum_{j=1}^{n}\left(\frac{t_{j}}{h_{j}}\right)^{2}\right)^{r}\left|\widehat{u}_{c}(t)\right|^{2} d t \geqslant C\|u(x, h)\|_{M\left(r, h, \Omega_{1 h}\right)}^{2},
$$

$\widehat{u}_{c}(t)$ - дискретное преобразование Фурье сеточной функции $\pi u(x, h)=u(k)$, $C>0$ не зависит от $h=\left(h_{1}, \ldots, h_{n}\right)$.

ДокАЗАТЕЛЬСтво. Ввиду формулы (2.2) из [2] достаточно доказать неравенство

$$
\Omega_{h} \int_{-\pi}^{\pi} \ldots \int_{-\pi}^{\pi}\left(\sum_{j=1}^{n}\left(\frac{t_{j}}{h_{j}}\right)^{2}\right)^{r}\left|\widehat{u}_{c}(t)\right|^{2} d t \geqslant C_{1} \Omega_{h} \int_{U}\left|\widehat{u}_{c}(t)\right|^{2} d t,
$$

где $C_{1}$ не зависит от $h$.

Далее имеем

$$
\begin{aligned}
\left|\widehat{u}_{c}(t)\right|=\left|\sum_{k} u(k) e^{i(k, t)}\right| & \leqslant \frac{1}{\Omega_{h}} \cdot \sqrt{\sum_{k}|u(k)|^{2} \Omega_{h}} \cdot \sqrt{\sum_{k} 1^{2} \Omega_{h}} \\
& \leqslant \frac{\sqrt{V(\Omega)}}{\Omega_{h}}\|u(x, h)\|_{L_{2}\left(\Omega_{1 h}\right)}
\end{aligned}
$$


где суммирование происходит по всем $k$ таким, что $D(k, h) \subset \Omega_{1 h}, V(\Omega)$ - объем области $\Omega, \Omega_{h}=\prod_{j=1}^{n} h_{j}$.

Так как $\Omega$ - ограниченная область, то найдется $\delta>0$ такое, что значения параметра $h=\left(h_{1}, \ldots, h_{n}\right)$, для которых имеет смысл пространство $\stackrel{\circ}{M}\left(r, h, \Omega_{1 h}\right)$, удовлетворяют условию $|h|<\delta$. Поэтому существует такое число $A(\Omega)>2$, что множество $U_{A} \subset U=\prod_{j=1}^{n}\left[-\pi \leqslant t_{j} \leqslant \pi\right]$, где

$$
U(A)=\prod_{j=1}^{n} I_{j}(A), \quad I_{j}(A)=\left\{t_{j}:\left|t_{j}\right| \leqslant \frac{h_{j}}{A \sqrt[n]{V(\Omega)}}\right\}
$$

Из (5.17) получим

$$
\Omega_{h} \int_{U(A)}\left|\widehat{u}_{c}(t)\right|^{2} d t \leqslant \Omega_{h} \frac{V(\Omega)}{\Omega_{h}^{2}}\|u(x, h)\|_{L_{2}\left(\Omega_{1 h}\right)}^{2} \cdot \frac{\Omega_{h}}{V(\Omega)(A)^{n}} .
$$

Из конструкции множества $U(A)$ и неравенства (5.18) имеем

$\Omega_{h} \int_{U}\left(\sum_{j=1}^{n}\left(\frac{t_{j}}{h_{j}}\right)^{2}\right)^{r}\left|\widehat{u}_{c}(t)\right|^{2} d t \geqslant C_{1} \Omega_{h} \int_{U \backslash U(A)}\left|\widehat{u}_{c}(t)\right|^{2} d t \geqslant C_{2} \Omega_{h} \int_{U}\left|\widehat{u}_{c}(t)\right|^{2} d t$,

где $C_{2}>0$ не зависит от $h$. Из последнего неравенства следует предложение 5.2.

ПРЕДЛОЖенИЕ 5.3. Семейство операторов $\mathrm{P}_{h} \mathrm{~T}_{h}=\mathrm{P}_{h} \Delta_{h p}^{m}$, где $\Delta_{h p}^{m}-$ итерированный разностный оператор Лапласа порядка $m$, устойчиво отображает пространства $\stackrel{\circ}{M}\left(m, h, \Omega_{1 h}\right)$ в пространства $M\left(-m, h, \Omega_{1 h}\right)$.

ДокАЗАТЕЛьство. Из п. 3.4.б) следует, что семейство операторов $\mathrm{P}_{h} \mathrm{~T}_{h}$ ограниченно отображает пространства $\stackrel{\circ}{M}\left(m, h, \Omega_{1 h}\right)$ в $M\left(-m, h, \Omega_{1 h}\right)$.

Рассмотрим линейный функционал $I=(2 \pi)^{-n} l(V)$ над $M\left(-m, h, \Omega_{1 h}\right)$, определяемый элементом $u(x, h) \in \stackrel{\circ}{M}\left(m, h, \Omega_{1 h}\right)$. Тогда на основании [2; формула (6.5)] получим:

$$
\begin{aligned}
|I| & =\left|\frac{1}{(2 \pi)^{n}} l\left(\mathrm{P}_{h} \mathrm{~T}_{h} u(x, h)\right)\right|=\left.\left|\frac{\Omega_{h}}{(2 \pi)^{n}} \int_{U} \widehat{A}_{c}(t)\right| \widehat{u}_{c}(t)\right|^{2} d t \mid \\
& =\left.\left|\frac{\Omega_{h}}{(2 \pi)^{n}} \int_{U}(-1)^{m} 4^{m}\left[\sum_{k=1}^{n} \frac{1}{h_{k}^{2}} \sin ^{2} \frac{t_{k}}{2}\right]^{m}\right| \widehat{u}_{c}(t)\right|^{2} d t \mid \\
& \geqslant C_{1} \Omega_{h} \int_{U}\left(\sqrt{\sum_{k=1}^{n}\left(\frac{t_{k}}{h_{k}}\right)^{2}}\right)^{2 m}\left|\widehat{u}_{c}(t)\right|^{2} d t \geqslant C_{2}\|u(x, h)\|_{\substack{\circ \\
M\left(m, h, \Omega_{1 h}\right)}}^{2}
\end{aligned}
$$

где

$$
\widehat{A}_{c}(t)=(-1)^{m} 4^{m}\left[\sum_{k=1}^{n} \frac{1}{h_{k}^{2}} \sin ^{2} \frac{t_{k}}{2}\right]^{m}
$$

- символ оператора $\Delta_{h p}^{m} ; C_{1}$ и $C_{2}$ не зависят от $h ;$ при получении неравенства (5.19) было использовано предложение 5.2. Из неравенства (5.19) следует устойчивость семейства операторов $\mathrm{P}_{h} \mathrm{~T}_{h}$. Предложение 5.3 доказано. 
5.3. Гиперсингулярные операторы. Гиперсингулярные дискретные операторы $\mathrm{T}_{h}(m, \lambda)$ определяются равенством $(3.50)$.

ПРЕДЛОЖенИЕ 5.4. Семейство операторов $\mathrm{P}_{h} \mathrm{~T}_{h}(m, \lambda)$ устойчиво отображает пространства $\stackrel{\circ}{M}\left(m+\lambda / 2, h, \Omega_{1 h}\right)$ в $M\left(-m-\lambda / 2, h, \Omega_{1 h}\right)$.

ДокАЗАТЕЛЬСТво. Из п. 3.4 следует, что семейство $\mathrm{P}_{h} \mathrm{~T}_{h}(m, \lambda)$ ограниченно отображает пространства $\stackrel{\circ}{M}\left(m+\lambda / 2, h, \Omega_{1 h}\right)$ в $M\left(-m-\lambda / 2, h, \Omega_{1 h}\right)$. Рассмотрим линейный функционал $I=(2 \pi)^{-n} l(V)$ над $M\left(-m-\lambda / 2, h, \Omega_{1 h}\right)$, определяемый элементом $u(x, h) \in \stackrel{\circ}{M}\left(m+\lambda / 2, h, \Omega_{1 h}\right)$. Тогда на основании (6.5) из [2] получим

$$
\begin{aligned}
|I| & =\left|\frac{1}{(2 \pi)^{n}} l\left(\mathrm{P}_{h} \mathrm{~T}_{h}(m, \lambda) u(x, h)\right)\right|=\left.\left|\frac{\Omega_{h}}{(2 \pi)^{n}} \int_{U} \widehat{A}_{c}(t)\right| \widehat{u}_{c}(t)\right|^{2} d t \mid \\
& =\left.\left|\frac{\Omega_{h}}{(2 \pi)^{n}} \int_{U}(-1)^{m} 4^{m}\left[\sum_{k=1}^{n} \frac{1}{h_{k}^{2}} \sin ^{2} \frac{t_{k}}{2}\right]^{m} \cdot J(t, \lambda) \cdot\right| \widehat{u}_{c}(t)\right|^{2} d t \mid \\
& \geqslant C_{1} \Omega_{h} \int_{U}\left(\sqrt{\sum_{k=1}^{n}\left(\frac{t_{k}}{h_{k}}\right)^{2}}\right)^{2 m} \cdot \frac{|t|^{\lambda}}{h_{1}^{\lambda}}\left|\widehat{u}_{c}(t)\right|^{2} d t \\
& \geqslant C_{2} \Omega_{h} \int_{U}\left(\sum_{k=1}^{n}\left(\frac{t_{k}}{h_{k}}\right)^{2}\right)^{m+\lambda / 2}\left|\widehat{u}_{c}(t)\right|^{2} d t \\
& \geqslant C_{3}\|u(x, h)\|_{M\left(m+\lambda / 2, h, \Omega_{1 h}\right)},
\end{aligned}
$$

где

$$
\widehat{A}_{c}(t)=(-1)^{m} 4^{m}\left[\sum_{k=1}^{n} \frac{1}{h_{k}^{2}} \sin ^{2} \frac{t_{k}}{2}\right]^{2} \cdot J(t, \lambda)
$$

- символ гиперсингулярного дискретного оператора, $J(t, \lambda)$ - символ вихревого дискретного оператора; для $J(t, \lambda)$ имеет место неравенство $|J(t, \lambda)| \geqslant C_{1}|t|^{\lambda} / h_{1}^{\lambda}$ (см. $[2 ;(3.15)])$.

Из неравенства (5.20) следует устойчивость семейства $\mathrm{T}_{h}(m, \lambda)$. Предложение 5.4 доказано.

5.4. Разностный эллиптический оператор 2-го порядка с переменными коэффициентами. Рассмотрим оператор

$$
\mathrm{L}(u)=\sum_{i=1}^{n} \frac{\partial}{\partial x_{i}} \sum_{j=1}^{n} a_{i j}(x) \frac{\partial u}{\partial x_{j}},
$$

удовлетворяющий условию равномерной эллиптичности в ограниченной области $\Omega$ евклидова пространства $\mathbb{R}_{n}$ :

$$
\nu \sum_{i=1}^{n} \xi_{i}^{2} \leqslant \sum_{i=1}^{n} \sum_{j=1}^{n} a_{i j}(x) \xi_{i} \xi_{j} \leqslant \mu \sum_{i=1}^{n} \xi_{i}^{2},
$$

где $\nu, \mu>0$ - некоторые константы, $a_{i j}(x)=a_{j i}(x), a_{i j}(x) \in C^{1}(\bar{\Omega})$. 
Определим дискретный оператор $\mathrm{L}_{h} u(x, h)$ по формуле

$$
\mathrm{L}_{h} u(x, h)=\sum_{i=1}^{n} \mathrm{~T}_{\text {лев }}\left(x_{i}\right) \sum_{j=1}^{n} a_{i j}(x, h) \mathrm{T}_{\text {пр }}\left(x_{j}\right) u(x, h)
$$

где $u(x, h) \in \stackrel{\circ}{M}\left(r, h, \Omega_{1 h}\right), \mathrm{T}_{\text {пр }}\left(x_{i}\right), \mathrm{T}_{\text {лев }}\left(x_{i}\right)$ - правое и левое разностные отношения по переменной $x_{i}$ (см. [2; пример 1.2]); $a_{i j}(x, h)=a_{i j}\left(k_{1} h_{1}, \ldots, k_{n} h_{n}\right)$ при $x \in D\left(k_{1}, \ldots, k_{n}, h_{1}, \ldots, h_{n}\right) \subset \Omega_{1 h}$.

ПРЕДЛОЖЕНИЕ 5.5. Семейство операторов $\mathrm{P}_{h} \mathrm{~L}_{h}$ устойчиво отображсает пространства $\stackrel{\circ}{M}\left(1, h, \Omega_{1 h}\right)$ в $M\left(-1, h, \Omega_{1 h}\right)$.

ДокАЗАтЕльство. Из условия $a_{i j}(x) \in C^{1}\left(\bar{\Omega}_{1 h}\right)$ и п. 3.4.а) следует, что семейство операторов $\mathrm{P}_{h} \mathrm{~L}_{h}$ ограниченно отображает пространства $\stackrel{\circ}{M}\left(1, h, \Omega_{1 h}\right)$ в $M\left(-1, h, \Omega_{1 h}\right)$. Введем обозначение: $\nu_{i}(x, h)=\sum_{j=1}^{n} a_{i j}(x, h) \mathrm{T}_{\text {пр }}\left(x_{j}\right) u(x, h)$, $\widehat{\nu}_{i}(\varphi)$ - дискретное преобразование Фурье сеточной функции $\nu_{i}(k)=\pi \nu_{i}(x, h)($ см. [2; определение 1.2]).

Рассмотрим линейный функционал $I=(2 \pi)^{-n} l(V(x))$ над $M\left(-1, h, \Omega_{1 h}\right)$, определяемый элементом $u(x, h) \in \stackrel{\circ}{M}\left(1, h, \Omega_{1 h}\right)$. Тогда на основании [2; формула (6.5)] получим

$$
\begin{aligned}
I & =\frac{1}{(2 \pi)^{n}} l\left(\mathrm{P}_{h} \mathrm{~L}_{h} u(x, h)\right) \\
& =\frac{\Omega_{h}}{(2 \pi)^{n}} \sum_{k=1}^{n} \int_{U} \widehat{\nu}_{k}(\varphi) \frac{1-e^{i \varphi_{k}}}{h_{k}} \overline{\widehat{u}_{c}(\varphi)} d \varphi \\
& =-\frac{\Omega_{h}}{(2 \pi)^{n}} \sum_{k=1}^{n} \int_{U} \widehat{\nu}_{k}(\varphi) \frac{\overline{-1+e^{-i \varphi_{k}}}}{h_{k}} \cdot \overline{\widehat{u}_{c}(\varphi)} d \varphi \\
& =-\int_{\mathbb{R}_{n}} \sum_{k=1}^{n} \sum_{j=1}^{n} a_{k j}(x, h)\left(\mathrm{T}_{\text {пр }}\left(x_{k}\right) u(x, h)\right)^{2} d x .
\end{aligned}
$$

Из (5.23) и (5.24) получим неравенство

$$
|I| \geqslant \nu \int_{\mathbb{R}_{n}} \sum_{k=1}^{n}\left[\mathrm{~T}_{\text {пр }}\left(x_{k}\right) u(x, h)\right]^{2} d x \geqslant C_{0} \Omega_{h} \int_{U}\left(\sqrt{\sum_{k=1}^{n}\left(\frac{t_{k}}{h_{k}}\right)^{2}}\right)^{2}\left|\widehat{u}_{c}(t)\right| d t .
$$

Из (5.25) и предложения 5.2 вытекает оценка

$$
\left\|\mathrm{P}_{h} \mathrm{~L}_{h} u(x, h)\right\|_{M\left(-1, h, \Omega_{1 h}\right)} \geqslant C_{1}\|u(x, h)\|_{\stackrel{\circ}{\circ\left(1, h, \Omega_{1 h}\right)}},
$$

где $C_{1}$ не зависит от $h$.

Из последнего неравенства следует предложение 5.5 . 


\section{§6. Исследование некоторых уравнений}

ОПРЕДЕЛЕНИЕ 6.1. Семейство ступенчатых функций $u(x, h)$ будем называть липшицевым, если выполняются условия:

1. $|u(x, h)| \leqslant C_{1}$, где $C_{1}$ не зависит от $h$;

2. для любых $x_{1} \in D(k, h)$ и $x_{2} \in D(m, h)$

$$
\left|u\left(x_{1}, h\right)-u\left(x_{2}, h\right)\right| \leqslant C_{2}|m-k| \cdot|h|,
$$

где $C_{2}$ не зависит от $h$.

ПРЕДЛОЖЕНИЕ 6.1. Если $u(x, h) \in M(r, h),|r|<1, a u_{0}(x, h)-$ липшицево семейство функиий, то имеет место неравенство

$$
\|\nu(x, h)\|_{M(r, h)} \leqslant C(r)\|u(x, h)\|_{M(r, h)},
$$

где $C$ не зависит от $h, \nu(x, h)=u(x, h) \cdot u_{0}(x, h)$.

ДокАЗАТЕЛьство. Рассмотрим случай $r \geqslant 0$ (при $r=0$ утверждение очевидно). Согласно [2; предложение 4.1] норма пространства $M_{1}(r, h)$ эквивалентна норме $M(r, h)$ при $0 \leqslant r<1$ :

$$
\begin{aligned}
\|\nu(x, h)\|_{M_{1}(r, h)=}^{2}= & \Omega_{h} \sum_{|l| \neq 0} \frac{1}{\left.|l|\right|^{n+2 r} \cdot \Omega_{h}^{1+2 r / n}} \cdot \Omega_{h} \sum_{k \in \mathbb{Z}_{n}}|\nu(k+l)-\nu(k)|^{2} \\
& +\Omega_{h} \sum_{k \in \mathbb{Z}_{n}}|\nu(k)|^{2} \\
= & I_{1}(r, h)+I_{2}(r, h),
\end{aligned}
$$

где $\Omega_{h}=\prod_{j=1}^{n} h_{j}, \nu(k)=\pi \nu(x, h)$.

Так как семейство функций $u_{0}(x, h)$ липшицево, то справедлива оценка

$$
I_{2}(r, h) \leqslant C_{1}\|u(x, h)\|_{M(r, h)}^{2},
$$

где $C_{1}$ не зависит от $h=\left(h_{1}, \ldots, h_{n}\right)$.

Имеет место элементарное неравенство

$$
|\nu(k+l)-\nu(k)|^{2} \leqslant A\left\{\left|u_{0}(k+l)\right|^{2} \cdot|u(k+l)-u(k)|^{2}+|u(k)|^{2} \cdot\left|u_{0}(k+l)-u_{0}(k)\right|^{2}\right\} .
$$

Используя (6.4), получаем неравенство

$$
\begin{aligned}
I_{1}(r, h) \leqslant & A \Omega_{h} \sum_{|l| \neq 0} \frac{1}{|l| n+2 r \cdot \Omega_{h}^{1+2 r / n}} \cdot \Omega_{h} \sum_{k \in \mathbb{Z}_{n}}\left|u_{0}(k+l)\right|^{2}|u(k+l)-u(k)|^{2} \\
& +A \Omega_{h} \sum_{|l| \neq 0} \frac{1}{|l|^{n+2 r} \cdot \Omega_{h}^{1+2 r / n}} \cdot \Omega_{h} \sum_{k \in \mathbb{Z}_{n}}|u(k)|^{2} \cdot\left|u_{0}(k+l)-u_{0}(k)\right|^{2} \\
= & B_{1}(r, h)+B_{2}(r, h) .
\end{aligned}
$$


Из липшицевости семейства $u_{0}(x, h)$ следует:

$$
B_{1}(r, h) \leqslant C_{2}(r)\|u(x, h)\|_{M(r, h)}^{2}
$$

где $C_{2}(r)$ не зависит от $h$.

Пусть $h_{0}=\min h_{j} ;$ если $h_{0} \geqslant 1$, то вследствие того что в $I_{1}(r, h)$ ряд абсолютно сходится, неравенство (6.1) будет справедливо.

Пусть теперь $0<h_{0}<1$, тогда из неравенства $(2.3)$ следует: существует число $\delta>0$, не зависящее от $h$, такое, что $|h|<\delta$. Возьмем число $L=10 \delta$. Обозначим через $\Lambda_{1}(h)$ множество всех мультииндексов $l$, для которых вьполняется $|l| \neq 0$, $\left(\sum_{i=1}^{n}\left(l_{i} h_{i}\right)^{2}\right)^{1 / 2}<L$, через $\Lambda_{2}(h)$ - множество всех остальных мультииндексов $l$, $|l| \neq 0$. Запишем $B_{2}(r, h)$ в виде

$$
\begin{aligned}
B_{2}(r, h) & =\sum_{i=1}^{2} A \Omega_{h} \sum_{l \in \Lambda_{i}(h)} \frac{1}{|l|^{n+2 r} \cdot \Omega_{h}^{1+2 r / n}} \cdot \Omega_{h} \sum_{k \in \mathbb{Z}_{n}}|u(k)|^{2} \cdot\left|u_{0}(k+l)-u_{0}(k)\right|^{2} \\
& =C_{1}(r, h)+C_{2}(r, h) .
\end{aligned}
$$

Используя липшицевость семейства $u_{0}(x, h)$, из $(6.7)$ получаем

$$
\begin{aligned}
C_{2}(r, h) & \leqslant A_{1}(r) \Omega_{h} \sum_{l \in \Lambda_{2}(h)} \frac{\|u(x, h)\|_{L_{2}\left(\mathbb{R}_{n}\right)}^{2}}{(|l||h|)^{n+2 r}} \\
& \leqslant A_{2}(r) \int_{9 \delta}^{\infty} \frac{d \rho}{\rho^{1+2 r}} \cdot\|u(x, h)\|_{L_{2}\left(\mathbb{R}_{n}\right)}^{2} \\
& \leqslant A_{3}(r)\|u(x, h)\|_{L_{2}\left(\mathbb{R}_{n}\right)}^{2}, \\
C_{1}(r, h) & \leqslant A_{4}(r) \Omega_{h} \sum_{l \in \Lambda_{1}(h)} \frac{\|u(x, h)\|_{L_{2}\left(\mathbb{R}_{n}\right)}^{2} \cdot|l|^{2} \cdot|h|^{2}}{(|l||h|)^{n+2 r}} \\
& \leqslant A_{5}(r) \int_{0}^{11 \delta} \frac{d \rho}{\rho^{-1+2 r}} \cdot\|u(x, h)\|_{L_{2}\left(\mathbb{R}_{n}\right)}^{2} \\
& \leqslant A_{6}(r)\|u(x, h)\|_{L_{2}\left(\mathbb{R}_{n}\right)}^{2} \cdot
\end{aligned}
$$

Из (6.3), (6.5)-(6.9) следует неравенство (6.1) при $r>0$.

Докажем неравенство (6.1) при $-1<r<0$.

Рассмотрим множество линейных функционалов $I_{\lambda}$ над $M(r, h)$, определяемых функциями $\lambda(x, h) \in M(-r, h), 0<-r<1,\|\lambda(x, h)\|_{M(-r, h)}=1$. Для доказательства (6.1) достаточно установить неравенство

$$
\left|I_{\lambda}(\nu(x, h))\right| \leqslant C(r)\|u(x, h)\|_{M(r, h)},
$$

где $C(r)$ не зависит от $h$ и $\lambda(x, h)$. Имеем:

$$
I_{\lambda}(\nu(x, h))=\int_{\mathbb{R}_{n}} u_{0}(x, h) u(x, h) \cdot \overline{\lambda(x, h)} d x .
$$

Так как $0<-r<1$, то к семейству $b(x, h)=u_{0}(x, h) \overline{\lambda(x, h)}$ применимо неравенство (6.1). Из последнего замечания и (6.11) следует (6.10).

Предложение 6.1. доказано. 
6.1. Задача Дирихле для общего эллиптического уравнения 2-го порядка. В дальнейшем будем предполагать, что $\Omega$ - открытая, ограниченная область с липшицевой границей, нормальная, допускающая разбиение (см. [2; определения 6.1-6.4, 6.6, 6.9]). Кроме того, будем считать, что область $\Omega$ такова, что любая функция $a(x) \in C^{1}(\bar{\Omega})$ допускает продолжение $\widetilde{a}(x) \in C^{1}\left(\mathbb{R}_{n}\right)$.

Рассмотрим равномерно эллиптическое уравнение

$$
\sum_{i=1}^{n} \frac{\partial}{\partial x_{i}} \sum_{j=1}^{n} a_{i j}(x) \frac{\partial u}{\partial x_{j}}=f(x)
$$

где $a_{i j}(x) \in C^{1}(\bar{\Omega}), u(x) \in \stackrel{\circ}{H}_{1}(\Omega), f(x) \in H_{-1}(\Omega), a_{i j}(x)=a_{j i}(x)$ и имеет место неравенство (5.22). Для нахождения приближенного решения $u(x, h)$ уравнения (6.12) рассмотрим разностное уравнение

$$
\mathrm{L}_{h} u(x, h)=f(x, h),
$$

где $\mathrm{L}_{h}$ - семейство операторов, определенных соотношением $(5.23), u(x, h) \in$ $\stackrel{\circ}{M}\left(1, h, \Omega_{1 h}\right), f(x, h) \in M\left(-1, h, \Omega_{1 h}\right)$, причем существует продолжение $\widetilde{f}(x, h) \in$ $M(-1, h)$ функции $f(x, h)$, которое сходится по норме $H_{-1}\left(\mathbb{R}_{n}\right)$ при $|h| \rightarrow 0$ к $l f(x) \in H_{-1}\left(\mathbb{R}_{n}\right)$, где $l$ - некоторое продолжение $f(x)$.

Из предложения 5.5 следует, что ограниченное семейство операторов $\mathrm{P}_{h} \mathrm{~L}_{h}$ устойчиво отображает $\stackrel{\circ}{M}\left(1, h, \Omega_{1 h}\right)$ в $M\left(-1, h, \Omega_{1 h}\right), \mathrm{P}_{h}$ - оператор сужения на $\Omega_{1 h}$. Из предложения 6.1 и условий на $\Omega$ следует, что семейство операторов $\mathrm{L}_{h}$ ограниченно отображает $\stackrel{\circ}{M}\left(1, h, \Omega_{1 h}\right), 0<r \leqslant 1$, в $M(r-2, h)$. Отсюда следует, что для того чтобы воспользоваться предложением 2.3 , достаточно установить условие $\prod_{h}^{*}$-аппроксимации (см. определение 1.7$)$. Нужно заметить, что оператор

$$
\mathrm{L}(u)=\sum_{i=1}^{n} \frac{\partial}{\partial x_{i}} \sum_{j=1}^{n} a_{i j}(x) \frac{\partial u}{\partial x_{j}}
$$

ограничен из $\stackrel{\circ}{H}_{1}(\Omega)$ в $H_{-1}(\Omega)$.

Пусть $\varphi(x) \in C_{0}^{\infty}(\Omega)$, тогда $\mathrm{L}(\varphi) \in C(\bar{\Omega})$, т.е. оператор $\mathrm{L}(u)$ - конечного порядка (см. определение 1.3). Так как $\varphi(x) \in C_{0}^{\infty}(\Omega)$, то существует $\delta>0$ такое, что $\operatorname{supp}_{h} \prod_{h}^{*} \varphi(x) \subset \Omega_{1 h}$ при $|h|<\delta$ (см. [2; определение 5.2]). Введем обозначение: $\psi_{j}\left(k_{1} h_{1}, \ldots, k_{n} h_{n}\right)=\pi \mathrm{T}_{\text {пр }}\left(x_{j}\right) \prod_{h}^{*} \varphi(x)$ (см. [2; определение 1.2]). Тогда имеет место соотношение

$$
\begin{aligned}
\pi \mathrm{T}_{\text {лев }}\left(x_{i}\right)\left[a_{i j}(x, h) \psi_{j}(x, h)\right] & \\
= & \frac{a_{i j}\left(k_{1} h_{1}, \ldots, k_{i} h_{i}, \ldots, k_{n} h_{n}\right)-a_{i j}\left(k_{1} h_{1}, \ldots,\left(k_{i}-1\right) h_{i}, \ldots, k_{n} h_{n}\right)}{h_{i}} \\
& \times \psi_{j}\left(k_{1} h_{1}, \ldots, k_{n} h_{n}\right)+a_{i j}\left(k_{1} h_{1}, \ldots,\left(k_{i}-1\right) h_{i}, \ldots, k_{n} h_{n}\right) \\
& \times \frac{\psi_{j}\left(k_{1} h_{1}, \ldots, k_{i} h_{i}, \ldots, k_{n} h_{n}\right)-\psi_{j}\left(k_{1} h_{1}, \ldots,\left(k_{i}-1\right) h_{i}, \ldots, k_{n} h_{n}\right)}{h_{i}} .
\end{aligned}
$$


Используя (6.14), при помощи условий $a_{i j}(x) \in C^{1}(\bar{\Omega})$ получаем соотношение

$$
\left|\mathrm{L} \varphi\left(k_{1} h_{1}, \ldots, k_{n} h_{n}\right)-\pi \mathrm{L}_{h} \prod_{h}^{*} \varphi(x)\right|=O(|h|),
$$

где $O(|h|) \rightarrow 0$ при $|h| \rightarrow 0$ равномерно для всех $k$ таких, что $D(k, h) \in \Omega_{1 h}$. Так как $\mathrm{L}(\varphi) \in C(\bar{\Omega})$, то из $(6.15)$ следует, что операторы $\mathrm{L}_{h} \prod_{h}^{*}$-аппроксимируют оператор L в области $\Omega$.

Все условия предложения 2.3 выполняются. Отсюда следует, что решение уравнения (6.12) сушествует и единственно для любой $f(x) \in H_{-1}(\Omega)$. Решения $u(x, h)$ уравнения (6.13) сильно сходятся в $\stackrel{\circ}{H}_{r}(\Omega)$ для любого $r, 0<r<\frac{1}{2}$, к истинному решению $u(x) \in \stackrel{\circ}{H}_{1}(\Omega)$ и, кроме того, $\left\|u(x, h)-\prod_{1 h} u(x)\right\|_{M\left(1, h, \Omega_{1 h}\right)} \rightarrow 0$ при $|h| \rightarrow 0$, где $\prod_{1 h}-$ сужение интегрального проектора $\prod_{h}$ на область $\Omega_{1 h}$.

Рассмотрим оператор

$$
\mathrm{M}(u)=\mathrm{L}(u)+\mathrm{F}(u)=\sum_{i=1}^{n} \frac{\partial}{\partial x_{i}} \sum_{j=1}^{n} a_{i j}(x) \frac{\partial u}{\partial x_{j}}+\sum_{i=1}^{n} b_{i}(x) \frac{\partial u}{\partial x_{i}}+\lambda(x) u(x)
$$

где $b_{i}(x), \lambda(x) \in C(\bar{\Omega}) ; a_{i j}(x)=a_{j i}(x) \in C^{1}(\bar{\Omega})$; для оператора $\mathrm{L}(u)$ выполнено (5.12).

Рассмотрим уравнение

$$
\mathrm{M}(u)=f,
$$

где $u(x) \in \stackrel{\circ}{H}_{1}(\Omega), f(x) \in H_{-1}(\Omega)$; пусть ядро оператора М состоит из одного нулевого элемента.

Для нахождения приближенного решения $u(x, h)$ уравнения $(6.16)$ рассмотрим разностное уравнение

$$
\mathrm{M}_{h} u(x, h)=f(x, h),
$$

где $\mathrm{M}_{h}=\mathrm{L}_{h}+\mathrm{F}_{h}, \mathrm{~F}_{h}=\sum_{i=1}^{n} b_{i}(x, h) \mathrm{T}_{\text {пр }}\left(x_{i}\right) u(x, h)+\lambda(x, h) u(x, h), \pi b_{i}(x, h)=$ $b_{i}\left(k_{1} h_{1}, \ldots, k_{n} h_{n}\right), \pi \lambda(x, h)=\lambda\left(k_{1} h_{1}, \ldots, k_{n} h_{n}\right), u(x, h) \in \stackrel{\circ}{M}\left(1, h, \Omega_{1 h}\right), f(x, h) \in$ $M\left(-1, h, \Omega_{1 h}\right)$, причем сушествуют продолжения $\widetilde{f}(x, h) \in M(-1, h)$ функций $f(x, h)$, которые сходятся к $l f(x) \in H_{-1}\left(\mathbb{R}_{n}\right)$ по норме $H_{-1}\left(\mathbb{R}_{n}\right)$ при $|h| \rightarrow 0$, где lf - некоторое продолжение $f(x)$.

Аналогично, как и вьше, проверяется, что выполняются все условия предложения 2.5. Отсюда следует, что решение $u(x) \in \stackrel{\circ}{H}_{1}(\Omega)$ уравнения (6.16) сушествует и единственно $\forall f(x) \in H_{-1}(\Omega)$. Кроме того, найдется такое число $\delta>0$, что при $|h|<\delta$ сушествуют решения $u(x, h)$ уравнения $(6.17)$, которые сильно сходятся в $\stackrel{\circ}{H}_{r}(\Omega)$ при любом $r, 0<r<\frac{1}{2}$, к истинному решению $u(x) \in \stackrel{\circ}{H}_{1}(\Omega)$, причем $\left\|u(x, h)-\prod_{1 h} u(x)\right\|_{\stackrel{\circ}{\circ}\left(1, h, \Omega_{1 h}\right)}^{\rightarrow} 0$ при $|h| \rightarrow 0$.

\section{2. Характеристическое гиперсингулярное уравнение}

$$
\int_{\Omega} \frac{u(x) d x}{r^{n+2 p+\lambda}}=f\left(x_{0}\right)
$$

где $0<\lambda<2, p=0,1,2, \ldots, u(x) \in \stackrel{\circ}{H}_{p+\lambda / 2}(\Omega), f\left(x_{0}\right) \in H_{-p-\lambda / 2}(\Omega)$. 
Определение символа в левой части (6.18) задано в (4.32).

Оператор Т $u=\int_{\Omega} \frac{u(x) d x}{r^{n+2 p+\lambda}}$ ограничен из $\stackrel{\circ}{H}_{p+\lambda / 2}(\Omega)$ в $H_{-p-\lambda / 2}(\Omega)$ (cм. [3]).

Для нахождения приближенного решения $u(x, h)$ уравнения $(6.18)$ рассмотрим уравнение

$$
C(\lambda, p) \mathrm{T}_{h}(\lambda, p) u(x, h)=f(x, h)
$$

где операторы $\mathrm{T}_{h}(\lambda, p)$ определены соотношениями $(5.1)$ и $(3.50), C(\lambda, 0)=1$, $u(x, h) \in \stackrel{\circ}{M}\left(p+\lambda / 2, h, \Omega_{1 h}\right), f(x, h) \in M\left(-p-\lambda / 2, h, \Omega_{1 h}\right)$, причем существуют продолжения $\widetilde{f}(x, h) \in M(-p-\lambda / 2, h)$ функций $f(x, h)$, которые сходятся к $l f(x) \in H_{-p-\lambda / 2}\left(\mathbb{R}_{n}\right)$ по норме $H_{-p-\lambda / 2}\left(\mathbb{R}_{n}\right)$ при $|h| \rightarrow 0$, где $l f-$ некоторое продолжение $f(x)$.

Из предложений $3.4,4.1,4.3$, пп. 3.4, 5.2 и 5.3 следует, что для оператора Т и семейства операторов $C(\lambda, p) \mathrm{T}_{h}(\lambda, p)$ выполняются все условия предложения 2.3. Отсюда получаем, что решение $u(x) \in \stackrel{\circ}{H}_{p+\lambda / 2}(\Omega)$ уравнения $(6.18)$ существует и единственно для любой $f(x) \in H_{-p-\lambda / 2}(\Omega)$. Если $p=0,0<\lambda<1$, то решения $u(x, h)$ уравнения (6.19) сходятся к $u(x) \in \stackrel{\circ}{H}_{\lambda / 2}(\Omega)$ по норме $\stackrel{\circ}{H}_{r}(\Omega), 0<r<\lambda / 2$, при $|h| \rightarrow 0$; если $p=0,1 \leqslant \lambda<2$, то $u(x, h)$ сходятся к $u(x) \in \stackrel{\circ}{H}_{\lambda / 2}(\Omega)$ по норме $\stackrel{\circ}{H}_{r}(\Omega)$ для любого $r, 0<r<\frac{1}{2}$; если $p=1,2, \ldots, 0<\lambda<2$, то $u(x, h)$ сходятся к $u(x) \in \stackrel{\circ}{H}_{p+\lambda / 2}(\Omega)$ по норме $\stackrel{\circ}{H}_{r}(\Omega)$ для любого $r, 0<r<\frac{1}{2}$, при $|h| \rightarrow 0$. Во всех случаях при $|h| \rightarrow 0$

$$
\left\|u(x, h)-\prod_{1 h} u(x)\right\|_{\stackrel{\circ}{M\left(p+\lambda / 2, h, \Omega_{1 h}\right)}} \rightarrow 0
$$

6.3. Итерированный оператор Лапласа порядка $m$ (задача Дирихле). Рассмотрим уравнение

$$
\Delta^{m} u(x)=f(x)
$$

где $\Delta^{m}$ - итерированный оператор Лапласа порядка $m, u(x) \in \stackrel{\circ}{H}_{m}(\Omega)$, $f(x) \in H_{-m}(\Omega)$.

Приближенное решение $u(x, h)$ уравнения $(6.20)$ ищем из уравнения

$$
\Delta_{h p}^{m} u(x, h)=f(x, h)
$$

где $\Delta_{h p}^{m}$ - разностный оператор Лапласа порядка $m, u(x, h) \in \stackrel{\circ}{M}\left(m, h, \Omega_{1 h}\right)$, $f(x, h) \in M\left(-m, h, \Omega_{1 h}\right) ;$ причем существуют продолжения $\widetilde{f}(x, h) \in M(-m, h)$ функций $f(x, h)$, которые сходятся к $l f(x) \in H_{-m}\left(\mathbb{R}_{n}\right)$ по норме $H_{-m}\left(\mathbb{R}_{n}\right)$ при $|h| \rightarrow 0$, где $l f$ - некоторое продолжение $f(x)$.

Из [2; предложения 5.3 и 5.8] и п. 3.4.б) вытекает, что все условия предложения 2.3 выполнены. Отсюда следует, что решение $u(x) \in \stackrel{\circ}{H}_{m}(\Omega)$ уравнения (6.20) существует и единственно для любой $f(x) \in H_{-m}(\Omega)$. Приближенные решения $u(x, h)$ уравнения $(6.21)$ сходятся к $u(x) \in \stackrel{\circ}{H}_{m}(\Omega)$ по норме $\stackrel{\circ}{H}_{r}(\Omega)$ для любого $r$,

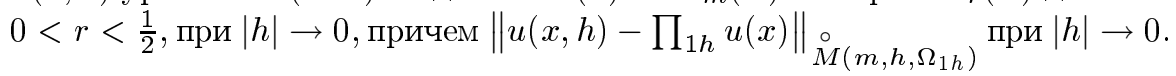


6.4. Уравнение из задач дифракции. Пусть функция $u(x)$ удовлетворяет уравнению Гельмгольца:

$$
\begin{aligned}
& \Delta u+\lambda^{2} u=0, \quad \quad x \in \mathbb{R}_{3} \backslash \Omega \\
&\left.\frac{d u}{d n}\right|_{\Omega}=f,
\end{aligned}
$$

где $\Delta$ - оператор Лапласа, $\Omega$ - ограниченный кусок некоторой плоскости $\alpha, n$ - нормаль к $\Omega ; f$ - заданная функция на $\Omega$. Область $\Omega$ нормальна и допускает разбиение (см. [2; определения 6.6, 6.9]).

Пусть выполняются условия излучения Зоммерфельда:

$$
u(x)=O\left(|x|^{-1}\right), \quad \frac{\partial u(x)}{\partial|x|}+i \lambda u(x)=o\left(|x|^{-1}\right)
$$

при $|x| \rightarrow \infty$.

Кроме того, будем предполагать, что

$$
\operatorname{Re} \lambda>0, \quad \operatorname{Im} \lambda \leqslant 0 .
$$

$\Phi$ ундаментальное решение уравнения $\Delta u+\lambda^{2} u=0$ с условиями $(6.23)$ в $\mathbb{R}_{3}$ имеет вид

$$
\varepsilon=-\frac{1}{4 \pi r}-\frac{1}{4 \pi} \frac{e^{-i \lambda r}-1}{r}
$$

Решение задачи (6.22) с условиями (6.23) будем искать через потенциал двойного слоя $u(x, y)$, распределенный на $\Omega$. Тогда относительно функции $u(x, y)$ имеет место уравнение

$$
\mathrm{T}(u)+\mathrm{F}(u)=g\left(M_{0}\right),
$$

где $\mathrm{T}(u)=\int_{\Omega} \frac{u(x, y) d x d y}{r_{M M_{0}}^{3}} ; \mathrm{F}(u)=\int_{\Omega} \frac{J\left(r_{M_{0} M}\right) d x d y}{r_{M M_{0}}^{3}} ; g\left(M_{0}\right)=-4 \pi f\left(x_{0}, y_{0}\right)$, $r_{M M_{0}}=\sqrt{\left(x_{0}-x\right)^{2}+\left(y_{0}-y\right)^{2}} ; J\left(r_{M_{0} M}\right)=e^{-i \lambda r_{M M_{0}}}+i \lambda e^{-i \lambda r_{M M_{0}} r_{M M_{0}}-1,}$ $u(x, y) \in \stackrel{\circ}{H}_{1 / 2}(\Omega), g\left(x_{0}, y_{0}\right) \in H_{-1 / 2}(\Omega)$.

Пусть число $\lambda$ таково, что ядро оператора $\mathrm{M}(u)=\mathrm{T}(u)+\mathrm{F}(u)$ состоит из одного нулевого элемента.

Так как $\Omega$ - ограниченная область, то существуют ограниченная область $\Omega_{1}>\Omega$ и функция $\alpha\left(O M_{0}\right) \in C_{0}^{\infty}\left(\mathbb{R}_{2}\right)\left(O\right.$ - начало координат), $\alpha\left(x_{0}, y_{0}\right)=1$ при $M_{0}\left(x_{0}, y_{0}\right) \in \Omega_{1}$, такие, что уравнение

$$
\int_{\Omega} \frac{u(x, y)}{r_{M M_{0}}^{3}} d x d y+\int_{\Omega} \frac{J_{1}\left(r_{M M_{0}}\right) u(x, y)}{r_{M M_{0}}^{3}} d x d y=g\left(M_{0}\right)
$$

будет эквивалентно уравнению $(6.26)$, где $J_{1}\left(r_{M M_{0}}\right)=J\left(r_{M M_{0}}\right) \cdot \alpha\left(r_{M M_{0}}\right)$, $\alpha\left(r_{M M_{0}}\right)=1$ при $M, M_{0} \in \Omega$.

Имеет место неравенство

$$
\left|J_{2}\left(r_{M M_{0}}\right)\right|=\left|\frac{J_{1}\left(r_{M M_{0}}\right)}{r_{M M_{0}}^{3}}\right|<\frac{C}{r_{M M_{0}}},
$$


отсюда $J_{2}\left(r_{M M_{0}}\right) \in L\left(\mathbb{R}_{2}\right)$ при каждом фиксированном $M_{0}$. Уравнение (6.27) запишем в операторной форме

$$
\mathrm{T}(u)+\mathrm{F}_{1}(u)=g\left(M_{0}\right) .
$$

Для нахождения приближенного решения $u(x, h)$ уравнения (6.28) разобъем плоскость $\mathbb{R}_{2}$ с векторньм шагом $h=\left(h_{1}, h_{2}\right)$ на ячейки $D(k, h)$ (см. [2; определение 1.1]), причем для разбиения выполняется условие (2.3) из [2]. Сеточную функцию $u(k)=\pi u(x, h)$ (см. [2; определение 1.2]) будем находить из уравнения

$$
\sum_{k} u(k) \gamma_{1}(k, m)+\sum_{k} u(k) \gamma_{2}(k, m)=g\left(M_{0 m}\right), \quad M_{0 m} \in \Omega_{1 h},
$$

где суммирование происходит по всем номерам $k=\left(k_{1}, k_{2}\right)$ таким, что $D(k, h) \in$ $\Omega_{1 h} ; M_{0 m}=M_{0 m}\left(m_{1} h_{1}, m_{2} h_{2}\right) ; \pi g(x, h)=g\left(M_{0 m}\right)$, причем сушествуют продолжения $\widetilde{g}(x, h) \in M(-1 / 2, h)$ функций $g(x, h)$, которые сходятся к $l g(x) \in H_{-1 / 2}\left(\mathbb{R}_{n}\right)$ при $|h| \rightarrow 0, \lg (x)$ - некоторое продолжение $g(x)$. Коэффициенты уравнений (6.29) определяются из соотношений

$$
\gamma_{1}(k, m)=\int_{D(k, h)} \frac{d x d y}{r_{M M_{0 m}}^{3}} ; \quad \gamma_{2}(k, m)=\int_{D(k, h)} J_{2}\left(r_{M M_{0 m}}\right) d x d y .
$$

Оператор $\mathrm{T}_{h 1}=\sum_{k} u(k) \gamma_{1}(k, m)$ является вихревым. Относительно $\mathrm{T}_{h 1}$ справедливы все условия (как было доказано ранее) предложения 2.3. Дискретньй оператор $\mathrm{F}_{1 h} u(k)=\sum_{k} u(k) \gamma_{2}(k, m)$ представляет собой оператор дискретной свертки. Для символа $\widehat{F}_{1 h}(\varphi)$ этого оператора (см. [2; формула (1.9)]) имеет место неравенство

$$
\left|\widehat{\mathrm{F}}_{1 h}(\varphi)\right|=\left|\sum_{k} \gamma_{2}(k, 0) e^{i(k, \varphi)}\right| \leqslant \int_{\mathbb{R}_{2}}\left|J_{2}\left(r_{M M_{0 m}}\right)\right| d x d y \leqslant C
$$

Из (6.31) следует: семейство операторов $\mathrm{F}_{1 h}$ ограниченно отображает пространства $M(r, h)$ в $M(r, h)$ для любого $r$. Так как имеет место неравенство

$$
\sum_{k}\left|\gamma_{2}(k, m)\right| \leqslant C_{1}
$$

где $C_{1}$ не зависит от $m$ и $h$, то для оператора $\mathrm{F}_{1}$ и семейства $\mathrm{F}_{1 h}$ будет выполняться условие $\prod_{h}^{*}$-аппроксимации в области $\Omega$. Итак, для операторов $\mathrm{T}, \mathrm{F}_{1}$ и семейств операторов $\mathrm{T}_{h 1}$ и $\mathrm{F}_{h 1}$ выполняются все условия предложения 2.5 из [2]. Отсюда следует, что решение $u(x, y) \in \stackrel{\circ}{H}_{1 / 2}(\Omega)$ уравнений $(6.26),(6.28)$ сушествует и единственно. Найдется $\delta>0$ такое, что при $|h|<\delta$ система уравнений (6.29) разрешима. Решения $u(x, h)$ системы $(6.29)$ сходятся к $u(x) \in H_{1 / 2}(\Omega)$ по норме $\stackrel{\circ}{H}_{r}(\Omega)$ для любого $r, 0<r<\frac{1}{2}$, при $|h| \rightarrow 0$. Кроме того, имеет место $\| u(x, h)-$

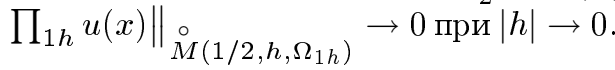




\section{§ 7. Бесциркулярное обтекание поверхности}

В аэродинамике при бесциркуляционном обтекании поверхности $\sigma$ получается уравнение относительно плотности диполей $\nu(M)$, распределенной по поверхности $\sigma$ :

$$
\int_{\sigma} \frac{\nu(M)}{\left|M M_{0}\right|^{3}}\left[\frac{\left|M M_{0}\right|^{2}\left(\mathbf{n}_{M_{0}}, \mathbf{n}_{M}\right)-3\left(\mathbf{M M}_{0}, \mathbf{n}_{M}\right)\left(\mathbf{M M}_{0}, \mathbf{n}_{M_{0}}\right)}{\left|M M_{0}\right|^{2}}\right] d \sigma_{M}=g\left(M_{0}\right),
$$

где $M, M_{0} \in \sigma ; \mathbf{n}_{M_{0}}, \mathbf{n}_{M}$ - орты нормалей к поверхности $\sigma$ в точках $M$ и $M_{0}$ соответственно; $g\left(M_{0}\right)$ - известная функция.

Будем предполагать, что сушествуют поверхности $\sigma_{1}$ и $\sigma_{2}$ такие, что $\sigma \subset \sigma_{1} \subset \sigma_{2}$. Найдется такая декартова система координат $O X Y Z$, в которой поверхность $\sigma_{2}$ задается уравнением $z=f(x, y)$. Пусть $\Omega, \Phi$ и $\Lambda$ - проекции $\sigma, \sigma_{1}$ и $\sigma_{2}$ на плоскость $X O Y$, тогда $f(x, y) \in C^{4}(\bar{\Lambda})$, причем $Г \Phi$ и $Г \Lambda$, границы областей $\Phi$ и $\Lambda$, находятся на положительном расстоянии от $\Omega$ и $\Phi$ соответственно. Область $\Lambda$ такова, что она допускает продолжение $l f \in C^{4}\left(\mathbb{R}_{2}\right)$ любой функции $f(x, y) \in C^{4}(\bar{\Lambda})$. Границу $\Gamma \Lambda=L$ области $\sigma_{2}$ будем считать вьпуклой [5], т.е. для нее выполняются условия: выбран обход на $L$ так, что если смотреть с конца любого вектора $\mathbf{n}_{M}$, то он (обход) виден против часовой стрелки; тройка векторов $\mathbf{n}_{M_{0}}, \mathbf{M M}_{0}, \boldsymbol{\tau}_{M}$ является правой для любых $M_{0} \in \sigma_{2}, M \in L$, где $\tau_{M}$ - касательный вектор к $L$, направление которого совпадает с направлением обхода $L$.

Итак, имеет место

$$
\left(\mathbf{n}_{M_{0}}, \mathbf{M M}_{0}, \tau_{M}\right)>0 .
$$

На поверхности $\sigma_{2}$ выбрано непрерьвное семейство нормалей. Из условия (7.2) для любой $M_{0} \in \sigma_{2}\left(M \in \sigma_{2}\right)$ вытекает неравенство

$$
\int_{\sigma_{2}} G\left(M, M_{0}\right) d \sigma_{M}<0 \quad\left(\int_{\sigma_{2}} G\left(M, M_{0}\right) d \sigma_{M_{0}}<0\right)
$$

где $G\left(M, M_{0}\right)=G\left(M_{0}, M\right)$ - ядро интеграла в (7.1).

Пусть для любых $M, M_{0} \in \sigma_{2}$ выполняется неравенство

$$
\begin{aligned}
0<C_{1} & <G_{2}\left(M, M_{0}\right) \\
& =\frac{\left|M_{0} M\right|^{2}\left(\mathbf{n}_{M_{0}}, \mathbf{n}_{M}\right)-3\left(\mathbf{M M}_{0}, \mathbf{n}_{M}\right)\left(\mathbf{M M}_{0}, \mathbf{n}_{M_{0}}\right)}{\left|M_{0} M\right|^{2}}<C_{2} .
\end{aligned}
$$

Из сделанных предположений вытекает, что существует продолжение $l f \in$ $C^{4}\left(\mathbb{R}_{2}\right)$ функции $z=f(x, y) \in C^{4}(\bar{\Lambda})$. Без ограничения общности можно считать, что $l f$ - финитная функция; оставим для $l f$ прежнее обозначение $f(x, y)$.

Разобьем плоскость $X O Y$ с векторным шагом $h=(h, h)$ (шаг разбиения $h>0$ на каждой оси одинаков) на элементарные ячейки $D(k, h)($ см. [2; определение 1.1]). Обозначим через $a(k, m)$ величину

$$
a(k, m)=\iint_{D(m, h)} \frac{d x d y}{\left[\left(x-h m_{1}\right)^{2}+\left(y-h m_{2}\right)^{2}+\left(f(x, y)-f\left(h m_{1}, h m_{2}\right)\right)^{2}\right]^{3 / 2}}
$$


ПРЕДЛОЖЕНИЕ 7.1. При сделанных выше предположениях имеет место неравенство

$$
\Delta a(k, m)=|a(k, m)-a(m, k)| \leqslant C h \gamma^{*}(k, m)
$$

əде $C$ не зависит от $h, k u m$,

$$
\gamma^{*}(k, m)=\iint_{D(m, h)} \frac{d x d y}{\left[\left(x-h m_{1}\right)^{2}+\left(y-h m_{2}\right)^{2}\right]^{3 / 2}}, \quad k \neq m .
$$

ДокАЗАТЕльство. Так как по условию $k \neq m$, то в интеграле (7.5) особенности нет. Сделаем в (7.5) замену: $x-h m_{1}=h k_{1}-z_{0}, y-h m_{2}=h k_{2}-t_{0}$, тогда получим

$$
\begin{aligned}
a(k, m)= & \iint_{D(m, h)}\left[\left(x-k_{1} h\right)^{2}+\left(y-k_{2} h\right)^{2}\right. \\
& \left.+\left(f\left(h\left(k_{1}+m_{1}\right)-x, h\left(k_{2}+m_{2}\right)-y\right)-f\left(h m_{1}, h m_{2}\right)\right)^{2}\right]^{-3 / 2} d x d y .
\end{aligned}
$$

Используя тот факт, что функция $g(z)=(1+z)^{-3 / 2}$ имеет ограниченную производную при $z \geqslant 0, z=\left(\frac{f(x, y)-f\left(x_{0}, y_{0}\right)}{r\left(x_{0}, y_{0}\right)}\right)^{2}, r\left(x_{0}, y_{0}\right)=\sqrt{\left(x-x_{0}\right)^{2}+\left(y-y_{0}\right)^{2}}$,

$$
\Delta a(k, m)=\left|\iint_{D(m, h)} \frac{1}{r_{k_{1} k_{2}}^{3}} g^{\prime}\left(z_{1}\right) \Delta z d x d y\right|
$$

где $r_{k_{1} k_{2}}=\sqrt{\left(x-h k_{1}\right)^{2}+\left(y-h k_{2}\right)^{2}},(x, y) \in D(m, h) ; z_{1}>0$ - некоторое число.

Оценим величину $\Delta z$ :

$$
\begin{aligned}
|\Delta z| \leqslant C \mid & \frac{f(x, y)-f\left(k_{1} h, k_{2} h\right)}{r_{k_{1} k_{2}}} \\
& -\frac{f\left(h m_{1}, h m_{2}\right)-f\left(h\left(k_{1}+m_{1}\right)-x, h\left(k_{2}+m_{2}\right)-y\right)}{r_{k_{1} k_{2}}} \mid .
\end{aligned}
$$

Здесь было использовано неравенство

$$
\left|\frac{f(x, y)-f\left(k_{1} h, k_{2} h\right)}{r_{k_{1} k_{2}}}+\frac{f\left(h m_{1}, h m_{2}\right)-f\left(h\left(k_{1}+m_{1}\right)-x, h\left(k_{2}+m_{2}\right)-y\right)}{r_{k_{1} k_{2}}}\right|<C,
$$

где $(x, y) \in D(m, h),\left(k_{1}, k_{2}\right) \neq\left(m_{1}, m_{2}\right)$.

Разлагая функции, находящиеся в правой части (7.8), по формуле Тейлора в точке $\left(h m_{1}, h m_{2}\right)$, получаем

$$
\begin{aligned}
|\Delta z| \leqslant & C \mid \frac{f_{x}^{\prime}\left(h m_{1}, h m_{2}\right)-f_{x}^{\prime}\left(h k_{1}, h k_{2}\right)}{r_{k_{1} k_{2}}}\left(x-h m_{1}\right) \\
& +\frac{f_{y}^{\prime}\left(h m_{1}, h m_{2}\right)-f_{y}^{\prime}\left(h k_{1}, h k_{2}\right)}{r_{k_{1} k_{2}}}\left(y-h m_{2}\right)+\frac{O(h)}{r_{k_{1} k_{2}}} \mid \leqslant C_{1} h
\end{aligned}
$$


где $O(h)$ - остаточный член формулы Тейлора, $|O(h)|<C_{2} h^{2}$ при $(x, y) \in D(m, h)$.

Из (4.7) и (7.9) следует предложение 7.1.

Рассмотрим величины

$$
\begin{aligned}
& \beta_{1}(k, m)=\iint_{D(m, h)} J\left(M_{0 m}^{\prime}, M^{\prime}\right) q(x, y) d x d y \\
& \beta_{2}(m)=\frac{1}{h^{2}} \iint_{D(m, h)} q(x, y) d x d y, \\
& \gamma(k, m)=\beta_{2}(m) \cdot \beta_{1}(k, m) \text {, } \\
& \text { где } q(x, y)=\sqrt{1+\left[f_{x}^{\prime}(x, y)\right]^{2}+\left[f_{y}^{\prime}(x, y)\right]^{2}}, M_{0 m}^{\prime}=M_{0 m}^{\prime}\left(h m_{1}, h m_{2}\right), M^{\prime}=M^{\prime}(x, y) \text {, } \\
& J\left(M_{0}^{\prime}, M^{\prime}\right)=G\left(M_{0}, M\right), M_{0}^{\prime}=M_{0}^{\prime}\left(x_{0}, y_{0}\right) \text {. }
\end{aligned}
$$

ПРЕДЛОЖЕНИЕ 7.2. При $k \neq m$ имеет место неравенство

$$
|\Delta \gamma(k, m)|=|\gamma(k, m)-\gamma(m, k)| \leqslant C h \gamma^{*}(k, m)
$$

әде $C$ не зависит от $h, k u m$.

ДокАЗАТЕЛьство. Введем обозначения:

$$
\begin{aligned}
& G_{1}\left(M, M_{0}\right)=\frac{1}{\left|M M_{0}\right|^{3}}=J_{1}\left(M^{\prime}, M_{0}^{\prime}\right), \\
& G_{2}\left(M, M_{0}\right)=\frac{G\left(M, M_{0}\right)}{G_{1}\left(M, M_{0}\right)}=J_{2}\left(M^{\prime}, M_{0}^{\prime}\right) .
\end{aligned}
$$

Так как $f(x, y) \in C_{0}^{4}\left(\mathbb{R}_{2}\right)$, то функция $J_{2}\left(M^{\prime}, M_{0}^{\prime}\right)$ как функция переменных $x, y$, $x_{0}, y_{0}$ имеет равномерно ограниченные первые производные по этим переменным при $(x, y) \neq\left(x_{0}, y_{0}\right)$, что проверяется непосредственно с использованием формулы Тейлора.

При $k \neq m$ в интегралах, присутствующих в формулах (7.10), особенностей нет, все функции непрерывны и положительны. Отсюда, применяя теорему о среднем, получаем

$$
\begin{aligned}
\gamma(k, m) & =q\left(M_{1}^{\prime}\right) J_{2}\left(M_{0 m}^{\prime}, M_{2}^{\prime}\right) \cdot q\left(M_{3}^{\prime}\right) a(k, m) \\
\gamma(m, k) & =q\left(M_{4}^{\prime}\right) J_{2}\left(M_{0 k}^{\prime}, M_{5}^{\prime}\right) \cdot q\left(M_{6}^{\prime}\right) a(m, k) \\
& =q\left(M_{4}^{\prime}\right) J_{2}\left(M_{5}^{\prime}, M_{0 k}^{\prime}\right) \cdot q\left(M_{6}^{\prime}\right)[a(k, m)+\Delta a(k, m)]
\end{aligned}
$$

где $M_{1}^{\prime}, M_{5}^{\prime}, M_{6}^{\prime} \in D(m, h), M_{2}^{\prime}, M_{3}^{\prime}, M_{4}^{\prime} \in D(k, h)$.

Из сделанного выше замечания относительно функции $J_{2}\left(M^{\prime}, M_{0}^{\prime}\right)=J_{2}\left(M_{0}^{\prime}, M^{\prime}\right)$ и условия (7.4), а также из (7.13) и предложения 7.1 следует неравенство (7.11), что и требовалось доказать.

При $f(x, y) \in C_{0}^{4}\left(\mathbb{R}_{2}\right)$ для функции $Q\left(M, M_{0}\right)$ имеют место оценки

$$
\begin{aligned}
\left|Q\left(M, M_{0}\right)\right| & =\left|\frac{1}{\left|M_{0} M\right|^{3}} \frac{\left|M_{0} M\right|^{2}\left[\left(\mathbf{n}_{M_{0}}, \mathbf{n}_{M}\right)-1\right]-3\left(\mathbf{M}_{0} \mathbf{M}, \mathbf{n}_{M_{0}}\right)\left(\mathbf{M}_{0} \mathbf{M}, \mathbf{n}_{M}\right)}{\left|M_{0} M\right|^{2}}\right| \\
& \leqslant \frac{C}{\left|M_{0} M\right|},
\end{aligned}
$$


$\left|M_{0} M\right| \leqslant \delta, C_{1}$ не зависит от $M_{0}$ и $M$; далее будем обозначать $Q^{*}\left(M^{\prime}, M_{0}^{\prime}\right)=$ $Q\left(M, M_{0}\right)$.

Рассмотрим выражение

$$
\begin{aligned}
I & =\iint_{\mathbb{R}_{2}} J\left(M^{\prime}, M_{0}^{\prime}\right) q\left(x_{0}, y_{0}\right) d x_{0} d y_{0} \\
& =\iint_{\mathbb{R}_{2}} J_{1}\left(M^{\prime}, M_{0}^{\prime}\right) q\left(x_{0}, y_{0}\right) d x_{0} d y_{0}+\iint_{\mathbb{R}_{2}} Q^{*}\left(M^{\prime}, M_{0}^{\prime}\right) q\left(x_{0}, y_{0}\right) d x_{0} d y_{0} \\
& =I_{1}\left(M^{\prime}\right)+I_{2}\left(M^{\prime}\right) .
\end{aligned}
$$

Из (7.14) и (7.15) следует неравенство

$$
\left|I_{2}\left(M^{\prime}\right)\right| \leqslant C_{3},
$$

где $C_{3}$ не зависит от $M^{\prime}$.

Рассмотрим представление

$$
\begin{aligned}
I_{1}\left(M^{\prime}\right)= & \iint_{S\left(R, M^{\prime}\right)} J_{1}\left(M^{\prime}, M_{0}^{\prime}\right) q\left(x_{0}, y_{0}\right) d x_{0} d y_{0} \\
& +\iint_{\mathbb{R}_{2} \backslash S\left(R, M^{\prime}\right)} J_{1}\left(M^{\prime}, M_{0}^{\prime}\right) q\left(x_{0}, y_{0}\right) d x_{0} d y_{0} \\
= & I_{11}\left(M^{\prime}\right)+I_{12}\left(M^{\prime}\right),
\end{aligned}
$$

где $S\left(R, M^{\prime}\right)$ - круг радиуса $R$ с центром в точке $M^{\prime}$.

Для интеграла $I_{12}\left(M^{\prime}\right)$ имеет место оценка

$$
\left|I_{12}\left(M^{\prime}\right)\right| \leqslant \frac{C_{3}}{R} .
$$

В работе [6] для интеграла по Адамару $I_{11}\left(M^{\prime}\right)$ доказано представление

$$
\begin{aligned}
I_{11}\left(M^{\prime}\right)= & \lim _{\varepsilon \rightarrow 0}\left[\iint_{S\left(R, M^{\prime}\right) \backslash S\left(\varepsilon, M^{\prime}\right)} \frac{k_{1}(x, y, \varphi)}{r^{2}} d r d \varphi-\frac{M_{1}(x, y)}{\varepsilon}\right] \\
& +\lim _{\varepsilon \rightarrow 0} \iint_{S\left(R, M^{\prime}\right) \backslash S\left(\varepsilon, M^{\prime}\right)} \frac{k_{2}(x, y, \varphi)}{r^{2}} d r d \varphi \\
& +\iint_{S\left(R, M^{\prime}\right)} k_{3}(x, y, r, \varphi) d r d \varphi \\
= & I_{21}\left(M^{\prime}\right)+I_{31}\left(M^{\prime}\right)+I_{41}\left(M^{\prime}\right)
\end{aligned}
$$

где

$$
\begin{aligned}
\left|k_{i}(x, y, r, \varphi)\right|<C, \quad i=1,2,3 ; & M_{1}(x, y)=q(x, y) \int_{0}^{2 \pi} \frac{d \psi}{L^{3 / 2}(x, y, \psi)} \\
L(x, y, \psi)=1+\cos ^{2} \psi A^{2}(x, y) ; \quad A^{2}(x, y) & =\left(f_{x}^{\prime}\right)^{2}(x, y)+\left(f_{y}^{\prime}\right)^{2}(x, y) ; \\
\int_{0}^{2 \pi} k_{2}(x, y, \varphi) d \varphi & =0 .
\end{aligned}
$$


Отсюда следует, что $I_{31}\left(M^{\prime}\right)=0$; в той же работе [6] доказано, что $I_{21}\left(M^{\prime}\right)=$ $-M_{1}(x, y) / R$.

Из сделанных выше замечаний следует оценка

$$
\left|I_{11}\left(M^{\prime}\right)\right| \leqslant C\left(R+\frac{1}{R}\right) .
$$

Из неравенств (7.16), (7.18), (7.20) и из произвольности выбора $R$ вытекает

$$
\left|I\left(M^{\prime}\right)\right| \leqslant C_{0}
$$

где $C_{0}$ не зависит от $M^{\prime}$.

ЗАмЕчАнИЕ 7.1. Из неравенства (7.21) следует утверждение: для любой ограниченной области $D \subset \mathbb{R}_{2}$ существует ограниченная область $D_{1} \supset D$ такая, что для точек $M^{\prime} \in D$ будет выполняться неравенство

$$
\left|\iint_{D_{1}} J\left(M^{\prime}, M_{0}^{\prime}\right) q\left(x_{0}, y_{0}\right) d x_{0} d y_{0}\right| \leqslant K
$$

где константа $K$ не зависит от $M^{\prime}, D$ u $D_{1}$.

Введем семейство дискретных операторов $\mathrm{T}_{h}$ соотношением

$$
\pi \mathrm{T}_{h} u(x, h)=\sum_{k} u(k) \beta_{1}(k, m),
$$

г де $\beta_{1}(k, m)$ определяются равенствами (7.10), оператор $\pi$ см. в [2; определение 1.2], $u(k)=\pi u(x, h)$.

ПрЕДЛОЖЕНИЕ 7.3. Если $f(x, y) \in C_{0}^{4}\left(\mathbb{R}_{2}\right)$, то семейство операторов $\mathrm{T}_{h}$ ограниченно отображсает пространства $M(r, h)$ в пространства $M(r-1, h)$, $0<r<1$.

ДокАЗАТЕЛЬСтво. Ввиду замечания 5.1 из [2] утверждение достаточно доказать для функций $u(x, h) \in M_{h}^{\infty}, u(x, h) \in M(r, h), 0<r<1$. Рассмотрим линейный функционал $I=(2 \pi)^{-2} l(\nu(x, h))$ над $M(1-r, h)$, определенный элементом $\mathrm{T}_{h} u(x, h)$. Для доказательства предложения 7.3 достаточно установить неравенство

$$
|I(\nu(x, h))| \leqslant C\|\nu(x, h)\|_{M(1-r, h)} \cdot\|u(x, h)\|_{M(r, h)} \forall \nu(x, h) \in M(1-r, h) .
$$

В свою очередь, ввиду замечания 5.1 из [2] неравенство (7.24) достаточно доказать для функций $\nu(x, h) \in M_{h}^{\infty}, \nu(x, h) \in M(1-r, h)$. Итак, пусть $u(x, h)$, $\nu(x, h) \in M_{0}^{\infty}(h)$. Тогда согласно замечанию 7.1 сушествует ограниченная область $D_{1}, D_{1} \supset \operatorname{supp} u(x, h), D_{1} \supset \operatorname{supp} \nu(x, h)$, такая, что для любого разбиения на ячейки $D(m, h), D(m, h) \subset \operatorname{supp} u(x, h)$ либо $D(m, h) \subset \operatorname{supp} \nu(x, h)$, будет выполняться неравенство

$$
\left|\sum_{k \in \Lambda(h)} \beta_{1}(k, m)\right| \leqslant C_{0},
$$


где $\Lambda(h)$ - множество номеров $k$ таких, что ячейки $D(k, h) \subset D_{1} ; C_{0}$ - константа, не зависяшая от $m$ и $D_{1}$.

Далее имеем из [2; формула (6.5)]:

$$
\begin{aligned}
I(\nu(x, h))= & h^{2} \sum_{m \in \Lambda(h)} \nu(m) \sum_{k \in \Lambda(h)} \overline{u(k)} \beta_{1}(k, m) \\
= & h^{2} \sum_{m \in \Lambda(h)} \beta_{2}(m) \nu_{1}(m) \sum_{k \in \Lambda(h)} \overline{u(k)} \beta_{1}(k, m) \\
= & h^{2} \sum_{m \in \Lambda(h)} \beta_{2}(m) \nu_{1}(m)\left[\sum_{\substack{k \in \Lambda(h) \\
k \neq m}} \overline{u(k)} \beta_{1}(k, m)+\beta_{1}(m, m) \overline{u(m)}\right] \\
= & h^{2} \sum_{m \in \Lambda(h)} \beta_{2}(m) \nu_{1}(m)\left[\sum_{k \in \Lambda(h)} \beta_{1}(k, m) \cdot(\overline{u(k)}-\overline{u(m)})\right] \\
& +h^{2} \sum_{m \in \Lambda(h)} \beta_{2}(m) \beta(m) \nu_{1}(m) \overline{u(m)} \\
= & I_{1}(\nu(x, h))+I_{2}(\nu(x, h))
\end{aligned}
$$

где $\nu_{1}(m)=\nu(m) / \beta_{2}(m), \nu(m)=\pi \nu(x, h), \beta(m)=\sum_{k \in \Lambda(h)} \beta_{1}(k, m)$.

Легко проверить, что семейство ступенчатых функций $\psi(x, h)$ таких, что $\pi \psi(x, h)=1 / \beta_{2}(m)$, является липшицевым (см. определение 6.1). Из предложения 6.1 следует, что $\nu_{1}(x, h) \in M(1-r, h), \pi \nu_{1}(x, h)=\nu_{1}(m)$.

Из (7.25) и сделанњых замечаний следует неравенство

$$
\begin{aligned}
\left|I_{2}(\nu(x, h))\right| & \leqslant C_{0}\|\nu(x, h)\|_{L_{2}\left(\mathbb{R}_{n}\right)} \cdot\|u(x, h)\|_{L_{2}\left(\mathbb{R}_{n}\right)} \\
& \leqslant C_{1}\|M(x, h)\|_{M(1-r, h)} \cdot\|u(x, h)\|_{M(r, h)} .
\end{aligned}
$$

Поменяем местами индексы $k$ и $m$ в $I_{1}(\nu(x, h))$ :

$$
\begin{aligned}
I_{1}= & h^{2} \sum_{k \in \Lambda(h)} \beta_{2}(k) \nu_{1}(k)\left[\sum_{m \in \Lambda(h)} \beta_{1}(m, k)[\overline{u(m)}-\overline{u(k)}]\right] \\
= & h^{2} \sum_{k \in \Lambda(h)} \sum_{m \in \Lambda(h)} \beta_{2}(m) \beta_{1}(k, m) \nu_{1}(k)[\overline{u(m)}-\overline{u(k)}] \\
& +h^{2} \sum_{k \in \Lambda(h)} \sum_{m \in \Lambda(h)}\left[\beta_{2}(k) \beta_{1}(k, m)-\beta_{2}(m) \beta_{1}(k, m)\right] \cdot \nu_{1}(k)[\overline{u(m)}-\overline{u(k)}] .
\end{aligned}
$$

Сравнивая последнее соотношение с (7.26), получаем

$$
\begin{aligned}
I_{1}(\nu(x, h))= & \frac{h^{2}}{2} \sum_{k \in \Lambda(h)} \sum_{m \in \Lambda(h)} \beta_{2}(m) \beta_{1}(k, m)\left[\nu_{1}(k)-\nu_{1}(m)\right][\overline{u(m)}-\overline{u(k)}] \\
& +\frac{h^{2}}{2} \sum_{k \in \Lambda(h)} \sum_{m \in \Lambda(h)} \Delta \gamma(k, m) \nu_{1}(k)[\overline{u(m)}-\overline{u(k)}] \\
= & A_{1}(\nu(x, h))+A_{2}(\nu(x, h))
\end{aligned}
$$


Рассмотрим величины $\alpha_{r}(k, m)=\left(|k-m|^{2+2 r} \cdot|h|^{2 r}\right)^{-1}, k \neq m, 0<r<1$. Имеет место соотношение $\alpha_{1 / 2}(k, m)=\left(\alpha_{r}(k, m) \cdot \alpha_{1-r}(k, m)\right)^{1 / 2}$. Из [2; предложение 4.1] следует неравенство

$h^{2} \sum_{\substack{m \in \Lambda(h) \\ k \neq m}} \sum_{k \in \Lambda(h)} \alpha_{r}(k, m)|u(m)-u(k)|^{2} \leqslant C\|u(x, h)\|_{M_{1}(r, h)}^{2} \leqslant C_{1}\|u(x, h)\|_{M(r, h)}^{2}$.

Из определения величин $\beta_{2}(m), \beta_{1}(k, m)$ (см. (7.10)) и предложения 7.2 при $k \neq m$ следуют оценки

$$
\left|\beta_{2}(m) \beta_{1}(k, m)\right| \leqslant C_{1} \alpha_{1 / 2}(k, m), \quad|\Delta \gamma(k, m)| \leqslant C_{1} h \sqrt{\alpha_{r}(k, m) \alpha_{1-r}(k, m)},
$$

где $C_{1}$ не зависит от $h, k$ и $m$.

Из (7.28)-(7.30) вытекают неравенства

$$
\begin{aligned}
& \left|A_{1}(\nu(x, h))\right| \leqslant C_{2} \sqrt{h^{2} \sum_{\substack{m \in \Lambda(h) \\
k \neq m}} \sum_{k \in \Lambda(h)} \alpha_{1-r}(k, m)\left|\nu_{1}(k)-\nu_{1}(m)\right|^{2}} \\
& \times \sqrt{h^{2} \sum_{\substack{m \in \Lambda(h) \\
k \neq m}} \sum_{\substack{k \in \Lambda(h)\\
}} \alpha_{r}(k, m)|u(m)-u(k)|^{2}} \\
& \leqslant C_{3}\|\nu(x, h)\|_{M(1-r, h)} \cdot\|u(x, h)\|_{M(r, h)} ; \\
& \left|A_{2}(\nu(x, h))\right| \leqslant C_{2} \sqrt{h^{2} \sum_{\substack{m \in \Lambda(h) \\
k \neq m}} \sum_{k \in \Lambda(h)} h^{2} \alpha_{1-r}(k, m)\left|\nu_{1}(k)\right|^{2}} \\
& \times \sqrt{h^{2} \sum_{\substack{m \in \Lambda(h) \\
k \neq m}} \sum_{\substack{k \in \Lambda(h)\\
}} \alpha_{r}(k, m)|u(m)-u(k)|^{2}} \\
& \leqslant C_{3} \cdot\|u(x, h)\|_{M(r, h)} \\
& \times \sqrt{h^{2} \sum_{k \in \Lambda(h)} h^{2 r}|\nu(k)|^{2} \sum_{\substack{m \in \Lambda(h) \\
k \neq m}} h^{2-2 r} \alpha_{1-r}(k, m)} .
\end{aligned}
$$

Из $(7.32)$, учитывая неравенство $\sum_{k \neq m} h^{2-2 r} \alpha_{1-r}(k, m) \leqslant C_{0}$, где $C_{0}$ не зависит от $h$ и $k$, получаем оценку

$$
\left|A_{2}(\nu(x, h))\right| \leqslant C_{4}\|u(x, h)\|_{M(r, h)} \cdot h^{r}\|\nu(x, h)\|_{L_{2}\left(\mathbb{R}_{2}\right)} .
$$

Из (7.27), (7.31) и (7.33) получим неравенство (7.24). Отсюда вытекает предложение 7.3 .

ПРЕДЛОЖЕНИЕ 7.4. Ограниченное семейство операторов $\mathrm{T}_{h}$ устойчиво отображает пространства $\stackrel{\circ}{M}\left(1 / 2, h, \Omega_{1 h}\right)$ в $M\left(-1 / 2, h, \Omega_{1 h}\right)$. 
ДоказАТЕЛЬСтво. Обозначим через $A(h)$ и $A_{1}(h)$ множества значений индек$\operatorname{coв} k=\left(k_{1}, k_{2}\right)$ таких, что соответственно выполняется $D(k, h) \subset \Omega, D(k, h) \subset \Phi$. Ввиду предположений, сделанных относительно $\sigma$, будут выполняться неравенства

$$
\begin{gathered}
\beta_{1}(m, m)<0 \quad \forall m \in A_{1}(h), \quad \sum_{k \in A(h)} \beta_{1}(k, m)<0, \\
\sum_{k \in A_{1}(h)} \beta_{1}(k, m)=\beta(m)<0 \quad \forall m \in A(h), \quad 0<C_{1} \leqslant|\beta(m)| \leqslant C_{2},
\end{gathered}
$$

где $C_{1}$ и $C_{2}$ не зависят от $h$ и $m$ для всякого $m \in A(h)$.

Рассмотрим линейньй функционал $I=(2 \pi)^{-2} l(\nu(x, h))$ над пространством $\stackrel{\circ}{M}\left(1 / 2, h, \Omega_{1 h}\right)$, определенный элементом $\mathrm{T}_{h} u(x, h), u(x, h) \in \stackrel{\circ}{M}\left(1 / 2, h, \Omega_{1 h}\right)$. Найдем его значения на семействе функций $\nu(x, h)=q(x, h) u(x, h)$, где $\pi q(x, h)=$ $\beta_{2}(k)$. Семейство функций $q(x, h)$ липшицево, поэтому $\nu(x, h) \in \stackrel{\circ}{M}\left(1 / 2, h, \Omega_{1 h}\right)$ по предложению 6.1.

Аналогично (7.26) и (7.28) получим соотношение

$$
\begin{aligned}
I(\nu(x, h))= & h^{2} \sum_{m \in A(h)} \beta_{2}(m) u(m) \sum_{k \in A(h)} \overline{u(k)} \beta_{1}(k, m) \\
= & h^{2} \sum_{m \in A_{1}(h)} \beta_{2}(m) u(m) \sum_{k \in A_{1}(h)} \overline{u(k)} \beta_{1}(k, m) \\
= & -\frac{h^{2}}{2} \sum_{k \in A_{1}(h)} \sum_{m \in A_{1}(h)} \beta_{2}(m) \beta_{1}(k, m)|u(m)-u(k)|^{2} \\
& +\frac{h^{2}}{2} \sum_{k \in A_{1}(h)} \sum_{m \in A_{1}(h)} \Delta \gamma(k, m) u(k)(\overline{u(m)}-\overline{u(k)}) \\
& -h^{2} \sum_{m \in A(h)} \beta_{2}(m)|\beta(m)||u(m)|^{2} .
\end{aligned}
$$

Из (7.4) и (7.35) получим

$$
\begin{aligned}
|I(\nu(x, h))| \geqslant & C_{1}\left[\frac{h^{2}}{2} \sum_{k \in A_{1}(h)} \sum_{m \in A_{1}(h)} \gamma^{*}(k, m)|u(m)-u(k)|^{2}\right. \\
& \left.+h^{2} \sum_{m \in A(h)} \beta_{2}(m)|\beta(m)||u(m)|^{2}\right] \\
& -\left|\frac{h^{2}}{2} \sum_{k \in A_{1}(h)} \sum_{m \in A_{1}(h)} \Delta \gamma(k, m) u(k)[\overline{u(m)}-\overline{u(k)}]\right| \\
= & I_{1}(\nu(x, h))-I_{2}(\nu(x, h)) .
\end{aligned}
$$

Рассмотрим семейство вихревых операторов $\mathrm{T}_{h, 1 / 2}$, определяемых соотношением

$$
\pi \mathrm{T}_{h, 1 / 2} u(x, h)=\sum_{k \in A(h)} u(k) \gamma^{*}(k, m)
$$

где $u(x, h) \in \stackrel{\circ}{M}\left(1 / 2, h, \Omega_{1 h}\right)$. 
Пусть $l_{1}=(2 \pi)^{-2} l(\nu(x, h))$ - линейный функционал над $\stackrel{\circ}{M}\left(1 / 2, h, \Omega_{1 h}\right)$, определяемый элементом $\mathrm{T}_{h, 1 / 2} u(x, h), \nu(x, h) \in \stackrel{\circ}{M}\left(1 / 2, h, \Omega_{1 h}\right)$. Рассмотрим его значения на семействе функций $u(x, h)$. Тогда аналогично $(7.35)$ получим:

$$
\begin{array}{r}
l_{1}(u(x, h))=-\frac{h^{2}}{2} \sum_{k \in A_{1}(h)} \sum_{m \in A_{1}(h)} \gamma^{*}(k, m)|u(m)-u(k)|^{2} \\
-h^{2} \sum_{m \in A(h)}\left|\beta^{*}(m)\right||u(m)|^{2},
\end{array}
$$

где $\Delta \gamma^{*}(k, m)=0 \quad \forall k, m ; \beta^{*}(m)=\sum_{k \in A_{1}(h)} \gamma^{*}(k, m)<0 ;$ при $m \in A(h)$ выполняется неравенство $0<C_{1}<\left|\beta^{*}(m)\right|<C_{2}$, константы $C_{1}$ и $C_{2}$ не зависят от $h$ и $m$.

Из предложения 5.1 следует неравенство

$$
\left|l_{1}(u(x, h))\right| \geqslant k \cdot\|u(x, h)\|_{\stackrel{\circ}{\circ\left(1 / 2, h, \Omega_{1 h}\right)}},
$$

где $k$ не зависит от $h$.

Из (7.34), (7.36) и (7.38) вытекает оценка

$$
\left|I_{1}(\nu(x, h))\right| \geqslant k_{1} \cdot\|u(x, h)\|_{\stackrel{\circ}{\circ}\left(1 / 2, h, \Omega_{1 h}\right)}^{2},
$$

где $k_{1}$ не зависит от $h$.

Из (7.33) получим

$$
\left|I_{2}(\nu(x, h))\right| \leqslant k_{2} h^{1 / 2} \cdot\|u(x, h)\|_{\stackrel{\circ}{\circ}\left(1 / 2, h, \Omega_{1 h}\right)}^{2},
$$

где $k_{2}$ не зависит от $h$.

Из (7.40) следует: сушествует такое $\delta>0$, что при $|h|<\delta$ будет выполняться неравенство $|I(\nu(x, h))| \geqslant k_{3} \cdot \|\left. u(x, h)\right|_{M\left(1 / 2, h, \Omega_{1 h}\right)} ^{2}$, где $k_{3}$ не зависит от $h$. Из последнего неравенства вытекает устойчивость семейства операторов $\mathrm{T}_{h}$. Предложение 7.4 доказано.

ПРЕДЛОЖЕНИЕ 7.5. Если $f(x, y) \in C_{0}^{4}\left(\mathbb{R}_{2}\right)$, mo оператор

$$
\mathrm{T}(u)=\iint_{\mathbb{R}_{2}} J\left(M^{\prime}, M_{0}^{\prime}\right) q\left(x_{0}, y_{0}\right) u\left(x_{0}, y_{0}\right) d x_{0} d y_{0}
$$

ограничен из $H_{1 / 2}\left(\mathbb{R}_{2}\right)$ в $H_{-1 / 2}\left(\mathbb{R}_{2}\right)$. 
ДокАЗАтЕльство. Рассмотрим линейный функционал $I=(2 \pi)^{-2} l(\nu(x, h))$ над пространством $H_{1 / 2}\left(\mathbb{R}_{2}\right)$, определенньй элементом $\mathrm{T}(u)$. Тогда для доказательства предложения 7.5 достаточно для всех $u(x, y), \nu(x, y) \in H_{1 / 2}\left(\mathbb{R}_{2}\right)$ установить неравенство

$$
|I(\nu)| \leqslant C\|u(x, y)\|_{H_{1 / 2}\left(\mathbb{R}_{2}\right)} \cdot\|\nu(x, y)\|_{H_{1 / 2}\left(\mathbb{R}_{2}\right)} .
$$

Так как пространство $C_{0}^{\infty}\left(\mathbb{R}_{2}\right)$ всюду плотно в $H_{1 / 2}\left(\mathbb{R}_{2}\right)$, то неравенство $(7.41)$ достаточно доказать для функций $u(x, y), \nu(x, y) \in C_{0}^{\infty}\left(\mathbb{R}_{2}\right)$. Кроме того, так как оператор $Q, Q \nu=q(x, y) \nu(x, y)$, взаимно однозначно и ограниченно отображает $H_{1 / 2}\left(\mathbb{R}_{2}\right)$ на $H_{1 / 2}\left(\mathbb{R}_{2}\right)$, то неравенство $(7.41)$ достаточно доказать для $I(Q \nu)$, где $q(x, y)=\sqrt{1+f_{x}^{\prime 2}+f_{y}^{\prime 2}}$.

Из замечания 7.1 следует: существует область $\Omega_{1} \supset \Omega$ такая, что будет выполняться неравенство

$$
\left|\iint_{\Omega_{1}} J\left(M^{\prime}, M_{0}^{\prime}\right) q\left(x_{0}, y_{0}\right) d x_{0} d y_{0}\right|<k
$$

для любой точки $M^{\prime} \in \Omega, k$ не зависит от $M^{\prime}$ и областей $\Omega_{1}, \Omega(\Omega \supset \operatorname{supp} u(x, y)$, $\Omega \supset \operatorname{supp} \nu(x, y))$.

Далее рассмотрим

$$
\begin{aligned}
I(Q \nu)= & \iint_{\Omega} q(x, y) \nu(x, y)\left[\iint_{\Omega} J\left(M^{\prime}, M_{0}^{\prime}\right) \overline{u\left(x_{0}, y_{0}\right)} q(x, y) d x_{0} d y_{0}\right] d x d y \\
= & \iint_{\Omega_{1}} q(x, y) \nu(x, y)\left[\iint_{\Omega_{1}} J\left(M^{\prime}, M_{0}^{\prime}\right) \overline{u\left(x_{0}, y_{0}\right)} q(x, y) d x_{0} d y_{0}\right] d x d y \\
= & \iint_{\Omega_{1}} q(x, y) \nu(x, y)\left[\iint_{\Omega_{1}} J\left(M^{\prime}, M_{0}^{\prime}\right) \overline{u\left(x_{0}, y_{0}\right)} q(x, y) d x_{0} d y_{0}\right. \\
& \left.-\overline{u(x, y)} \iint_{\Omega_{1}} J\left(M^{\prime}, M_{0}^{\prime}\right) q\left(x_{0}, y_{0}\right) d x_{0} d y_{0}+\overline{u(x, y)} \cdot \beta(x, y)\right] d x d y \\
= & \iint_{\Omega_{1}} q(x, y) \nu(x, y) \\
& \times\left[\iint_{\Omega_{1}} J\left(M^{\prime}, M_{0}^{\prime}\right)\left[\overline{u\left(x_{0}, y_{0}\right)}-\overline{u(x, y)}\right] q\left(x_{0}, y_{0}\right) d x_{0} d y_{0}\right] d x d y \\
& +\iint_{\Omega} q(x, y) \beta(x, y) \nu(x, y) \overline{u(x, y)} d x d y
\end{aligned}
$$

где

$$
\beta(x, y)=\int_{\Omega_{1}} J\left(M^{\prime}, M_{0}^{\prime}\right) q\left(x_{0}, y_{0}\right) d x_{0} d y_{0}
$$

и $|\beta(x, y)|<k$ при $(x, y) \in \Omega($ см. (7.42)). 
Меняя местами в (7.43) $x$ и $x_{0}, y$ и $y_{0}$, а также пользуясь симметричностью $J\left(M^{\prime}, M_{0}^{\prime}\right)$, получаем

$$
\begin{aligned}
I(Q \nu)= & \int_{\Omega_{1}}\left[\int_{\Omega_{1}} J\left(M^{\prime}, M_{0}^{\prime}\right) q(x, y) q\left(x_{0}, y_{0}\right) \nu\left(x_{0}, y_{0}\right)\right. \\
& \left.\times\left[\overline{u(x, y)}-\overline{u\left(x_{0}, y_{0}\right)}\right] d x d y\right] d x_{0} d y_{0} \\
& +\iint_{\Omega} q(x, y) \beta(x, y) \nu(x, y) \cdot \overline{u(x, y)} d x d y
\end{aligned}
$$

Из (7.43) и (7.44) следует соотношение

$$
\begin{aligned}
& I(Q \nu)=-\frac{1}{2} \int_{\Omega_{1}} \int_{\Omega_{1}} J\left(M^{\prime}, M_{0}^{\prime}\right) q(x, y) q\left(x_{0}, y_{0}\right) \\
& \quad \times\left[\nu(x, y)-\nu\left(x_{0}, y_{0}\right)\right]\left[\overline{u(x, y)}-\overline{u\left(x_{0}, y_{0}\right)}\right] d x_{0} d y_{0} d x d y \\
& \quad+\iint_{\Omega} q(x, y) \beta(x, y) \nu(x, y) \overline{u(x, y)} d x d y=I_{1}(Q \nu)+I_{2}(Q \nu) .
\end{aligned}
$$

Из (7.42) вытекает неравенство

$$
\left|I_{2}(Q \nu)\right| \leqslant C\|u(x, y)\|_{L_{2}(\Omega)} \cdot\|\nu(x, y)\|_{L_{2}(\Omega)} \cdot
$$

Так как интегралы в $I_{1}(Q \nu)$ абсолютно сходятся, то, применяя $(7.4)$ и неравенство Коши-Буняковского, получаем неравенство

$$
\begin{aligned}
\left|I_{1}(Q \nu)\right| \leqslant & C_{1} \sqrt{\iint_{\Omega_{1}} \iint_{\Omega_{1}} \frac{\left|\nu(x, y)-\nu\left(x_{0}, y_{0}\right)\right|^{2} d x d y d x_{0} d y_{0}}{\left[\left(x-x_{0}\right)^{2}+\left(y-y_{0}\right)^{2}\right]^{3 / 2}}} \\
& \times \sqrt{\iint_{\Omega_{1}} \iint_{\Omega_{1}} \frac{\left|u(x, y)-u\left(x_{0}, y_{0}\right)\right|^{2} d x d y d x_{0} d y_{0}}{\left[\left(x-x_{0}\right)^{2}+\left(y-y_{0}\right)^{2}\right]^{3 / 2}}} \\
\leqslant & C_{2}\|\nu(x, y)\|_{\stackrel{\circ}{H}_{1 / 2}(\Omega)} \cdot\|u(x, y)\|_{\stackrel{\circ}{H}_{1 / 2}(\Omega)} \cdot
\end{aligned}
$$

Из неравенств (7.46) и (7.47) следует (7.41). Итак, предложение 7.5 доказано.

Исследуем свойства функции

$$
\nu(x, y)=\iint_{\Omega} J\left(M^{\prime}, M_{0}^{\prime}\right) u\left(x_{0}, y_{0}\right) d x_{0} d y_{0},
$$

где $u\left(x_{0}, y_{0}\right) \in C_{0}^{3}(\Omega)$.

Функцию $\nu(x, y)$ представим в виде $\nu(x, y)=\nu_{1}(x, y)+\nu_{2}(x, y)$, где

$$
\begin{aligned}
& \nu_{1}(x, y)=\iint_{\Omega} J_{1}\left(M^{\prime}, M_{0}^{\prime}\right) u\left(x_{0}, y_{0}\right) d x_{0} d y_{0} \\
& \nu_{2}(x, y)=\iint_{\Omega} Q^{*}\left(M^{\prime}, M_{0}^{\prime}\right) u\left(x_{0}, y_{0}\right) d x_{0} d y_{0}
\end{aligned}
$$

а функция $Q^{*}\left(M^{\prime}, M_{0}^{\prime}\right)$ определена формулой $(7.14)$. 
Так как $f(x, y) \in C_{0}^{4}\left(\mathbb{R}_{2}\right)$, то функция

$$
\alpha(x, y, r, \varphi)=\frac{f(x, y)-f(x+r \cos \varphi, y+r \sin \varphi)}{r}
$$

имеет производные $\frac{\partial \alpha}{\partial r}=C^{1}\left(\mathbb{R}_{2} \times \mathbb{R}_{2}\right), \frac{\partial^{2} \alpha}{\partial r^{2}}=C\left(\mathbb{R}_{2} \times \mathbb{R}_{2}\right)$. Поэтому если ввести переменные $r=\sqrt{\left(x-x_{0}\right)^{2}+\left(y-y_{0}\right)^{2}}, x-x_{0}=-r \cos \varphi, y-y_{0}=-r \sin \varphi$, то функция $F(x, y, r, \varphi)=1 /\left[1+\alpha^{2}\right]^{3 / 2}$ имеет непрерьвные по всем переменным $x, y$, $r, \varphi$ производные $\frac{\partial F}{\partial r}$ и $\frac{\partial^{2} F}{\partial r^{2}}$. Отсюда следует, что функцию $J_{1}\left(M^{\prime}, M_{0}^{\prime}\right)$ можно

$$
J_{1}\left(M^{\prime}, M_{0}^{\prime}\right)=\frac{1}{r^{3}} A_{0}(x, y, \varphi)+\frac{1}{r^{2}} A_{1}(x, y, \varphi)+\frac{A_{2}(x, y, r, \varphi)}{r},
$$

где $A_{0}(x, y, \varphi)=F(x, y, \varphi, 0), A_{1}(x, y, \varphi)=\left.\frac{\partial F}{\partial r}\right|_{r=0}, A_{2}(x, y, r, \varphi)=\frac{O(x, y, r, \varphi)}{r^{2}}$, $O(x, y, r, \varphi)$ - остаточный член формулы Тейлора, $A_{2}(x, y, r, \varphi)$ непрерывна по переменньм $x, y, r, \varphi$.

В работе [6] показано, что имеет место

$$
\begin{gathered}
\int_{0}^{2 \pi} A_{1}(x, y, \varphi) d \varphi=0 \\
\int_{0}^{2 \pi} A_{0}(x, y, \varphi) \cos \varphi d \varphi=0, \quad \int_{0}^{2 \pi} A_{0}(x, y, \varphi) \sin \varphi d \varphi=0 .
\end{gathered}
$$

Функция $A_{0}(x, y, \varphi)$ имеет непрерывные производные по всем аргументам до 3-го порядка, функция $A_{1}(x, y, \varphi)$ имеет непрерывные производные по всем аргументам до второго порядка включительно.

Возьмем число $R>0$ настолько большим, чтобы круг $S\left(R, M^{\prime}\right)$ радиуса $R$ с центром в любой точке $M^{\prime}(x, y)$ полностью содержал $\Omega$. Тогда функцию $\nu_{1}(x, y)$ можно представить в виде

$$
\begin{aligned}
\nu_{1}(x, y)= & \lim _{\varepsilon \rightarrow 0}\left[\iint_{S\left(R, M^{\prime}\right) \backslash S\left(\varepsilon, M^{\prime}\right)} \frac{u(x+r \cos \varphi, y+r \sin \varphi)}{r^{2}} \cdot A_{0}(x, y, \varphi) d r d \varphi\right. \\
& \left.-\frac{u(x, y) B(x, y)}{\varepsilon}\right] \\
& +\lim _{\varepsilon \rightarrow 0}\left[\iint_{S\left(R, M^{\prime}\right) \backslash S\left(\varepsilon, M^{\prime}\right)} \frac{u(x+r \cos \varphi, y+r \sin \varphi)}{r} A_{1}(x, y, \varphi) d r d \varphi\right. \\
& \left.+\iint_{S\left(R, M^{\prime}\right) \backslash S\left(\varepsilon, M^{\prime}\right)} u(x+r \cos \varphi, y+r \sin \varphi) A_{2}(x, y, r, \varphi) d r d \varphi\right] \\
= & \lambda_{1}(x, y)+\lambda_{2}(x, y)+\lambda_{3}(x, y),
\end{aligned}
$$

где

$$
\begin{gathered}
B(x, y)=\int_{0}^{2 \pi} \frac{d \psi}{L^{3 / 2}(x, y, \psi)} \\
L(x, y, \psi)=1+\cos ^{2} \psi A^{2}(x, y), \quad A^{2}(x, y)=f_{x}^{\prime 2}(x, y)+f_{y}^{\prime 2}(x, y) .
\end{gathered}
$$


Из теоремы о непрерывности интеграла по параметру и свойств $A_{2}(x, y, r, \varphi)$ и $u\left(x_{0}, y_{0}\right)$ следует, что $\lambda_{3}(x, y)$ непрерывна по аргументам $x$ и $y$.

Используя (7.50), а также тот факт, что $u(x+R \cos \varphi, y+R \sin \varphi)=0$, получаем, интегрируя по частям,

$$
\begin{aligned}
\lambda_{2}(x, y)=\int_{0}^{2 \pi} \int_{0}^{R} A_{1}(x, y, \varphi)\left[\frac{\partial u(x+r \cos \varphi, y+r \sin \varphi)}{\partial x} \cos \varphi\right. & \\
& \left.+\frac{\partial u(x+r \cos \varphi, y+r \sin \varphi)}{\partial y} \sin \varphi\right] \ln r d r d \varphi
\end{aligned}
$$

Отсюда аналогично выводу относительно функции $\lambda_{3}(x, y)$ заключаем, что функция $\lambda_{2}(x, y)$ непрерывна по аргументам $x$ и $y$.

Интегрируя по частям и используя соотношения (7.49) и (7.50), представляем функцию $\lambda_{1}(x, y)$ в виде

$$
\begin{aligned}
& \lambda_{1}(x, y)=-\int_{0}^{2 \pi} \int_{0}^{R} A_{0}(x, y, \varphi)\left[\frac{\partial^{2} u(x+r \cos \varphi, y+r \sin \varphi)}{\partial x^{2}} \cos ^{2} \varphi\right. \\
& \left.+\sin 2 \varphi \frac{\partial^{2} u(x+r \cos \varphi, y+r \sin \varphi)}{\partial x \partial y}+\sin ^{2} \varphi \frac{\partial^{2} u(x+r \cos \varphi, y+r \sin \varphi)}{\partial y^{2}}\right] \ln r d r d \varphi .
\end{aligned}
$$

Отсюда аналогично вьводу относительно $\lambda_{3}(x, y)$ и $\lambda_{2}(x, y)$ заключаем, что функция $\lambda_{1}(x, y) \in C\left(\mathbb{R}_{2}\right)$. Итак, $\nu_{1}(x, y) \in C\left(\mathbb{R}_{2}\right)$.

Функцию $\nu_{2}(x, y)$ представим в виде

$$
\begin{aligned}
\nu_{2}(x, y)= & \iint_{\Omega} \frac{\left(\mathbf{n}_{M_{0}}, \mathbf{n}_{M}\right)-1}{\left|M_{0} M\right|^{3}} u\left(x_{0}, y_{0}\right) d x_{0} d y_{0} \\
& +\iint_{\Omega} \frac{(-3)\left(\mathbf{M}_{0} \mathbf{M}, \mathbf{n}_{M}\right)\left(\mathbf{M}_{0} \mathbf{M}, \mathbf{n}_{M_{0}}\right)}{\left|M_{0} M\right|^{3}} u\left(x_{0}, y_{0}\right) d x_{0} d y_{0} \\
= & \beta_{1}(x, y)+\beta_{2}(x, y) .
\end{aligned}
$$

Переходя к координатной форме записи и вводя координаты

$$
r=\sqrt{\left(x-x_{0}\right)^{2}+\left(y-y_{0}\right)^{2}}, \quad x_{0}-x=r \cos \varphi, \quad y_{0}-y=r \sin \varphi,
$$

получаем соотношения

$$
\begin{gathered}
\frac{\left(\mathbf{M}_{0} \mathbf{M}, \mathbf{n}_{M_{0}}\right)}{r^{2}}=\gamma_{1}(x, y, r, \varphi), \quad \frac{\left(\mathbf{M}_{0} \mathbf{M}, \mathbf{n}_{M}\right)}{r^{2}}=\gamma_{2}(x, y, r, \varphi), \\
\frac{\left(\mathbf{n}_{M_{0}}, \mathbf{n}_{M}\right)}{r^{2}}=\frac{1}{r^{2}}+\gamma_{3}(x, y, r, \varphi) \\
\frac{1}{\left|M_{0} M\right|^{3}}=\frac{1}{r^{3}} F_{1}(x, y, r, \varphi), \quad \frac{1}{\left|M_{0} M\right|^{5}}=\frac{1}{r^{5}} F_{2}(x, y, r, \varphi),
\end{gathered}
$$

где функции $\gamma_{i}(x, y, r, \varphi), i=1,2,3, F_{j}(x, y, r, \varphi), j=1,2$, непрерывны по аргументам $x, y, r, \varphi$.

Из (7.51) и (7.52) вытекает, что $\beta_{1}(x, y), \beta_{2}(x, y) \in C\left(\mathbb{R}_{2}\right)$, следовательно, $\nu_{2}(x, y) \in C\left(\mathbb{R}_{2}\right)$.

Предыдущие рассуждения доказывают 
ПрЕДЛОЖЕНИЕ 7.6. Если $f(x, y) \in C_{0}^{4}\left(\mathbb{R}_{2}\right), u(x, y) \in C_{0}^{3}(\Omega)$, то функиия

$$
\nu(x, y)=\iint_{\Omega} J\left(M^{\prime}, M_{0}^{\prime}\right) u\left(x_{0}, y_{0}\right) q\left(x_{0}, y_{0}\right) d x_{0} d y_{0}
$$

непрерывна по аргументам $x$ и $y$.

Докажем, что семейство дискретных операторов $\mathrm{T}_{h}$, определенных соотношением (7.23), $\prod_{h}^{*}$-аппроксимирует в области $\Omega$ ограниченный оператор $\mathrm{T}: \stackrel{\circ}{H}_{1 / 2}(\Omega) \rightarrow H_{-1 / 2}(\Omega)$,

$$
\mathrm{T}(u)=\iint_{\Omega} J\left(M^{\prime}, M_{0}^{\prime}\right) u\left(x_{0}, y_{0}\right) q\left(x_{0}, y_{0}\right) d x_{0} d y_{0} .
$$

Пусть $u\left(x_{0}, y_{0}\right) \in C_{0}^{3}(\Omega)$; приближенное значение $S\left(M_{0 m}^{\prime}\right)$ интеграла $\mathrm{T}(u)$ будем вычислять по формуле

$$
S\left(M_{0 m}^{\prime}\right)=\sum_{k \in \Lambda(h)} u\left(k_{1} h, k_{2} h\right) \cdot \beta_{1}(k, m),
$$

где $\Lambda(h)$ - множество номеров $k=\left(k_{1}, k_{2}\right)$ таких, что $D(k, h) \subset \Omega_{1 h}, M_{0 m}^{\prime}=$ $M\left(m_{1} h, m_{2} h\right), M_{0 m}^{\prime} \in D(m, h) \subset \Omega_{1 h}$.

Из результатов работы [6] следует:

$$
\left|S\left(M_{0 m}^{\prime}\right)-\nu\left(M_{0 m}^{\prime}\right)\right|=O(h),
$$

где $O(h) \rightarrow 0$ при $|h| \rightarrow 0$ равномерно для всех $M_{0 m}^{\prime} \in \Omega_{1 h}$, а

$$
\nu\left(M_{0 m}^{\prime}\right)=\iint_{\Omega} J\left(M_{0 m}^{\prime}, M_{0}^{\prime}\right) u\left(x_{0}, y_{0}\right) q\left(x_{0}, y_{0}\right) d x_{0} d y_{0} .
$$

Из (7.53) и предложения 7.6 вытекает, что

$$
\int_{\Omega_{1 h}}|S(x, h)-\nu(x, y)|^{2} d x d y=O_{1}(h)
$$

где $O_{1}(h) \rightarrow 0$ при $h \rightarrow 0, h>0, \pi S(x, h)=S\left(M_{0 m}^{\prime}\right)$ (оператор $\pi$ задается в [2; определение 1.2]).

Итак, семейство операторов $\mathrm{T}_{h} \prod_{h}^{*}$-аппроксимирует в области $\Omega$ оператор $\mathrm{T}$ (см. определение 1.7).

Рассмотрим уравнение

$$
\mathrm{PT}(u)=g(x, y),
$$

где $u(x, y) \in \stackrel{\circ}{H}_{1 / 2}(\Omega), g(x, y) \in H_{-1 / 2}(\Omega), \mathrm{P}$ - оператор сужения на область $\Omega$.

Для нахождения приближенного решения $u(x, y, h)$ уравнения $(7.54)$ рассмотрим систему уравнений

$$
\mathrm{P}_{h} \mathrm{~T}_{h} u(x, y, h)=g(x, y, h),
$$

где $\mathrm{P}_{h}$ - сужение оператора $\mathrm{T}_{h}$ на область $\Omega_{1 h}, u(x, y, h) \in \stackrel{\circ}{M}\left(1 / 2, h, \Omega_{1 h}\right)$, $g(x, y, h) \in M\left(-1 / 2, h, \Omega_{1 h}\right)$, причем сушествуют продолжения $\widetilde{g}(x, y, h) \in$ $M(-1 / 2, h)$ функций $g(x, y, h)$ такие, что $\widetilde{g}(x, y, h) \rightarrow \lg (x, y)$ по норме $H_{-1 / 2}\left(\mathbb{R}_{2}\right)$ при $h \rightarrow 0$, где $\lg (x, y)$ - некоторое продолжение функции $g(x, y), \lg (x, y) \in$ $H_{-1 / 2}\left(\mathbb{R}_{2}\right)$. 
ТЕОРема 7.1. Пусть $\sigma$ - поверхность, описанная в начале $§ 7$. Тогда решение $u(x, y) \in \stackrel{\circ}{H}_{1 / 2}(\Omega)$ уравнения (7.54) существует и единственно для любой $g(x, y) \in H_{-1 / 2}(\Omega)$. Кроме того, найдется такое $\delta>0$, что при $0<h<\delta$ решения $u(x, y, h)$ системы (7.55) будут существовать, причем функиии и $(x, y, h)$ сходятся к точному решению $и(x, y)$ в $\stackrel{\circ}{H}_{r}(\Omega)$ для любого $r$, $0<r<\frac{1}{2}$, и имеет место соотношение

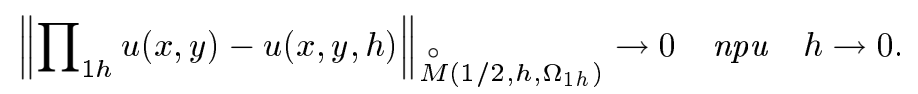

Доказательство теоремы 7.1 следует из предложений 7.3-7.6, а также из предложения 2.3 .

\section{§8. Дифракция на поверхности}

Рассмотрим уравнение Гельмгольца

$$
\begin{gathered}
\Delta u+\lambda^{2} u=0, \quad x \in \mathbb{R}_{3} \backslash \sigma, \\
\left.\frac{\partial u}{\partial n}\right|_{\sigma}=g(M),
\end{gathered}
$$

где $\sigma$ - поверхность, удовлетворяющая условиям $\S 7$.

Пусть относительно функции $u$ вьполняются условия излучения Зоммерфельда:

$$
u(x)=O\left(|x|^{-1}\right), \quad \frac{\partial u(x)}{\partial|x|}+i \lambda u(x)=o\left(|x|^{-1}\right) \text { при }|x| \rightarrow \infty,
$$

число $\lambda$ удовлетворяет условиям $\operatorname{Re} \lambda>0, \operatorname{Im} \lambda \leqslant 0$.

Если решать задачу (8.1) с условиями (8.2) через потенциал двойного слоя, то относительно функции $\nu\left(M_{0}\right)$, плотности потенциала двойного слоя, возникает уравнение

$$
\begin{aligned}
\int_{\sigma_{M_{0}}} G\left(M, M_{0}\right) \nu\left(M_{0}\right) d \sigma_{M_{0}}+ & \int_{\sigma_{M_{0}}} K_{1}\left(M, M_{0}\right) \nu\left(M_{0}\right) d \sigma_{M_{0}} \\
& +\int_{\sigma_{M_{0}}} K_{2}\left(M, M_{0}\right) \nu\left(M_{0}\right) d \sigma_{M_{0}}=g\left(M_{0}\right),
\end{aligned}
$$

где функция $G\left(M, M_{0}\right)$ совпадает с ядром интеграла (7.1),

$$
\begin{aligned}
& K_{1}\left(M_{0}, M\right)=\frac{G_{1}\left(M_{0}, M\right)\left(\mathbf{M}_{0} \mathbf{M}, \mathbf{n}_{M_{0}}\right)\left(\mathbf{M}_{0} \mathbf{M}, \mathbf{n}_{M}\right)}{\left|M_{0} M\right|^{5}} \\
& K_{2}\left(M_{0}, M\right)=-\frac{G\left(M_{0}, M\right)\left(\mathbf{n}_{M_{0}}, \mathbf{n}_{M}\right)}{\left|M_{0} M\right|^{3}} \\
& G_{1}\left(M_{0}, M\right)=e^{-i \lambda\left|M_{0} M\right|}\left[-\lambda^{2}\left|M_{0} M\right|^{2}+3 i \lambda\left|M_{0} M\right|+3\right]-3, \\
& G_{2}\left(M_{0}, M\right)=e^{-i \lambda\left|M_{0} M\right|}+i \lambda e^{-i \lambda\left|M_{0} M\right|}-1 .
\end{aligned}
$$


Переходя к декартовым координатам так же, как в $\S 7$, и оставляя для ядер интегралов те же обозначения, получаем уравнение относительно функции $\nu(x, y)$, плотности потенциала двойного слоя:

$$
\begin{aligned}
& \iint_{\Omega} G\left(M^{\prime}, M_{0}^{\prime}\right) \nu\left(x_{0}, y_{0}\right) q\left(x_{0}, y_{0}\right) d x_{0} d y_{0} \\
& \quad+\iint_{\Omega} K_{1}\left(M^{\prime}, M_{0}^{\prime}\right) \nu\left(x_{0}, y_{0}\right) q\left(x_{0}, y_{0}\right) d x_{0} d y_{0} \\
& \quad+\iint_{\Omega} K_{2}\left(M^{\prime}, M_{0}^{\prime}\right) \nu\left(x_{0}, y_{0}\right) q\left(x_{0}, y_{0}\right) d x_{0} d y_{0}=F(x, y),
\end{aligned}
$$

где $q(x, y)=\sqrt{1+f_{x}^{\prime 2}(x, y)+f_{y}^{\prime 2}(x, y)}, M^{\prime}=M^{\prime}(x, y), M_{0}^{\prime}=M_{0}^{\prime}\left(x_{0}, y_{0}\right)$.

Аналогично предложению 7.6 доказывается

ПРЕДЛОЖЕНИЕ 8.1. Если Әля поверхности $\sigma$ выполнены условия $\S 7$, $\nu\left(x_{0}, y_{0}\right) \in C_{0}^{3}(\Omega)$, то функция $\psi(x, y) \in C\left(\mathbb{R}_{2}\right)$, әде

$$
\begin{gathered}
\psi(x, y)=\iint_{\Omega} G\left(M^{\prime}, M_{0}^{\prime}\right) \nu\left(x_{0}, y_{0}\right) q\left(x_{0}, y_{0}\right) d x_{0} d y_{0} \\
+\iint_{\Omega} P\left(M^{\prime}, M_{0}^{\prime}\right) \nu\left(x_{0}, y_{0}\right) q\left(x_{0}, y_{0}\right) d x_{0} d y_{0}=\psi_{1}(x, y)+\psi_{2}(x, y) \\
P\left(M^{\prime}, M_{0}^{\prime}\right)=K_{1}\left(M^{\prime}, M_{0}^{\prime}\right)+K_{2}\left(M^{\prime}, M_{0}^{\prime}\right)
\end{gathered}
$$

Разобьем плоскость $X O Y$ с векторным шагом $h=(h, h)$ на элементарные ячейки $D(k, h)$ (см. [2; определение 1.1]). Определим семейство дискретных операторов $\mathrm{B}_{h}$ соотношением

$$
\begin{aligned}
\pi \mathrm{B}_{h} u(x, h) & =\sum_{k \in \Lambda(h)} u(k) \beta_{1}(k, m)+\sum_{k \in \Lambda(h)} u(k) \beta_{2}(k, m) \\
& =\pi \mathrm{T}_{h} u(x, h)+\pi \mathrm{P}_{h} u(x, h)
\end{aligned}
$$

где $\Lambda(h)$ - множество номеров $k=\left(k_{1}, k_{2}\right)$ таких, что $D(k, h) \in \Omega_{1 h}$; оператор $\mathrm{T}_{h}$ - тот же самый, что и в $\S 7$, величины $\beta_{2}(k, m)$ определяются равенством

$$
\begin{gathered}
\beta_{2}(k, m)=\iint_{D(k, h)} P\left(M_{0 m}^{\prime}, M_{0}^{\prime}\right) q\left(x_{0}, y_{0}\right) d x_{0} d y_{0}, \\
M_{0 m}^{\prime}=M\left(m_{1} h_{1}, m_{2} h_{2}\right) \in D(m, h) \subset \Omega_{1 h}, \\
P\left(M^{\prime}, M_{0}^{\prime}\right)=K_{1}\left(M^{\prime}, M_{0}^{\prime}\right)+K_{2}\left(M^{\prime}, M_{0}^{\prime}\right), \quad \pi u(x, h)=u(k),
\end{gathered}
$$

оператор $\pi$ задается в [2; определение 1.2].

Для ядра $P\left(M^{\prime}, M_{0}^{\prime}\right)$ имеет место оценка

$$
\left|P\left(M^{\prime}, M_{0}^{\prime}\right)\right| \leqslant \frac{C}{\left|M^{\prime} M_{0}^{\prime}\right|}
$$


Из (8.9) следует неравенство

$$
\sum_{k \in \Lambda(h)}\left|\beta_{2}(k, m)\right| \leqslant C
$$

где $C$ не зависит от $h$ и $m$ таких, что $D(m, h) \in \Omega_{1 h}$.

Для приближенного вычисления значений функции $\psi(x, y)($ см. $(8.6))$ применим квадратурную формулу

$$
\begin{aligned}
S\left(M_{0 m}^{\prime}\right) & =S_{1}\left(M_{0 m}^{\prime}\right)+S_{2}\left(M_{0 m}^{\prime}\right) \\
& =\sum_{k \in \Lambda(h)} \nu\left(k_{1} h, k_{2} h\right) \beta_{1}(k, m)+\sum_{k \in \Lambda(h)} \nu\left(k_{1} h, k_{2} h\right) \beta_{2}(k, m),
\end{aligned}
$$

где $\nu(x, y) \in C_{0}^{3}(\Omega)$.

Для $S_{1}\left(M_{0 m}^{\prime}\right)$ в силу (7.53) имеет место $\left|S_{1}\left(M_{0 m}^{\prime}\right)-\psi_{1}\left(M_{0 m}^{\prime}\right)\right|=O_{1}(h)$, $O_{1}(h) \rightarrow 0$ при $h \rightarrow 0$ равномерно для всех $m$ таких, что $D(m, h) \subset \Omega_{1 h}$. Из (8.10) следует $\left|S_{2}\left(M_{0 m}^{\prime}\right)-\psi_{2}\left(M_{0 m}^{\prime}\right)\right|=O_{2}(h), O_{2}(h) \rightarrow 0$ при $h \rightarrow 0$ равномерно для всех $m$ таких, что $D(m, h) \subset \Omega_{1 h}$. Отсюда и из предложения 8.1 получаем, что $\|S(x, y, h)-\psi(x, y)\|_{L_{2}\left(\Omega_{1 h}\right)} \rightarrow 0$ при $h \rightarrow 0$.

Из предыдуших рассуждений вытекает

ПРЕДЛОЖЕНИЕ 8.2. Семейство операторов $\mathrm{B}_{h} \prod_{h}^{*}$-аппроксимирует в области $\Omega$ оператор $\mathrm{B}$, где $\mathrm{B}$ определяется левой частью (8.5).

Рассмотрим функцию $\theta(x) \in C^{\infty}[0, \infty), \theta(x) \geqslant 0, \theta(x)=1$ при $0 \leqslant x \leqslant R$, где $R$ таково, что внутренность круга $S\left(R, M^{\prime}\right)$ с центром в точке $M^{\prime} \in \bar{\Omega}$ радиуса $R$ содержит $\bar{\Omega} ; \theta(x)=0$ при $x>R_{0}$, где $R_{0}>R$. Положим $P_{1}\left(M^{\prime}, M_{0}^{\prime}\right)=$ $P\left(M^{\prime}, M_{0}^{\prime}\right) \cdot \theta\left(\left|M^{\prime} M_{0}^{\prime}\right|\right)$. Функция $P_{1}\left(M^{\prime}, M_{0}^{\prime}\right)=P\left(M^{\prime}, M_{0}^{\prime}\right)$ при $M^{\prime}, M_{0}^{\prime} \in \Omega$. Отсюда следует, что уравнение

$$
\begin{aligned}
\mathrm{B}_{1}(\nu)=\iint_{\Omega} G & \left(M^{\prime}, M_{0}^{\prime}\right) \nu\left(x_{0}, y_{0}\right) q\left(x_{0}, y_{0}\right) d x_{0} d y_{0} \\
& +\iint_{\Omega} P_{1}\left(M^{\prime}, M_{0}^{\prime}\right) \nu\left(x_{0}, y_{0}\right) q\left(x_{0}, y_{0}\right) d x_{0} d y_{0}=F(x, y),
\end{aligned}
$$

где $(x, y) \in \Omega$, эквивалентно уравнению (8.5). Обозначим первый член в $(8.12)$ через $\mathrm{T}(\nu)$, второй - через $\mathrm{P}_{1}(\nu)$.

ЗАмЕЧАНИЕ 8.1. ВВиду конструкции функции $P_{1}\left(M^{\prime}, M_{0}^{\prime}\right)$ предложение 8.1 остается справедливым и для оператора $\mathrm{B}_{1}(\nu)$.

ПРЕДЛОЖЕНИЕ 8.3. Оператор $\mathrm{B}_{1}(\nu)$ ограничен из $\stackrel{\circ}{H}_{1 / 2}(\Omega)$ в $H_{-1 / 2}\left(\mathbb{R}_{2}\right)$. 
ДокАЗАТЕЛЬСТво. В силу предложения 7.5 утверждение верно для оператора $\mathrm{T}(\nu)$. Поэтому предложение 8.3 достаточно доказать для оператора $\mathrm{P}_{1}(\nu)$. Обозначим $\psi_{2}(x, y)=\mathrm{P}_{1}(\nu)$.

Далее, используя неравенство Иенсена [7], получаем

$$
\begin{aligned}
& \iint_{\mathbb{R}_{2}}\left|\psi_{2}(x, y)\right|^{2} d x d y \\
& =\iint_{\mathbb{R}_{2}}\left|\iint_{\Omega} P_{1}\left(M^{\prime}, M_{0}^{\prime}\right) q\left(x_{0}, y_{0}\right) \nu\left(x_{0}, y_{0}\right) d x_{0} d y_{0}\right|^{2} d x d y \\
& \leqslant \iint_{\mathbb{R}_{2}}\left(\iint_{\Omega}\left|P_{1}\left(M^{\prime}, M_{0}^{\prime}\right)\right| q\left(x_{0}, y_{0}\right) d x_{0} d y_{0}\right. \\
& \left.\quad \times \iint_{\Omega}\left|P_{1}\left(M^{\prime}, M_{0}^{\prime}\right) q\left(x_{0}, y_{0}\right)\right|\left|\nu\left(x_{0}, y_{0}\right)\right|^{2} d x_{0} d y_{0}\right) d x d y
\end{aligned}
$$

Ввиду конструкции функции $\theta\left(\left|M_{0}^{\prime} M^{\prime}\right|\right)$ выполняются неравенства

$$
\begin{gathered}
\iint_{\Omega}\left|P_{1}\left(M^{\prime}, M_{0}^{\prime}\right) q\left(x_{0}, y_{0}\right)\right| d x_{0} d y_{0} \leqslant C_{1}, \\
\iint_{\mathbb{R}_{2}}\left|P_{1}\left(M^{\prime}, M_{0}^{\prime}\right)\right| d x d y \leqslant C_{2},
\end{gathered}
$$

где $C_{1}$ не зависит от точки $M^{\prime}(x, y)$, a $C_{2}$ - от $M_{0}^{\prime}\left(x_{0}, y_{0}\right)$.

Из (8.13) и (8.14) вытекает оценка

$$
\begin{aligned}
& \iint_{\mathbb{R}_{2}}\left|\psi_{2}(x, y)\right|^{2} d x d y \\
& \quad \leqslant C_{1} \iint_{\Omega} q\left(x_{0}, y_{0}\right)\left|\nu\left(x_{0}, y_{0}\right)\right|^{2} \cdot\left[\iint_{\mathbb{R}_{2}}\left|P_{1}\left(M^{\prime}, M_{0}^{\prime}\right)\right| d x d y\right] d x_{0} d y_{0} \\
& \quad \leqslant C_{3}\left\|\nu\left(x_{0}, y_{0}\right)\right\|_{L_{2}(\Omega)}^{2} .
\end{aligned}
$$

Из последнего неравенства следует, что оператор $\mathrm{P}_{1}(\nu)$ ограничен из $\stackrel{\circ}{H}_{1 / 2}(\Omega)$ в $H_{-1 / 2}\left(\mathbb{R}_{2}\right)$. Предложение 8.1 доказано.

Определим семейство дискретных операторов $\mathrm{B}_{1 h}$ соотношением

$$
\begin{aligned}
\pi \mathrm{B}_{1 h} u(x, y, h) & =\pi \mathrm{T}_{h} u(x, y, h)+\pi \mathrm{P}_{1 h} u(x, y, h) \\
& =\sum_{k \in \Lambda(h)} u(k) \beta_{1}(k, m)+\sum_{k \in \Lambda(h)} u(k) \beta_{2}^{\prime}(k, m),
\end{aligned}
$$

где $u(k)=\pi u(x, y, h)$, величины $\beta_{2}^{\prime}(k, m)$ определяются равенством

$$
\beta_{2}^{\prime}(k, m)=\iint_{D(k, h)} P_{1}\left(M_{0 m}^{\prime}, M_{0}^{\prime}\right) q\left(x_{0}, y_{0}\right) d x_{0} d y_{0}, \quad M_{0 m}^{\prime}\left(m_{1} h, m_{2} h\right) \in D(m, h) .
$$


ЗАмечанИЕ 8.2. Нужно отметить, что $\beta_{2}^{\prime}(k, m)=\beta_{2}(k, m)$ при $k \in \Lambda(h)$, $m \in \Lambda(h)$. Поэтому согласно предложению 8.2 семейство операторов $\mathrm{B}_{1 h} \prod_{h}^{*}$-аппроксимирует оператор $\mathrm{B}_{1}$ в области $\Omega$.

ПРЕДЛОЖЕНИЕ 8.4. Семейство операторов $\mathrm{P}_{1 h}$ равномерно ограничено из $\stackrel{\circ}{M}\left(r, h, \Omega_{1 h}\right)$ в $L_{2}\left(\Omega_{1 h}\right), r$ - любое, удовлетворяющее неравенству $0 \leqslant r \leqslant \frac{1}{2}$.

ДокАЗАТЕЛЬство. Имеют место оценки, аналогичные неравенствам (8.14):

$$
\sum_{k \in \Lambda(h)}\left|\beta_{2}^{\prime}(k, m)\right| \leqslant C_{1}, \quad \sum_{m}\left|\beta_{2}^{\prime}(k, m)\right| \leqslant C_{2},
$$

где в первом неравенстве $C_{1}$ не зависит от $m=\left(m_{1}, m_{2}\right)$; во втором неравенстве суммирование производится по всем номерам $m=\left(m_{1}, m_{2}\right), k \in \Lambda(h), D(k, h) \subset$ $\Omega_{1 h}$, константа $C_{2}$ не зависит от номера $k \in \Lambda(h)$.

Обозначим $d(m)=\pi \mathrm{P}_{1 h} u(x, y, h)$, тогда, используя (8.16) и неравенство Иенсена, получаем

$$
\begin{aligned}
h^{2} \sum_{m}|d(m)|^{2} & =h^{2} \sum_{m}\left|\sum_{k \in \Lambda(h)} u(k) \beta_{2}^{\prime}(k, m)\right|^{2} \\
& \leqslant h^{2} \sum_{m}\left(\sum_{k \in \Lambda(h)}\left|\beta_{2}^{\prime}(k, m)\right|\right)\left(\sum_{k \in \Lambda(h)}\left|\beta_{2}^{\prime}(k, m)\right| \cdot|u(k)|^{2}\right) \\
& \leqslant C_{1} h^{2} \sum_{k \in \Lambda(h)}|u(k)|^{2}\left(\sum_{m \in \Lambda(h)}\left|\beta_{2}^{\prime}(k, m)\right|\right) \leqslant C_{3}\|u(x, y, h)\|_{L_{2}}^{2} .
\end{aligned}
$$

Из последнего неравенства следует предложение 8.4.

Рассмотрим уравнение

$$
\mathrm{PB}_{1}(u)=F(x, y)
$$

где $\mathrm{P}$ - сужение оператора $\mathrm{B}_{1}$ на область $\Omega, u(x, y) \in \stackrel{\circ}{H}_{1 / 2}(\Omega), F(x, y) \in H_{-1 / 2}(\Omega)$, число $\lambda$ (см. уравнение (8.1)) таково, что оператор $\mathrm{PB}_{1}: \stackrel{\circ}{H}_{1 / 2}(\Omega) \rightarrow H_{-1 / 2}(\Omega)$ имеет ядро, состоящее из одного нулевого элемента.

Для приближенного решения уравнения (8.17) рассмотрим систему уравнений

$$
\mathrm{P}_{h} \mathrm{~B}_{1 h} u(x, y, h)=F(x, y, h),
$$

где $\mathrm{P}_{h}$ - сужение оператора $\mathrm{B}_{1 h}$ на область $\Omega_{1 h}, u(x, y, h) \in \stackrel{\circ}{M}\left(1 / 2, h, \Omega_{1 h}\right)$, $F(x, y, h) \in M\left(-1 / 2, h, \Omega_{1 h}\right)$, причем сушествуют продолжения $\widetilde{F}(x, y, h) \in$ $M(-1 / 2, h)$ функций $F(x, y, h)$ такие, что $\widetilde{F}(x, y, h) \rightarrow l F(x, y)$ по норме $H_{-1 / 2}\left(\mathbb{R}_{2}\right)$ при $h \rightarrow 0$, где $l F(x, y) \in H_{-1 / 2}\left(\mathbb{R}_{2}\right)$ - некоторое продолжение $F(x, y)$. 
ТЕОРЕМА 8.1. Пусть поверхность б удовлетворяет условиям $§ 7$, тогда решение уравнения (8.17) существует и единственно для любой функции $F(x, y) \in H_{-1 / 2}(\Omega)$. Кроме того, найдется $\delta>0$ такое, что при $0<h<\delta$ решения $u(x, y, h)$ системы (8.18) существуют, причем $u(x, y, h)$ сходятся $к$ точному решению $u(x, y)$ в $\stackrel{\circ}{H}_{r}(\Omega)$ для любого $r, 0 \leqslant r<\frac{1}{2}$, и выполняется соотношение

$$
\left\|\prod_{1 h} u(x, y)-u(x, y, h)\right\|_{\stackrel{\circ}{M\left(1 / 2, h, \Omega_{1 h}\right)}} \rightarrow 0 \quad n p u \quad h \rightarrow 0
$$

где $\prod_{1 h}$ - сужение интегрального проектора $\prod_{h}$ на $\Omega_{1 h}$.

Доказательство следует из теоремы 7.1, замечаний 8.1, 8.2, предложений 8.3, 8.4 , а также из предложений 2.3 и 2.5.

\section{Список литературы}

1. Лифанов И. К. Метод сингулярных интегральных уравнений и численньй эксперимент. М.: ТОО "Янус", 1995.

2. Лифанов И. К., Полтавский Л. Н. Пространства дробных отношений, дискретные операторы и их приложения. I // Матем. сб. 1999. Т. 190. №9. С. 41-98.

3. Эскин Г. И. Краевые задачи для эллиптических псевдодифференциальных уравнений. М.: Наука, 1973.

4. Брычков Ю. Д., Прудников А. П. Интегральные преобразования обобщенных функций. М.: Наука, 1977.

5. Дворак А. В. Невырожденность матрицы метода дискретных вихрей в задачах пространственного обтекания // Труды ВВИА им. Н.Е. Жуковского. 1986. Т. 1313. С. 441-453.

6. Lifanov I. K., Poltavskii L. N. Quadrature formulae for the Hadamard integral over a curvilinear surface // Russian J. Numer. Anal. Math. Modelling. 1998. V. 13. № 1. P. 27-44.

7. Зигмунд А. Тригонометрические ряды. Т. 1, 2. М.: Мир, 1965.

Военньй авиационньй 